\title{
Phases of Lagrangian-invariant objects in the derived category of an abelian variety
}

\author{
Alexander Polishchuk
}

\begin{abstract}
In this paper we study Lagrangian-invariant objects (LI objects for short) in the derived category $D^{b}(A)$ of coherent sheaves on an abelian variety. For every element of the complexified ample cone $D_{A}$ we construct a natural phase function on the set of LI objects, which in the case $\operatorname{dim} A=2$ gives the phases with respect to the corresponding Bridgeland stability. The construction is based on the relation between endofunctors of $D^{b}(A)$ and a certain natural central extension of groups, associated with $D_{A}$ viewed as a Hermitian symmetric space. In the case when $A$ is a power of an elliptic curve, we show that our phase function has a natural interpretation in terms of the Fukaya category of the mirror dual abelian variety. As a by-product of our study of LI objects we show that the Bridgeland component of the stability space of an abelian surface contains all full stabilities.
\end{abstract}

\section{Contents}

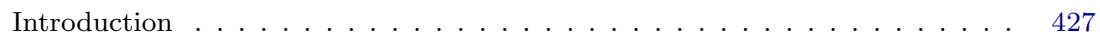

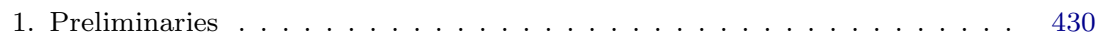

2. LI functors and central extensions . . . . . . . . . . . . . . . . . . . . . . . . . . . . . . . . . . . . . . . . .

3. Action on stability spaces . . . . . . . . . . . . . . . . . . . . . 457

4. Quasistandard $t$-structures and Fourier-Mukai partners . . . . . . . . . . 475

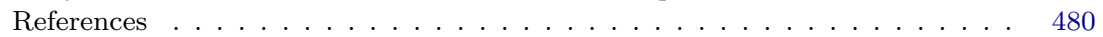

\section{Introduction}

The notion of stability condition on triangulated categories, introduced by Bridgeland in [6], axiomatizes the notion of stability of branes coming from the study of deformations of superconformal field theories (see [8]). The hope is that the space of stability conditions on a Calabi-Yau threefold is related to the moduli spaces of complex structures on a mirror dual manifold. At present we have examples of Bridgeland stabilities on $D^{b}(X)$ for any surface $X$. A program for constructing such examples for Calabi-Yau threefolds was proposed in [2]. It was implemented in [16] and [17] for some three-dimensional abelian varieties.

The goal of this paper is to test the existence of a stability condition on $D^{b}(A)$ for any abelian variety $A$ by looking at certain special objects in $D^{b}(A)$.

Kyoto Journal of Mathematics, Vol. 54, No. 2 (2014), 427-482

DOI 10.1215/21562261-2642449, (C) 2014 by Kyoto University

Received April 10, 2012. Revised October 5, 2012. Accepted March 13, 2013.

2010 Mathematics Subject Classification: Primary 14F05; Secondary 14K05, 53D37.

Author's work supported in part by a National Science Foundation grant. 
More precisely, for an element $\omega=i \alpha+\beta \in D_{A} \subset \mathrm{NS}(A) \otimes \mathbb{C}$ in the complexified ample cone (defined by the condition that $\alpha$ is ample), one expects to have a stability condition on $D^{b}(A)$ with the central charge

$$
Z(F)=\int_{A} \exp (-\omega) \cdot \operatorname{ch}(F)
$$

where $F \in D^{b}(A)$. The starting point of this work is the observation that there are certain objects in $D^{b}(A)$ that are automatically semistable with respect to any nice stability condition (see Proposition 3.1.4). Namely, these are Lagrangianinvariant objects (LI objects for short) defined in [31] (see also Definition 2.1.1). The simplest examples are the structure sheaves of points $\mathcal{O}_{x}$. To get other examples one can consider images of $\mathcal{O}_{x}$ under autoequivalences of $D^{b}(A)$, but in general these do not exhaust all LI objects (see Remark 4.2 .2 and Proposition 4.2.3). Thus, a stability condition should give a phase for any LI object $F$, that is, a lifting of $\operatorname{Arg} Z(F) \in \mathbb{R} / 2 \pi \mathbb{Z}$ to $\mathbb{R}$. Furthermore, a nonzero morphism $F_{1} \rightarrow F_{2}$ can exist only if the phase of $F_{1}$ does not exceed the phase of $F_{2}$. The main result of this paper is the construction of such a phase function associated with each $\omega \in D_{A}$. We also verify some properties of this function that one expects from the theory of stability conditions (see Theorem 3.3.2).

The major role in our construction is played by the action of a certain group on the set $\overline{\mathrm{SH}}^{\mathrm{LI}} / \mathbb{N}^{*}$ of classes of LI objects modulo the equivalence relation generated by translations and tensoring with line bundles in $\operatorname{Pic}^{0}(A)$ and with vector spaces. This group, which we denote by $\widehat{\mathbf{U}(\mathbb{Q})}$, is a central extension by $\mathbb{Z}$ of the group of $\mathbb{Q}$-points of an algebraic group $\mathbf{U}=\mathbf{U}_{X_{A}}$ defined as automorphisms of the abelian variety $X_{A}=A \times \hat{A}$ compatible with the skew-symmetric autoduality of $X_{A}$. The preimage of the subgroup of $\mathbb{Z}$-points in $\widehat{\mathbf{U}(\mathbb{Q})}$ is closely related to the group of autoequivalences of $D^{b}(A)$ (see [21], [23], [25]). The main idea that brings the Siegel domain $D_{A}$ into the picture is that the above central extension has a natural interpretation in terms of the action of $\mathbf{U}(\mathbb{Q})$ on $D_{A}$ (see Theorem 2.3.2). This allows us to parameterize the set $\overline{\mathrm{SH}}^{\mathrm{LI}} / \mathbb{N}^{*}$ of classes of LI objects by points of a natural $\mathbb{Z}$-covering of the set of $\mathbb{Q}$-points of a certain homogeneous algebraic variety $\mathbf{L G}=\mathbf{L G}_{A}$ for the group $\mathbf{U}$ (the points $\mathbf{L} \mathbf{G}(\mathbb{Q})$ are in bijection with Lagrangian abelian subvarieties in $A \times \hat{A}$ ), and the phase function appears naturally in this context.

If $\operatorname{dim} A=2$, then the stability condition corresponding to $\omega$ was constructed by Bridgeland in [7], and we check that our phases for LI objects match the ones coming from this stability condition (see Section 3.4).

In the case when $A=E^{n}$, where $E$ is an elliptic curve without complex multiplication, we give a mirror-symmetric interpretation of our picture in terms of the Fukaya category of the mirror dual abelian variety (following the recipe of [12]). We show that the central charge on LI objects in $D^{b}(A)$ defined using $\omega \in D_{A}$ matches the integral of the holomorphic volume form over the corresponding Lagrangian tori and, hence, that LI objects in $D^{b}(A)$ give rise to graded Lagrangians on the mirror dual side (see Section 3.5). 
We also observe that the set $\widetilde{\mathbf{L G}(\mathbb{Q})}$ parameterizing classes of LI objects also parameterizes a certain natural collection of $t$-structures on $D^{b}(A)$, generalizing the ones obtained from the standard $t$-structure by applying autoequivalences. (We call them quasistandard.) We conjecture that there is also a natural $t$-structure associated with every point of $\widehat{\mathbf{L G}(\mathbb{R})}$ whose heart is equivalent to the category of holomorphic bundles on the corresponding noncommutative torus (see [4], [28], [32]).

Another by-product of our study is a refinement of the results of [21] and [12] on the action of autoequivalences of $D^{b}(A)$ on numerical classes of objects. Namely, we construct a natural double covering Spin $\rightarrow \mathbf{U}$ of algebraic groups over $\mathbb{Q}$ and an algebraic representation of Spin on the vector space associated with the numerical Grothendieck group of $A$ such that the action of elements projecting to $\mathbf{U}(\mathbb{Q})$ is induced by endofunctors of $D^{b}(A)$ (see Theorem 2.5.3).

The paper is organized as follows. Section 1 contains some auxiliary results not involving derived categories. In particular, we give an interpretation of the index of a nondegenerate line bundle on an abelian variety in terms of the function $\operatorname{Arg} \chi$ on the complexified ample cone (see Theorem 1.2.1). We also prove some useful results about the group $\mathbf{U}$ and the variety of Lagrangian subvarieties $\mathbf{L G}$ in $A \times \hat{A}$. In Section 2 we study the central extension $\widehat{\mathbf{U}(\mathbb{Q})} \rightarrow \mathbf{U}(\mathbb{Q})$ coming from a natural 1-cocycle with values in $\mathcal{O}^{*}\left(D_{A}\right)$ and its action on LI objects in $D^{b}(A)$ and their numerical classes. In Section 3 we parameterize LI objects (up to a certain equivalence) by points of a natural $\mathbb{Z}$-covering $\widetilde{\mathbf{L G}(\mathbb{Q})} \rightarrow \mathbf{L G}(\mathbb{Q})$ equipped with an action of $\widehat{\mathbf{U}(\mathbb{Q})}$, and we construct a family of phase functions on $\widetilde{\mathbf{L G}(\mathbb{Q})}$ parameterized by $D_{A} \times \mathbb{C}$, equivariantly with respect to $\widetilde{\mathbf{U}(\mathbb{Q})}$. We also study the connection with Bridgeland stability conditions on abelian surfaces (see Theorem 3.4.3) and with mirror symmetry (see Section 3.5). Also, as a byproduct of our study of LI objects we show that any full stability on an abelian surface $A$ belongs to the Bridgeland component of the stability space $\operatorname{Stab}(A)$ (see Proposition 3.4.4). In Section 4 we construct a family of $t$-structures on $D^{b}(A)$ parameterized by $\widehat{\mathbf{L G}(\mathbb{Q})}$ and study a relation between $\mathbf{L G}(\mathbb{Q}) / \mathbf{U}(\mathbb{Q})$ and the Fourier-Mukai partners of $A$ (see Section 4.2).

\section{NOTATIONS AND CONVENTIONS}

We work over a fixed algebraically closed field $k$. We say that an object $F$ of a $k$ linear category $\mathcal{C}$ is endosimple if $\operatorname{Hom}_{\mathcal{C}}(F, F)=k$. For a scheme $X$ we denote by $D^{b}(X)$ the bounded derived category of coherent sheaves on $X$. We say that an object $F \in D^{b}(X)$ is cohomologically pure if there exists a coherent sheaf $H$ such that $F \simeq H[n]$ for $n \in \mathbb{Z}$. We denote by $\mathcal{A b}_{\mathbb{Q}}$ the category of abelian varieties up to an isogeny (i.e., the localization of the category of abelian varieties over $k$ with respect to the class of isogenies). When we want to consider the $F$-vector space associated with a $\mathbb{Z}$-lattice $M$, where $F=\mathbb{Q}, \mathbb{R}$, or $\mathbb{C}$, as an algebraic variety over $F$, we denote it by $M_{F}$. 


\section{Preliminaries}

Throughout this paper $A$ denotes an abelian variety over $k$.

\subsection{Degree, trace, and Euler bilinear form}

Recall that for $f \in \operatorname{End}(A)$ one has

$$
\operatorname{deg}(f)=\operatorname{det} T_{l}(f),
$$

where $T_{l}(f)$ is the representation of $f$ on the Tate module $T_{l}(A)$ for $l \neq \operatorname{char}(k)$ (see [22, Chapter 19, Theorem 4]). Thus, extending deg to a polynomial function

$$
\operatorname{deg}: \operatorname{End}(A) \otimes \mathbb{Q} \rightarrow \mathbb{Q},
$$

homogeneous of degree $2 g$, we have

$$
\operatorname{deg}(1+t f)=1+t \cdot \operatorname{Tr}(f)+O\left(t^{2}\right),
$$

where $\operatorname{Tr}(f)$ is given by the trace of the action of $f$ on $T_{l}(A) \otimes_{\mathbb{Z}_{l}} \mathbb{Q}_{l}$. Furthermore, $f \mapsto \operatorname{Tr}\left(f \cdot f^{\prime}\right)$ is a positive definite quadratic form on $\operatorname{End}(A) \otimes \mathbb{Q}$, where $f \mapsto f^{\prime}$ is the Rosati involution associated with a polarization of $A$ (see [22, Chapter 21, Theorem 1]).

Let us fix a polarization on $A$, and we denote by $\operatorname{End}(A)^{+} \otimes \mathbb{Q} \subset \operatorname{End}(A) \otimes$ $\mathbb{Q}$ the subspace of elements invariant with respect to the corresponding Rosati involution. Note that the quadratic form $\operatorname{Tr}\left(f^{2}\right)$ on $\operatorname{End}(A)^{+} \otimes \mathbb{Q}$ is positive definite.

PROPOSITION 1.1.1

An element $f \in \operatorname{End}(A) \otimes \mathbb{C}$ is determined by the polynomial function

$$
\operatorname{End}(A) \otimes \mathbb{C} \rightarrow \mathbb{C}: x \mapsto \operatorname{deg}(f-x) .
$$

Furthermore, if $f$ is invariant with respect to the Rosati involution, then it is determined by the restriction of the above function to $\operatorname{End}(A)^{+} \otimes \mathbb{C}$.

Proof

We have to check that if $\operatorname{deg}\left(f_{1}-x\right)=\operatorname{deg}\left(f_{2}-x\right)$ for all $x \in \operatorname{End}(A)$, then $f_{1}=f_{2}$. Adding to $f_{1}$ and $f_{2}$ the same element of $\operatorname{End}(A) \otimes \mathbb{C}$, we can assume that $f_{1}$ and $f_{2}$ are invertible in $\operatorname{End}(A) \otimes \mathbb{C}$. Observe also that $\operatorname{deg}\left(f_{1}\right)=\operatorname{deg}\left(f_{2}\right)$. (This follows by substituting $x=0$.) Thus, we obtain

$\operatorname{deg}\left(1-x f_{1}^{-1}\right)=\operatorname{deg}\left(f_{1}-x\right) \operatorname{deg}\left(f_{1}\right)^{-1}=\operatorname{deg}\left(f_{2}-x\right) \operatorname{deg}\left(f_{2}\right)^{-1}=\operatorname{deg}\left(1-x f_{2}^{-1}\right)$.

Considering the linear terms in $x$ we derive

$$
\operatorname{Tr}\left(x f_{1}^{-1}\right)=\operatorname{Tr}\left(x f_{2}^{-1}\right) .
$$

The nondegeneracy of the form $\operatorname{Tr}(f g)$ implies that $f_{1}^{-1}=f_{2}^{-1}$.

To prove the second statement, we repeat the above argument, letting $x$ vary only in $\operatorname{End}(A)^{+} \otimes \mathbb{C}$. 
We always use the standard identification

$$
\mathrm{NS}(A) \otimes \mathbb{Q} \stackrel{\sim}{\longrightarrow} \operatorname{Hom}(A, \hat{A})^{+} \otimes \mathbb{Q}: L \mapsto \phi_{L},
$$

where $\operatorname{Hom}(A, \hat{A})^{+} \otimes \mathbb{Q} \subset \operatorname{Hom}(A, \hat{A}) \otimes \mathbb{Q}$ consists of self-dual homomorphisms. The Euler characteristic defines a polynomial function $\chi: \operatorname{NS}(A) \otimes \mathbb{C} \rightarrow \mathbb{C}$ of degree $g=\operatorname{dim} A$, which we also view as a function on $\operatorname{Hom}(A, \hat{A})^{+} \otimes \mathbb{C}$. One has $\chi^{2}=\operatorname{deg}($ see $[22$, Chapter 16]).

Recall that the Grothendieck group $K_{0}(A)$ carries the Euler bilinear form

$$
\chi([E],[F]):=\sum_{i}(-1)^{i} \operatorname{dim} \operatorname{Hom}^{i}(E, F),
$$

where $E, F \in D^{b}(A)$. We denote by $\mathcal{N}(A)$ the numerical Grothendieck group, that is, the quotient of $K_{0}(A)$ by the kernel of this form; $\mathcal{N}(A)$ is a free abelian group of finite rank (see [9, Example 19.1.4]). Associating with a line bundle $L$ its class $[L]$ in $\mathcal{N}(A)$ defines a polynomial map between free abelian groups of finite rank

$$
\ell: \mathrm{NS}(A) \rightarrow \mathcal{N}(A) .
$$

Therefore, we have the induced polynomial morphism between the corresponding $\mathbb{Q}$-vector spaces

$$
\ell: \mathrm{NS}(A)_{\mathbb{Q}} \rightarrow \mathcal{N}(A)_{\mathbb{Q}}
$$

\section{COROLLARY 1.1.2}

An element $\phi \in \mathrm{NS}(A) \otimes \mathbb{C}$ is determined by the corresponding polynomial function

$$
\mathrm{NS}(A) \rightarrow \mathbb{C}: x \mapsto \chi(\ell(\phi), \ell(x))
$$

Proof

Since $\operatorname{NS}(A)$ is Zariski-dense in $\operatorname{NS}(A)_{\mathbb{C}}$, it is enough to prove the similar statement with the polynomial function $\chi(\ell(\phi), \ell(\cdot))$ on $\operatorname{NS}(A) \otimes \mathbb{C}$. Note that

$$
\chi(\ell(\phi), \ell(x))^{2}=\chi(\ell(x-\phi))^{2}=\operatorname{deg}(x-\phi),
$$

where we view $x$ and $\phi$ as elements of $\operatorname{Hom}(A, \hat{A})^{+} \otimes \mathbb{C}$. Let $\phi_{0}: A \rightarrow \hat{A}$ be a polarization. Then the map $x \mapsto \phi_{0}^{-1} \circ x$ gives an isomorphism $\operatorname{Hom}(A, \hat{A})^{+} \otimes \mathbb{Q} \simeq$ $\operatorname{End}(A)^{+} \otimes \mathbb{Q}$ (and the corresponding isomorphism of $\mathbb{C}$-vector spaces). Furthermore, this isomorphism rescales deg by the constant $\operatorname{deg}\left(\phi_{0}\right)$. It remains to apply Proposition 1.1.1.

\section{REMARK 1.1.3}

When the ground field is $\mathbb{C}$ we can identify $\mathcal{N}(A) \otimes \mathbb{Q}$ with the subspace of algebraic cycles in $H^{*}(A, \mathbb{Q})$ via the Chern character and $\mathrm{NS}(A) \otimes \mathbb{Q}$ with algebraic cycles in $H^{2}(A, \mathbb{Q})$. Then $\ell$ is induced by the exponential map $H^{2}(A, \mathbb{Q}) \rightarrow$ $H^{*}(A, \mathbb{Q})$. 


\subsection{Characterization of the index of a line bundle}

Recall that if $L$ is a nondegenerate line bundle on $A$ (i.e., the corresponding map $\phi_{L}: A \rightarrow \hat{A}$ is an isogeny), then its index $i(L)$ is defined by the condition $H^{i}(A, L)=0$ for $i \neq i(L)$. We will use the following recipe for computing $i(L)$ : it is the number of positive roots of the polynomial $P(n)=\chi\left(L \otimes L_{0}^{n}\right)$, where $L_{0}$ is an ample line bundle on $A$ (see [22, Chapter 16]). The index function $i(\cdot)$ extends uniquely to a $\mathbb{Q}_{>0}$-invariant function on $\mathrm{NS}(A)_{\mathbb{Q}}$.

Let $D_{A} \subset \mathrm{NS}(A) \otimes \mathbb{C}$ be the complexified ample cone. Note that the function deg and hence $\chi$ do not vanish on $D_{A}$ (see [12, Lemma A.3]). Since $D_{A}$ is simply connected, there is a unique continuous branch of the $\operatorname{argument} \operatorname{Arg}(\chi(x))$ on $D_{A}$ such that, for $x=i H$, where $H$ is an ample class (an element of the ample cone), we have $\operatorname{Arg}(\chi(i H))=g \pi / 2$, where $g=\operatorname{dim} A$. It is easy to see that this branch does not depend on a choice of $H$. Then for a class $x \in \operatorname{NS}(A) \otimes \mathbb{R}$ with $\chi(x) \neq 0$ we can define by continuity the argument $\operatorname{Arg}(\chi(x))$; that is, we set

$$
\operatorname{Arg}(\chi(x))=\lim _{t \rightarrow 0+} \operatorname{Arg}(\chi(x+i t H)),
$$

where $H$ is an ample class. Note that, since $\chi(x)$ is real, the number $\operatorname{Arg}(\chi(x)) / \pi$ is an integer.

\section{THEOREM 1.2.1}

For the continuous branch of $\operatorname{Arg}(\chi(\cdot))$ on $D_{A}$, satisfying $\operatorname{Arg}(\chi(i H))=g \pi / 2$ (where $H$ is ample), one has

$$
\operatorname{Arg}(\chi(x))=i(x) \pi
$$

for every $x \in \mathrm{NS}(A) \otimes \mathbb{Q}$ with $\chi(x) \neq 0$.

Proof

First, let us consider the case when $x$ is in the ample cone. For $z \in \mathbb{C}$ we have $\chi(z x)=z^{g} \cdot \chi(x)$. Thus, varying $z$ on a unit circle from 1 to $i$ we obtain

$$
\operatorname{Arg}(\chi(i x))=\operatorname{Arg}(\chi(x))+\frac{g \pi}{2} .
$$

Since $\operatorname{Arg}(\chi(i x))=g \pi / 2$, we obtain that $\operatorname{Arg}(\chi(x))=0$. Next, assume that $x \in$ $\mathrm{NS}(A) \subset \mathrm{NS}(A) \otimes \mathbb{Q}$. Then for any ample class $H$ the polynomial

$$
P(t)=\chi(x+t H)
$$

has $i(x)$ positive roots, counted with multiplicity (see [22, Chapter 16]). Let $0<t_{1}<\cdots<t_{r}$ be all the positive roots of $P(t)$. For $t \gg 0$ the class $x+t H$ is ample, and so $\operatorname{Arg} \chi(x+t H)=0$. Now we are going to decrease $t$ until it reaches zero and observe the change of $\operatorname{Arg}(P(t))=\operatorname{Arg}(\chi(x+t H))$. Note that it can change only when $t$ passes one of the roots $t_{j}$. If $t_{j}$ is a root of $P(t)$ of multiplicity $m_{j}$, then for sufficiently small $\epsilon>0$ one has

$$
\operatorname{Arg}\left(P\left(t_{j}-\epsilon\right)\right)=\operatorname{Arg}\left(P\left(t_{j}+\epsilon\right)\right)+m_{j} \pi .
$$


Adding up the changes we get that

$$
\operatorname{Arg}(\chi(x))=\operatorname{Arg}(P(0))=i(x) \pi
$$

Since $i(x)$ does not change upon rescaling by a positive rational number, the assertion for any $x \in \mathrm{NS}(A) \otimes \mathbb{Q}$ follows.

\section{COROLLARY 1.2.2}

For the branch of $\operatorname{Arg}(\operatorname{deg}(\cdot))$ on $D_{A}$ normalized by $\operatorname{Arg}(\operatorname{deg}(i H))=g \pi$ one has

$$
\operatorname{Arg}(\operatorname{deg}(x))=i(x) \cdot 2 \pi
$$

for any $x \in \mathrm{NS}(A) \otimes \mathbb{Q}$ such that $\operatorname{deg}(x) \neq 0$.

We will also need some information on the restriction of $\operatorname{Arg}(\chi(\cdot))$ to lines of the form $i H+\mathbb{R} x \subset D_{A}$.

\section{LEMMA 1.2.3}

(i) For any ample class $H \in \mathrm{NS}(A) \otimes \mathbb{Q}$ and any $x \in \mathrm{NS}^{0}(A, \mathbb{Q})$ let us choose any continuous branch of $t \mapsto \operatorname{Arg}(\chi(i H+t x))$, where $t \in \mathbb{R}$. Then for $0 \leq t_{1}<t_{2}$ one has

$$
\begin{aligned}
\operatorname{Arg}\left(\chi\left(i H+t_{1} x\right)\right)-(g-i(x)) \frac{\pi}{2} & <\operatorname{Arg}\left(\chi\left(i H+t_{2} x\right)\right) \\
& <\operatorname{Arg}\left(\chi\left(i H+t_{1} x\right)\right)+i(x) \frac{\pi}{2} .
\end{aligned}
$$

(ii) For any continuous branch of $\operatorname{Arg}(\operatorname{deg}(\cdot))$ on $D_{A}$ one has

$$
\operatorname{Arg}(\operatorname{deg}(\omega)) \leq \operatorname{Arg}(\operatorname{deg}(i H))+g \pi
$$

for any $\omega \in D_{A}$, where $H$ is an ample class.

\section{Proof}

(i) Indeed, the polynomial

$$
P(t)=\chi(i H+t x)=i^{g} \chi\left(H+\frac{t}{i} x\right)
$$

has all roots purely imaginary, and exactly $i(x)$ of them are in the upper halfplane, counted with multiplicity (see [22, Chapter 16]). Let us write $P(t)=c \cdot(t-$ $\left.z_{1}\right) \cdot \ldots \cdot\left(t-z_{g}\right)$. Since $P(t) \neq 0$ for all $t \in \mathbb{R}$, we can choose for every $j=1, \ldots, g$ a continuous branch of $t \mapsto \operatorname{Arg}\left(t-z_{j}\right)$ along the real line and use the branch

$$
\operatorname{Arg} P(t)=\operatorname{Arg}(c)+\operatorname{Arg}\left(t-z_{1}\right)+\cdots+\operatorname{Arg}\left(t-z_{g}\right) .
$$

Suppose the roots $z_{1}, \ldots, z_{i(x)}$ are in the upper half-plane while the $z_{j}$ 's for $j>i(x)$ are in the lower half-plane. Then for each $j>i(x)$ the function $t \mapsto$ $\operatorname{Arg}\left(t-z_{j}\right)$ is strictly decreasing, and we have

$$
\operatorname{Arg}\left(t_{1}-z_{j}\right)-\frac{\pi}{2}<\operatorname{Arg}\left(t_{2}-z_{j}\right)<\operatorname{Arg}\left(t_{1}-z_{j}\right) .
$$


On the other hand, for $j \leq i(x)$ we have

$$
\operatorname{Arg}\left(t_{1}-z_{j}\right)<\operatorname{Arg}\left(t_{2}-z_{j}\right)<\operatorname{Arg}\left(t_{1}-z_{j}\right)+\frac{\pi}{2} .
$$

Summing up over all the roots gives (1.2.1).

(ii) Applying (1.2.1) to $t_{1}=0$ and $t_{2}=1$ we get

$$
\operatorname{Arg}(\chi(i H+x)) \leq \operatorname{Arg}(\chi(i H))+i(x) \frac{\pi}{2} \leq \operatorname{Arg}(\chi(i H))+g \frac{\pi}{2} .
$$

Since deg $=\chi^{2}$ on NS, we get the required inequality for points in $D_{A}$ with rational real and imaginary parts. The general case follows by continuity.

\subsection{The group $\mathbf{U}_{A \times \hat{A}}$}

Recall (see [12], [21], [23], [24]) that with every abelian variety $A$ one can associate an algebraic group $\mathbf{U}=\mathbf{U}_{X_{A}}$ over $\mathbb{Q}$, where $X_{A}:=A \times \hat{A}$, as follows. For every $F \subset \mathbb{Q}$ we define the group of $F$-points $\mathbf{U}(F)$ as a subgroup of invertible elements of the algebra $\operatorname{End}\left(X_{A}\right) \otimes F$ consisting of $g=\left(\begin{array}{ll}a & b \\ c & d\end{array}\right) \in \operatorname{End}\left(X_{A}\right) \otimes F$ with $a \in$ $\operatorname{Hom}(A, A) \otimes F, b \in \operatorname{Hom}(\hat{A}, A) \otimes F$, and so forth, such that

$$
g^{-1}=\left(\begin{array}{cc}
\hat{d} & -\hat{b} \\
-\hat{c} & \hat{a}
\end{array}\right) \in \operatorname{End}(A \times \hat{A}) \otimes F .
$$

The arithmetic subgroup

$$
\mathbf{U}(\mathbb{Z}):=\mathbf{U}(\mathbb{Q}) \cap \operatorname{End}(A \times \hat{A})
$$

is closely related to the group of autoequivalences of $D^{b}(A)$ (see [23]). When we view the matrix element $b$ above as a function on $\mathbf{U}(F)$ we denote it by $b(g)$.

Our point of view is to consider $X_{A}$ as a "symplectic object" in the category of abelian varieties using the skew-symmetric self-duality $\eta_{A}: X_{A} \widetilde{\rightarrow} \hat{X}_{A}$ associated with the biextension $p_{14}^{*} \mathcal{P} \otimes p_{23}^{*} \mathcal{P}^{-1}$ of $X_{A} \times X_{A}$ (see [25], [31]). Then elements of $\mathbf{U}(\mathbb{Z})$ are precisely symplectic automorphisms of $X_{A}$, that is, automorphisms compatible with $\eta_{A}$. The development of this point of view in [31] was to view elements of $\mathbf{U}(\mathbb{Q})$ as Lagrangian correspondences from $X_{A}$ to itself, which allowed us to define endofunctors of $D^{b}(A)$ associated with elements of $\mathbf{U}(\mathbb{Q})$ (see [31, Section 3] and Section 2.1 below).

Note that we have the algebraic subgroup $\mathbf{T} \simeq\left(\operatorname{End}(A)_{\mathbb{Q}}\right)^{*} \subset \mathbf{U}$ consisting of diagonal matrices of the form

$$
\left(\begin{array}{cc}
a^{-1} & 0 \\
0 & \hat{a}
\end{array}\right)
$$

The following facts about the group $\mathbf{U}$ follow easily from Albert's classification of the endomorphism algebras of simple abelian varieties (see [12], [24]).

\section{LEMMA 1.3.1}

(i) Let us fix a polarization $\phi: A \rightarrow \hat{A}$, and let $\mathbf{Z} \subset \mathbf{T}$ be the algebraic subgroup corresponding to $a \in\left(\operatorname{End}(A)_{\mathbb{Q}}\right)^{*}$ such that a lies in the center of $\operatorname{End}(A)_{\mathbb{Q}}$ and $a^{-1}=\phi^{-1} \hat{a} \phi$. Then the group $\mathbf{U}$ is an almost direct product of the semisimple commutant subgroup $S \mathbf{U}$ and of $\mathbf{Z}$. 
(ii) The algebraic group $\mathbf{U}$ is connected, and the Lie group $\mathbf{U}(\mathbb{R})$ is connected (with respect to the classical topology).

We denote by $\mathbf{U}^{0} \subset \mathbf{U}$ the Zariski-open subset given by the inequality $\operatorname{deg}(b(g)) \neq 0$. Note that for any $g \in \mathbf{U}^{0}(\mathbb{R})$ we have $\operatorname{deg}(b(g))>0$ (since the function $\operatorname{deg}$ is nonnegative on $\operatorname{Hom}(A, \hat{A}) \otimes \mathbb{R})$.

The following condition on a subset of a group was introduced in [34, Section IV.42]. (The term is due to D. Kazhdan.)

\section{DEFINITION 1.3.2}

Let $G$ be a group. A subset $B \subset G$ is called big if for any $g_{1}, g_{2}, g_{3} \in G$ one has

$$
B^{-1} \cap B g_{1} \cap B g_{2} \cap B g_{3} \neq \emptyset \text {. }
$$

This notion is useful because of the following result. (Part (i) is due to Weil, and part (ii) is a more precise version of [26, Lemma 4.2].)

LEMMA 1.3.3

(i) Let $B \subset G$ be a big subset. Then $G$ is isomorphic to the abstract group generated by elements $[b]$ for $b \in B$ subject to the relations $\left[b_{1}\right]\left[b_{2}\right]=\left[b_{1} b_{2}\right]$ whenever $b_{1} b_{2} \in B$.

(ii) Let $Z$ be an abelian group (with the trivial $G$-action). Let $c, c^{\prime}: G \times G \rightarrow$ $Z$ be a pair of 2-cocycles such that

$$
c\left(b_{1}, b_{2}\right)=c^{\prime}\left(b_{1}, b_{2}\right)
$$

for any $b_{1}, b_{2} \in B$ with $b_{1} b_{2} \in B$. Let $p: G_{c} \rightarrow G$ (resp., $p^{\prime}: G_{c^{\prime}} \rightarrow G$ ) be the extension of $G$ by $Z$ associated with $c$ (resp., $c^{\prime}$ ), and let $\sigma: G \rightarrow G_{c}$ (resp., $\left.\sigma^{\prime}: G \rightarrow G_{c^{\prime}}\right)$ be the natural set-theoretic sections. Then there is a unique isomorphism of extensions $i: G_{c} \rightarrow G_{c^{\prime}}$ such that $i(\sigma(b))=\sigma^{\prime}(b)$ (and identical on $Z$ ).

\section{Proof}

(i) This is $[34$, IV.42, Lemma 6].

(ii) Note that the subset $p^{-1}(B) \subset G_{c}$ (resp., $\left.\left(p^{\prime}\right)^{-1}(B) \subset G_{c^{\prime}}\right)$ is big. Thus, we can define a homomorphism $G_{c} \rightarrow G_{c^{\prime}}$ by requiring that it sends $z \sigma(b)$ to $z \sigma^{\prime}(b)$ for $b \in B$, provided we check the compatibility with the relations

$$
\begin{aligned}
\sigma\left(b_{1}\right) \sigma\left(b_{2}\right) & =c\left(b_{1}, b_{2}\right) \sigma\left(b_{1} b_{2}\right), \\
\sigma^{\prime}\left(b_{1}\right) \sigma^{\prime}\left(b_{2}\right) & =c^{\prime}\left(b_{1}, b_{2}\right) \sigma^{\prime}\left(b_{1} b_{2}\right),
\end{aligned}
$$

whenever $b_{1}, b_{2}, b_{1} b_{2} \in B$. But this boils down to the equality $c\left(b_{1}, b_{2}\right)=c^{\prime}\left(b_{1}, b_{2}\right)$.

Next, we will show that the subset $\mathbf{U}^{0}(\mathbb{Q}) \subset \mathbf{U}(\mathbb{Q})\left(\right.$ resp., $\mathbf{U}^{0}(\mathbb{R}) \subset \mathbf{U}(\mathbb{R})$ ) is big. Note that the subset $\mathbf{U}^{0}(\mathbb{Q}) \cap \mathbf{U}(\mathbb{Z})$ in the arithmetic group $\mathbf{U}(\mathbb{Z})$ is also big (see Remark 1.4.2). 
LEMMA 1.3.4

For any field extension $\mathbb{Q} \subset F$ the set $\mathbf{U}(F)$ is Zariski-dense in $\mathbf{U}$. Hence, the subset $\mathbf{U}^{0}(F) \subset \mathbf{U}(F)$ is big in $\mathbf{U}(F)$.

Proof

Since $\mathbf{U}$ is connected, the density of $\mathbf{U}(F)$ follows from [5, Corollary 18.3]. Thus, for any $g_{1}, g_{2}, g_{3} \in \mathbf{U}(F)$ the intersection $\mathbf{U}^{0} \cap \mathbf{U}^{0} g_{1} \cap \mathbf{U}^{0} g_{2} \cap \mathbf{U}^{0} g_{3}$ contains a point of $\mathbf{U}(F)$.

The group $\mathbf{U}$ has two natural parabolic subgroups: $\mathbf{P}^{+}$is the intersection of $\mathbf{U}$ with the subgroup of upper-triangular $2 \times 2$ matrices in $\operatorname{End}(A \times \hat{A})_{\mathbb{Q}}$, and $\mathbf{P}^{-}$is the intersection with the subgroup of lower-triangular matrices. We also denote by $\mathbf{N}^{+} \subset \mathbf{P}^{+}$(resp., $\mathbf{N}^{-} \subset \mathbf{P}^{-}$) the subgroup of strictly upper-triangular (resp., strictly lower-triangular) matrices. Note that both $\mathbf{N}^{+}$and $\mathbf{N}^{-}$are isomorphic to $\mathrm{NS}(A)_{\mathbb{Q}}$.

\section{LEMMA 1.3.5}

Any normal subgroup of $\mathbf{U}(\mathbb{Q})$ containing $\mathbf{P}^{-}(\mathbb{Q})$ is the entire $\mathbf{U}(\mathbb{Q})$.

Proof

Since $\mathbf{P}^{+}(\mathbb{Q})$ is conjugate to $\mathbf{P}^{-}(\mathbb{Q})$ by an element

$$
w_{\phi}=\left(\begin{array}{cc}
0 & \phi^{-1} \\
-\phi & 0
\end{array}\right)
$$

where $\phi: A \rightarrow \hat{A}$ is a polarization, it is enough to check that $\mathbf{U}(\mathbb{Q})$ is generated by the subgroups $\mathbf{P}^{+}(\mathbb{Q})$ and $\mathbf{P}^{-}(\mathbb{Q})$. We can write any $g=\left(\begin{array}{ll}a & b \\ c & d\end{array}\right) \in \mathbf{U}(\mathbb{Q})$ with invertible $a$ as

$$
g=\left(\begin{array}{cc}
1 & 0 \\
c a^{-1} & 1
\end{array}\right) \cdot\left(\begin{array}{cc}
a & 0 \\
0 & \hat{a}^{-1}
\end{array}\right) \cdot\left(\begin{array}{cc}
1 & a^{-1} b \\
0 & 1
\end{array}\right)
$$

Finally, any element of $\mathbf{U}^{0}(\mathbb{Q})$ has form $g w_{\phi}$ with $g$ as above. Thus, the statement follows from Lemma 1.3.4.

\subsection{Action of $U(\mathbb{Q})$ on Lagrangian subvarieties}

Recall that an abelian subvariety $L \subset X_{A}=A \times \hat{A}$ is isotropic if the composition

$$
L \rightarrow X_{A} \stackrel{\eta_{A}}{\longrightarrow} \hat{X}_{A} \rightarrow \hat{L}
$$

is zero, where $\eta_{A}$ is the standard skew-symmetric self-duality. If in addition $\operatorname{dim} L=\operatorname{dim} A$, then $L$ is called Lagrangian (for other equivalent definitions see [31, Section 2.2]). In this case $\eta_{A}$ induces an isomorphism $X_{A} / L \simeq \hat{L}$.

To enumerate all Lagrangian abelian subvarieties in $X_{A}$ it is convenient to work in the semisimple category $\mathcal{A}_{\mathbb{Q}}$ of abelian varieties up to isogeny. Note that abelian subvarieties of $X_{A}$ are in natural bijection with subobjects of $X_{A}$ in the category $\mathcal{A b}_{\mathbb{Q}}$. Thus, we can use a similar notion of a Lagrangian subvariety in $\mathcal{A} \mathrm{b}_{\mathbb{Q}}$. Now if $L \subset X_{A}$ is Lagrangian, then we have an isomorphism $X_{A} \simeq L \oplus \hat{L}$ in 
$\mathcal{A} \mathrm{b}_{\mathbb{Q}}$, which implies that $L$ is isomorphic to $A$ in $\mathcal{A} \mathrm{b}_{\mathbb{Q}}$. Thus, we can describe a Lagrangian subvariety (in the category $\mathcal{A} \mathrm{b}_{\mathbb{Q}}$ ) as an image of a morphism $A \rightarrow X_{A}$, that is, by a pair $(x, y)$, where $x \in \operatorname{End}(A) \otimes \mathbb{Q}, y \in \operatorname{Hom}(A, \hat{A}) \otimes \mathbb{Q}$. The isotropy condition is the equation

$$
\hat{y} x=\hat{x} y .
$$

The existence of a splitting $X_{A} \rightarrow A$ in $\mathcal{A} \mathrm{b}_{\mathbb{Q}}$ is equivalent to the condition

$$
(\operatorname{End}(A) \otimes \mathbb{Q}) x+(\operatorname{Hom}(\hat{A}, A) \otimes \mathbb{Q}) y=\operatorname{End}(A) \otimes \mathbb{Q} .
$$

The pairs $\left(x_{1}, y_{1}\right)$ and $\left(x_{2}, y_{2}\right)$ define the same subvariety if and only if there exists an automorphism $\alpha$ of $A$ in $\mathcal{A} \mathrm{b}_{\mathbb{Q}}$ such that $x_{2}=x_{1} \alpha, y_{2}=y_{1} \alpha$. Thus, we obtain an identification of the set of Lagrangian subvarieties in $X_{A}$ with the set

$$
\mathbf{L G}(\mathbb{Q}):=\{(x, y) \mid \hat{y} x=\hat{x} y,(\star)\} /(x, y) \sim(x \alpha, y \alpha),
$$

where $x \in \operatorname{End}(A) \otimes \mathbb{Q}, y \in \operatorname{Hom}(A, \hat{A}) \otimes \mathbb{Q}$, and $\alpha \in(\operatorname{End}(A) \otimes \mathbb{Q})^{*}$. We denote by $(x: y) \in \mathbf{L G}(\mathbb{Q})$ the equivalence class of $(x, y) \in \operatorname{End}(A) \otimes \mathbb{Q} \oplus \operatorname{Hom}(A, \hat{A}) \otimes \mathbb{Q}$.

Fixing a polarization on $A$ we can identify $A$ with $\hat{A}$, so that the dualization gets replaced by the Rosati involution $x \mapsto x^{\prime}$ on $\mathcal{A}:=\operatorname{End}(A) \otimes \mathbb{Q}$. We claim that the set $\mathbf{L G}(\mathbb{Q})$ can be identified with the set of $\mathbb{Q}$-points of a certain homogeneous projective variety $\mathbf{L G}$ for the group $\mathbf{U}$ (a subvariety in the Grassmannian of right rank-1 $\mathcal{A}$-submodules in $\mathcal{A}^{2}$ ). Here the action of $\mathbf{U}$ on $\mathbf{L} \mathbf{G}$ is induced by the natural action of $\operatorname{End}\left(X_{A}\right)_{\mathbb{Q}}$ on pairs $(x, y)$ (viewed as column vectors). Consider the point $\left(0: \phi_{0}\right) \in \mathbf{L G}(\mathbb{Q})$, where $\phi_{0}: A \rightarrow \hat{A}$ is a polarization. (The corresponding Lagrangian is $0 \times \hat{A} \subset X_{A}$.) Note that the stabilizer subgroup of $\left(O: \phi_{0}\right)$ is the subgroup $\mathbf{P}^{-} \subset \mathbf{U}$ of lower-triangular matrices. Thus, we define

$$
\mathbf{L G}=\mathbf{L G}_{A}=\mathbf{U} / \mathbf{P}^{-} .
$$

The fact that the set (1.4.1) is indeed the set of $\mathbb{Q}$-points of $\mathbf{L G}$ follows from the transitivity of the action of $\mathbf{U}(\mathbb{Q})$ on the set of Lagrangian subvarieties that we will prove below (see Proposition 1.4.3).

We start with the following useful result.

\section{PROPOSITION 1.4.1}

For any collection of Lagrangian subvarieties $L_{1}, \ldots, L_{r} \subset X_{A}$ there exists an element $g \in \mathbf{U}(\mathbb{Z})$ such that all the Lagrangians $g L_{1}, \ldots, g L_{r}$ are transversal to $\{0\} \times \hat{A}$.

\section{Proof}

We use an argument similar to the first part of the proof of [31, Theorem 3.2.11]. Consider elements in $\mathbf{U}(\mathbb{Z})$ of the form $g_{n b}^{+}$for some polarization $b: \hat{A} \rightarrow A$, where $n \in \mathbb{Z}$. Then the condition that $g_{n b}^{+} L_{i}$ is transversal to $\{0\} \times \hat{A}$ is equivalent to $L_{i}$ being transversal to $g_{-n b}^{+}(\{0\} \times \hat{A})=\Gamma(-n b)$. By [31, Lemma 2.2.7(ii)], the latter transversality holds for all $n$ except for a finite number. 


\section{REMARK 1.4 .2}

The above proposition immediately implies that the subset $\mathbf{U}^{0} \cap \mathbf{U}(\mathbb{Z})$ of the group $\mathbf{U}(\mathbb{Z})$ is big (see Section 1.3). Indeed, for any given $g_{1}, \ldots, g_{r} \in \mathbf{U}(\mathbb{Z})$ consider the Lagrangian subvarieties $L_{i}=g_{i}(\{0\} \times \hat{A}) \subset X_{A}, i=1, \ldots, n$. Then we can find $g \in \mathbf{U}(\mathbb{Z})$ such that $g L_{i}=g g_{i}(\{0\} \times \hat{A})$ for $i=1, \ldots, n$ are transversal to $\{0\} \times \hat{A}$. Thus, we get $g g_{i} \in \mathbf{U}^{0}$ as required. The same proof works for any finite-index subgroup $\Gamma \subset \mathbf{U}(\mathbb{Z})$. The fact that $\mathbf{U}^{0} \cap \Gamma$ is a big subset of $\Gamma$ was stated in [26, Lemma 4.3]. However, the proof in [26, Lemma 4.3] was not correct: it relied on the absence of compact factors in $S \mathbf{U}(\mathbb{R})$, which is not always the case (see [12, Corollary 5.3.3]).

Lagrangian subvarieties in $X_{A}$, transversal to $0 \times \hat{A}$, are all graphs $\Gamma(f)$ of symmetric homomorphisms $f \in \operatorname{Hom}(A, \hat{A})^{+} \otimes \mathbb{Q}$ (see [31, Example 2.2.4]). This corresponds to points of $\mathbf{L G}(\mathbb{Q})$ of the form $(1: f)$, which are precisely $\mathbb{Q}$-points of the Zariski-open subset

$$
\mathrm{NS}(A)_{\mathbb{Q}} \simeq \mathbf{N}^{-} w_{\phi} \mathbf{P}^{-} / \mathbf{P}^{-} \subset \mathbf{L G},
$$

where $w_{\phi}$ is given by (1.3.1) and $\mathbf{N}^{-} \subset \mathbf{U}$ is the subgroup of strictly lowertriangular matrices. In other words, the subset (1.4.2) is just the $\mathbf{N}^{-}$-orbit of the point $(1: 0) \in \mathbf{L G}$.

\section{PROPOSITION 1.4.3}

(i) The action of $\mathbf{U}(\mathbb{Q})$ on the set of Lagrangian subvarieties in $X_{A}$ is transitive.

(ii) The action of $\mathbf{U}(\mathbb{R})$ on $\mathbf{L G}(\mathbb{R})$ is transitive.

Proof

(i) The subgroup $\mathbf{N}^{-}(\mathbb{Q}) \simeq \mathrm{NS}(A) \otimes \mathbb{Q}$ acts on the subset $\mathrm{NS}(A)_{\mathbb{Q}} \subset \mathbf{L G}$ by translations, so the corresponding action on the set of $\mathbb{Q}$-points is transitive. By Proposition 1.4.1, any point of $\mathbf{L G}(\mathbb{Q})$ is obtained from a $\mathbb{Q}$-point of this subset by an action of $\mathbf{U}(\mathbb{Z})$, so the required transitivity follows.

(ii) As is well known, it suffices to check the triviality of the kernel of the map of Galois cohomology $H^{1}\left(\mathbb{R}, \mathbf{P}^{-}\right) \rightarrow H^{1}(\mathbb{R}, \mathbf{U})$. Since $\mathbf{P}^{-}$is a semidirect product of $\prod_{i} \mathrm{GL}_{n_{i}}\left(D_{i}\right)$ (where the $D_{i}$ 's are skew fields) and of $\mathbb{G}_{a}^{n}$, in fact, the set $H^{1}\left(\mathbb{R}, \mathbf{P}^{-}\right)$is trivial.

The description (1.4.1) of $\mathbb{Q}$-points of $\mathbf{L G}$ can be extended to a similar description of $\mathbf{L G}(F)$, where $F=\mathbb{R}$ or $\mathbb{C}$, so we can still use homogeneous coordinates $(x: y)$, where $x \in \operatorname{End}(A) \otimes F$ and $y \in \operatorname{Hom}(A, \hat{A}) \otimes F$, to describe points of $\mathbf{L G}(F)$.

The complexified ample cone $D_{A} \subset \mathrm{NS}(A) \otimes \mathbb{C}$ is a Hermitian symmetric space (a tube domain) with the group of isometries $\mathbf{U}(\mathbb{R})$ (see [21, Section 5], [12, Section 8]). Namely, the group $\mathbf{U}(\mathbb{R})$ acts on $D_{A}$ by

$$
g(\omega)=(c+d \omega)(a+b \omega)^{-1} .
$$


This action is well defined $\operatorname{since} \operatorname{deg}(a+b \omega) \neq 0$ for $\omega \in D_{A}$ (see [12, Lemma A3]). Furthermore, it is transitive, and the stabilizer of a point $\omega \in D_{A}$ is a maximal compact subgroup of $\mathbf{U}(\mathbb{R})$ (see [12, Theorem A1]). Also, the natural embedding

$$
D_{A} \hookrightarrow \mathbf{L G}(\mathbb{C}): \omega \mapsto(1: \omega)
$$

is $\mathbf{U}(\mathbb{R})$-equivariant.

\section{LI functors and central extensions}

\subsection{Ll objects and functors}

Recall that every object $K \subset D^{b}(A \times A)$ gives rise to a functor of Fourier-Mukai type

$$
\Phi_{K}: D^{b}(A) \rightarrow D^{b}(A): F \mapsto R p_{2 *}\left(p_{1}^{*} F \otimes^{\mathbb{L}} K\right),
$$

where $p_{1}$ and $p_{2}$ are projections of $A \times A$ to its factors. (We refer to $K$ as the kernel of the functor $\Phi_{K}$.) The composition $\Phi_{K_{1}} \circ \Phi_{K_{2}}$ corresponds to the convolution of kernels $K_{2}{ }^{\circ} K_{1}$ (see [20]; our notation is as in [30]).

Recall that in [31] we have extended the relation between autoequivalences of $D^{b}(A)$ and the group $\mathbf{U}(\mathbb{Z})$ (see [23], [25]) to a construction of endofunctors of $D^{b}(A)$ (given by kernels on $A \times A$ ) associated with elements of $\mathbf{U}(\mathbb{Q})$ suitably enhanced. Namely, to every element $g \in \mathbf{U}(\mathbb{Q})$ we associate its graph $L(g) \subset$ $X_{A} \times X_{A}$, which we view as a Lagrangian subvariety in $X_{A} \times X_{A}$ with respect to the symplectic self-duality $\left(-\eta_{A}\right) \times \eta_{A}$ (see [31, Section 3.1]). The corresponding kernel on $A \times A$ is constructed as a generator of the subcategory of $L(g)$-invariants with respect to the action of $X_{A} \times X_{A}$ on $D^{b}(A \times A)$.

More precisely, every Lagrangian subvariety $L \subset X_{A}$ can be equipped with a line bundle $\alpha$ such that we have an isomorphism of line bundles on $L \times L$

$$
\alpha_{l_{1}+l_{2}} \otimes \alpha_{l_{1}}^{-1} \otimes \alpha_{l_{2}}^{-1} \simeq \mathcal{P}_{p_{A}\left(l_{1}\right), p_{\hat{A}}\left(l_{2}\right)},
$$

where $p_{A}: L \rightarrow A$ and $p_{\hat{A}}: L \rightarrow \hat{A}$ are the projections and $\mathcal{P}$ is the Poincaré bundle on $A \times \hat{A}$. We refer to $(L, \alpha)$ as Lagrangian pair. For every such pair $(L, \alpha)$ there exists a unique up to an isomorphism endosimple coherent sheaf $S_{L, \alpha}$ on $A$ together with an isomorphism

$$
\left(S_{L, \alpha}\right)_{x+p_{A}(l)} \otimes \mathcal{P}_{x, p_{\hat{A}}(l)} \otimes \alpha_{l} \simeq\left(S_{L, \alpha}\right)_{x}
$$

on $L \times A$ (where $l \in L, x \in A$ ), satisfying a certain natural compatibility condition. We view this condition as invariance with respect to the lifting of $L$ to the Heisenberg groupoid $\mathbf{H}=\mathbf{H}_{A}$, acting on $D^{b}(A)$ (and on $D^{b}(A \times S)$ for any scheme $S$ ). By definition, $\mathbf{H}$ is a Picard groupoid extension of $X_{A}$ by the stack of line bundles, so its objects over a scheme $S$ are pairs: a point $(x, \xi) \in X_{A}(S)$ and a line bundle $\mathcal{L}$ on $S$. The group operation is determined by

$$
\left(x_{1}, \xi_{1}\right) \cdot\left(x_{2}, \xi_{2}\right)=\mathcal{P}_{x_{1}, \xi_{2}} \cdot\left(x_{1}+x_{2}, \xi_{1}+\xi_{2}\right) .
$$

The action of $(x, \xi) \in X_{A}(S) \subset \mathbf{H}(S)$ on $D^{b}(A \times S)$ is given by the functors

$$
F \mapsto T_{(x, \xi)}(F)=\mathcal{P}_{\xi} \otimes t_{x}^{*} F,
$$


where $\mathcal{P}_{\xi}$ is the line bundle on $A \times S$ corresponding to $\xi \in \hat{A}(S)$. A choice of a line bundle $\alpha$ satisfying (2.1.1) gives a lifting of $L$ to a subgroup of $\mathbf{H}$, and the left-hand side of (2.1.2) is the result of the action of $l \in L$ on $S_{L, \alpha}$.

\section{DEFINITION 2.1.1}

$L I$ objects are cohomologically pure nonzero objects in $D^{b}(A)$ that can be equipped with $(L, \alpha)$-invariance isomorphism (2.1.2) for some $(L, \alpha)$ as above. In fact, they are all of the form $S_{L, \alpha}^{\oplus n}[m]$ for some $(L, \alpha), n \in \mathbb{N}$, and $m \in \mathbb{Z}$ (see [31, Theorem 2.4.5]). Let $\mathrm{SH}^{\mathrm{LI}}(A)$ denote the set of isomorphism classes of LI objects on $A$. In this paper we work mostly with the set $\overline{\mathrm{SH}}^{\mathrm{LI}}(A)$ of LI objects viewed up to the action of $\mathbf{H}(k)$, that is, up to translations and tensoring with line bundles in $\operatorname{Pic}^{0}(A)$. We will refer to this equivalence relation as $\mathbf{H}$-equivalence.

We will use the notation $N \cdot F:=F^{\oplus N}$ for an LI object $F$. This defines an action of the multiplicative monoid $\mathbb{N}^{*}$ on $\overline{\mathrm{SH}}^{\mathrm{LI}}(A)$.

PROPOSITION 2.1.2

There is a well-defined map

$$
\mathbf{L G}(\mathbb{Q}) \rightarrow \overline{\mathrm{SH}}^{\mathrm{LI}}(A): L \mapsto S(L)
$$

sending a Lagrangian subvariety $L \subset X_{A}$ to the class of the $L I$ sheaf $S_{L, \alpha}$, where $(L, \alpha)$ is a Lagrangian pair extending $L$. The map

$$
\mathbf{L G}(\mathbb{Q}) \times \mathbb{N}^{*} \times \mathbb{Z} \rightarrow \overline{\mathrm{SH}}^{\mathrm{LI}}(A):(L, N, n) \mapsto N \cdot S(L)[n]
$$

is a bijection of $\mathbb{N}^{*} \times \mathbb{Z}$ sets.

\section{Proof}

The fact that $S(L)$ depends only on $L$ follows from [31, Lemma 2.4.2]. The second statement follows from [31, Theorem 2.4.5] about the structure of the category of $(L, \alpha)$-invariants in $D^{b}(A)$ and [31, Corollary 2.4.11] stating that $L$ can be recovered from $S_{L, \alpha}$.

Recall that for an element $\phi \in \mathrm{NS}(A) \otimes \mathbb{Q} \simeq \operatorname{Hom}(A, \hat{A})^{+} \otimes \mathbb{Q}$ the graph $\Gamma(\phi)$ is a Lagrangian subvariety of $X_{A}$. Furthermore, these graphs are precisely all Lagrangians $L \subset X_{A}$ such that the projection $L \rightarrow A$ is an isogeny. The sheaf $S_{\Gamma(\phi), \alpha}$ associated with a Lagrangian pair $(\Gamma(\phi), \alpha)$ is a simple semihomogeneous vector bundle with $c_{1} / \mathrm{rk}=\phi$ (see [19]). For $\phi \in \mathrm{NS}(A) \otimes \mathbb{Q}$ we denote the $\mathbf{H}$ equivalence class of this bundle by

$$
V_{\phi}=S(\Gamma(\phi)) .
$$

The above construction of LI sheaves can be applied to Lagrangian subvarieties $L \subset X_{A} \times X_{B}$ for a pair of abelian varieties $A$ and $B$, where we use the symplectic self-duality $\left(-\eta_{A}\right) \times \eta_{B}$ of $X_{A} \times X_{B}$. We refer to the corresponding Lagrangian pairs $(L, \alpha)$ as Lagrangian correspondences from $X_{A}$ to $X_{B}$. The 
obtained LI sheaves $S_{L, \alpha}$ on $A \times B$ can be used as kernels of $L I$ functors

$$
\Phi_{L, \alpha}:=\Phi_{S_{L, \alpha}}: D^{b}(A) \rightarrow D^{b}(B) .
$$

The key property of these functors is that we have canonical isomorphisms

$$
\Phi_{L, \alpha} \circ T_{p_{1}(l)} \simeq \alpha_{l} \otimes T_{p_{2}(l)} \circ \Phi_{L, \alpha}
$$

for $l \in L$, where $p_{1}, p_{2}: L \rightarrow X_{A}$ are two projections. Note that every exact equivalence $D^{b}(A) \rightarrow D^{b}(B)$ is given by such an LI functor with $L$ being the graph of a symplectic isomorphism $X_{A} \simeq X_{B}$ (see [23]).

Let $p_{A B}: L \rightarrow A \times B, p_{A \hat{A}}: L \rightarrow A \times \hat{A}$, and $p_{B \hat{B}}: L \rightarrow B \times \hat{B}$ be the projections. The line bundle $\alpha$ can always be chosen in such a way that its restriction to the connected component of zero in $\operatorname{ker}\left(p_{A B}\right)$ is trivial. In this case $S_{L, \alpha}$ is a direct summand in (see [31, Lemma 3.2.5])

$$
p_{A B *}\left(\alpha^{-1} \otimes p_{A \hat{A}}^{*} \mathcal{P}^{-1} \otimes p_{B \hat{B}}^{*} \mathcal{P}\right) .
$$

In the case when $p_{A B}$ is an isogeny the finite group scheme $\operatorname{ker}\left(p_{A B}\right)$ has a canonical central extension $H_{L}$ by $\mathbb{G}_{m}$ with the underlying line bundle $\left.\alpha\right|_{\operatorname{ker}\left(p_{A B}\right)}$. Furthermore, $H_{L}$ is a Heisenberg group scheme, and (2.1.6) has a natural $H_{L^{-}}$ action, so that

$$
S_{L, \alpha}=p_{A B *}\left(\alpha^{-1} \otimes p_{A \hat{A}}^{*} \mathcal{P}^{-1} \otimes p_{B \hat{B}}^{*} \mathcal{P}\right)^{I}
$$

for a maximal isotropic subgroup $I \subset \operatorname{ker}\left(p_{A B}\right)$ lifted to $H_{L}$. It follows from the theory of weight-one representations of Heisenberg groups that taking $I$ invariants reduces the rank by the factor $\left|\operatorname{ker}\left(p_{A B}\right)\right|^{1 / 2}$, so we get

$$
\operatorname{rk} S_{L, \alpha}=\operatorname{deg}\left(p_{A B}: L \rightarrow A \times B\right)^{1 / 2} .
$$

In particular, for $B=0$ we get

$$
\operatorname{rk} V_{\phi}=\operatorname{deg}\left(p_{A}: \Gamma(\phi) \rightarrow A\right)^{1 / 2} .
$$

\section{EXAMPLE 2.1.3}

The functor of tensoring with a line bundle $L$ on $D^{b}(A)$ commutes with the action of $\hat{A}$ and satisfies

$$
L \otimes\left(t_{x}^{*} F\right) \simeq \mathcal{P}_{-\phi_{L}(x)} \otimes t_{x}^{*}(L \otimes F) .
$$

In fact, it is the LI functor corresponding to $g_{-\phi_{L}}=\left(\begin{array}{cc}\text { id } & 0 \\ -\phi_{L} & \text { id }\end{array}\right)$. More generally, for $\phi \in \mathrm{NS}(A) \otimes \mathbb{Q}$ the LI functor corresponding to the element $g_{-\phi} \in \mathbf{N}^{-}(\mathbb{Q})$ is the functor of tensoring with the semihomogeneous vector bundle $V_{\phi}$ (up to H-equivalence).

The above construction gives a map

$$
\mathbf{U}(\mathbb{Q}) \rightarrow \overline{\mathrm{SH}}^{\mathrm{LI}}(A \times A): g \rightarrow S(g)=S(L(g)) .
$$

We denote by $\Phi_{g} \in \operatorname{Fun}\left(D^{b}(A), D^{b}(A)\right) / \mathbf{H}$ the functor associated with the kernel $S(g)$, defined up to composing with a functor of the form $T_{(x, \xi)},(x, \xi) \in X_{A}$ (on 
either side). For each $(x, \xi) \in X_{A}$ we have (noncanonical) isomorphisms

$$
\Phi_{g} \circ T_{N(x, \xi)} \simeq T_{N g(x, \xi)} \circ \Phi_{g}
$$

where $N$ is such that $N g \in \operatorname{End}\left(X_{A}\right)$.

Note that we have a well-defined homomorphism induced by $\Phi_{g}$

$$
\rho(g): \mathcal{N}(A) \rightarrow \mathcal{N}(A) .
$$

\section{DEFINITION 2.1.4}

Let $F$ be a cohomologically pure object of $D^{b}(A)$, and let $G$ be an endosimple LI object. We write

$$
F \equiv N \cdot G
$$

if there exists $n \in \mathbb{Z}$ such that $F[n]$ and $G[n]$ are sheaves and $F[n]$ has a filtration of length $N$ such that each consecutive quotient is $\mathbf{H}$-equivalent to $G[n]$. In the case of sheaves on $A \times A$ we will use the same notation for the relation between the corresponding endofunctors of $D^{b}(A)$.

One of the main results of [31] is the following calculation of the convolution of kernels (see [31, Theorem 3.3.4]):

$$
S\left(g_{2}\right) \circ_{A} S\left(g_{1}\right) \equiv N\left(g_{1}, g_{2}\right) \cdot S\left(g_{1} g_{2}\right)\left[\lambda\left(g_{1}, g_{2}\right)\right],
$$

for some 2-cocycles $N\left(g_{1}, g_{2}\right)$ and $\lambda\left(g_{1}, g_{2}\right)$ of $\mathbf{U}(\mathbb{Q})$ with values in $\mathbb{N}^{*}$ and $\mathbb{Z}$, respectively. ${ }^{\dagger}$ Furthermore, we have

$$
N\left(g_{1}, g_{2}\right)=\frac{q\left(L\left(g_{1}\right)\right)^{1 / 2} q\left(L\left(g_{2}\right)\right)^{1 / 2}}{q\left(L\left(g_{1} g_{2}\right)\right)^{1 / 2}}
$$

where

$$
q(g)=q(L(g))=\operatorname{deg}\left(p_{1}: L(g) \rightarrow X_{A}\right) .
$$

Also, for $g_{1}, g_{2} \in \mathbf{U}^{0}(\mathbb{Q})$ such that $g_{1} g_{2} \in \mathbf{U}^{0}(\mathbb{Q})$ one has

$$
\lambda\left(g_{1}, g_{2}\right)=-i\left(b\left(g_{1}\right)^{-1} b\left(g_{1} g_{2}\right) b\left(g_{2}\right)^{-1}\right) .
$$

Note that in order for the right-hand side to be well defined the argument of $i(\cdot)$ should be symmetric. This indeed follows from the equality

$$
b_{1}^{-1}\left(a_{1} b_{2}+b_{1} d_{2}\right) b_{2}^{-1}=b_{1}^{-1} a_{1}+d_{2} b_{2}^{-1},
$$

where we use the usual notation for the entries of $g_{1}$ and $g_{2}$.

\section{DEFINITION 2.1.5}

We denote by $\widetilde{\mathbf{U}(\mathbb{Q})}$ the central extension of $\mathbf{U}(\mathbb{Q})$ by $\mathbb{Z}$ associated with the

${ }^{\dagger}$ In [31, Theorem 3.3.4] we made the assumption that $\operatorname{char}(k)=0$, which implies a stronger statement: the left-hand side of (2.1.11) is a direct sum of objects $\mathbf{H}$-equivalent to the righthand side. It is easy to see that the same argument in the positive characteristic case gives a filtration instead of a direct sum. 
2-cocycle $\lambda(\cdot, \cdot)$. Explicitly $\widetilde{\mathbf{U}(\mathbb{Q})}=\mathbf{U}(\mathbb{Q}) \times \mathbb{Z}$ with the product

$$
\left(g_{1}, n_{1}\right) \cdot\left(g_{2}, n_{2}\right)=\left(g_{1} g_{2}, n_{1}+n_{2}+\lambda\left(g_{1}, g_{2}\right)\right) .
$$

Note that, since the subset $\mathbf{U}^{0}(\mathbb{Q}) \subset \mathbf{U}(\mathbb{Q})$ is big (see Lemma 1.3.4), by Lemma 1.3.3(ii), the formula (2.1.14) determines the extension $\widetilde{\mathbf{U}(\mathbb{Q})}$ uniquely up to a unique isomorphism.

Let us denote by $\overline{\mathrm{SH}}^{\mathrm{LI}}(A) / \mathbb{N}^{*}$ the set of equivalence classes with respect to the equivalence relation generated by $F \sim N \cdot F$ for some $N \in \mathbb{N}^{*}$. By (2.1.11), the map $g \mapsto S(g) \bmod \mathbb{N}^{*}$ defines a homomorphism of monoids

$$
\widetilde{\mathrm{U}(\mathbb{Q})} \rightarrow \overline{\mathrm{SH}}^{\mathrm{LI}}(A \times A)^{o p} / \mathbb{N}^{*},
$$

and hence a homomorphism of monoids

$$
\widetilde{\mathbf{U}(\mathbb{Q})} \rightarrow \operatorname{Fun}\left(D^{b}(A), D^{b}(A)\right) /\left(\mathbf{H} \times \mathbb{N}^{*}\right): g \mapsto \Phi_{g},
$$

where on the right we consider functors up to $\mathbf{H}$-equivalence and up to replacing $\Phi$ with $N \cdot \Phi=\Phi^{\oplus N}$.

On the level of numerical Grothendieck groups we can eliminate taking quotients by $\mathbb{N}^{*}$. Namely, let us set for $g \in \mathbf{U}(\mathbb{Q})$

$$
\hat{\rho}(g)=\frac{\rho(g)}{q(g)^{1 / 2}}: \mathcal{N}(A) \otimes \mathbb{R} \rightarrow \mathcal{N}(A) \otimes \mathbb{R} .
$$

Then from (2.1.11) and (2.1.12) we derive that

$$
\hat{\rho}\left(g_{1}\right) \hat{\rho}\left(g_{2}\right)=(-1)^{\lambda\left(g_{1}, g_{2}\right)} \hat{\rho}\left(g_{1} g_{2}\right),
$$

where $g_{1}, g_{2} \in \mathbf{U}(\mathbb{Q})$. Thus, $\hat{\rho}$ defines a homomorphism from $\widetilde{\mathbf{U}(\mathbb{Q})}$ to $\operatorname{GL}(\mathcal{N}(A) \otimes$ $\mathbb{R})$, trivial on the central subgroup $2 \mathbb{Z} \subset \mathbb{Z} \subset \widehat{\mathbf{U}(\mathbb{Q})}$. Note that the quotient $\widehat{\mathbf{U}(\mathbb{Q})} / 2 \mathbb{Z}$ is a double cover of $\mathbf{U}(\mathbb{Q})$. Below we introduce an algebraic structure on this double cover and show that $\hat{\rho}$ is induced by an algebraic homomorphism defined over $\mathbb{R}$ (see Sections 2.3 and 2.5).

\subsection{Splittings over subgroups}

We are going to define a splitting of the central extension $\widetilde{\mathbf{U}(\mathbb{Q})} \rightarrow \mathbf{U}(\mathbb{Q})$ over the parabolic subgroup $\mathbf{P}^{+}(\mathbb{Q}) \subset \mathbf{U}(\mathbb{Q})$ of lower-triangular matrices (resp., over the subgroup $\mathbf{P}^{-}(\mathbb{Q})$ of upper-triangular matrices). Note that $\mathbf{P}^{+}(\mathbb{Q})$ is a semidirect product of the subgroups of strictly upper-triangular matrices $\mathbf{N}^{+}(\mathbb{Q}) \simeq \mathrm{NS}(A) \otimes$ $\mathbb{Q}$ and of diagonal matrices $\mathbf{T}(\mathbb{Q}) \simeq(\operatorname{End}(A) \otimes \mathbb{Q})^{*}$.

\section{PROPOSITION 2.2.1}

(i) There exist unique liftings of the subgroups $\mathbf{N}^{+}(\mathbb{Q})$ and $\mathbf{N}^{-}(\mathbb{Q})$ to $\widetilde{\mathbf{U}(\mathbb{Q})}$. The lifting of the element $g_{\phi}^{+}=\left(\begin{array}{ll}1 & \phi \\ 0 & 1\end{array}\right)$, where $\phi \in \operatorname{NS}^{0}(A, \mathbb{Q})$, is given by $\left(g_{\phi}^{+}, i(\phi)\right) \in \widehat{\mathbf{U}(\mathbb{Q})}$. The lifting of the element $g_{\phi}^{-}=\left(\begin{array}{ll}1 & 0 \\ \phi & 1\end{array}\right)$, where $\phi \in \operatorname{NS}^{0}(A, \mathbb{Q})$, is given by $\left(g_{\phi}^{-}, 0\right) \in \widetilde{\mathbf{U}(\mathbb{Q})}$. The corresponding functor $\Phi_{g_{\phi}^{-}}$(defined up to 
$\mathbf{H}$-equivalence) is given by tensoring with the semihomogeneous bundle $V_{-\phi}$ (see (2.1.4)).

(ii) For $t=t_{a}=\left(\begin{array}{cc}a^{-1} & 0 \\ 0 & \hat{a}\end{array}\right) \in \mathbf{T}(\mathbb{Q})$ we have (up to $\mathbf{H}$-equivalence)

$$
S(t)=\mathcal{O}_{B}
$$

for some abelian subvariety $B \subset A \times A$ such that the two projections $p, q: B \rightarrow A$ are isogenies. Hence, the functor $\Phi_{t}$ is of the form $q_{*} p^{*}$ (up to $\mathbf{H}$-equivalence).

(iii) For any $t \in \mathbf{T}(\mathbb{Q})$ and $g \in \mathbf{U}(\mathbb{Q})$ one has $\lambda(t, g)=0$.

\section{Proof}

(i) The uniqueness of the liftings follows from the fact that there are no nontrivial homomorphisms from a $\mathbb{Q}$-vector space to $\mathbb{Z}$. Thus, to check the formula for the lifting of $g_{\phi}^{+}$we have to check that

$$
S\left(g_{\phi_{2}}^{+}\right) \circ S\left(g_{\phi_{1}}^{+}\right)=S\left(g_{\phi_{1}+\phi_{2}}^{+}\right)\left[i\left(\phi_{1}+\phi_{2}\right)-i\left(\phi_{1}\right)-i\left(\phi_{2}\right)\right],
$$

for $\phi_{1}, \phi_{2} \in \mathrm{NS}^{0}(A, \mathbb{Q})$ such that $\phi_{1}+\phi_{2} \in \mathrm{NS}^{0}(A, \mathbb{Q})$. But

$$
\lambda\left(g_{\phi_{1}}^{+}, g_{\phi_{2}}^{+}\right)=-i\left(\phi_{1}^{-1}\left(\phi_{1}+\phi_{2}\right) \phi_{2}^{-1}\right)=-i\left(\phi_{1}^{-1}+\phi_{2}^{-1}\right),
$$

so we are reduced to showing that

$$
i\left(\phi_{1}^{-1}+\phi_{2}^{-1}\right)=i\left(\phi_{1}\right)+i\left(\phi_{2}\right)-i\left(\phi_{1}+\phi_{2}\right) .
$$

Since

$$
i\left(\phi_{1}^{-1}+\phi_{2}^{-1}\right)=i\left(\phi_{1}\left(\phi_{1}^{-1}+\phi_{2}^{-1}\right) \phi_{1}\right)=i\left(\phi_{1}+\phi_{1} \phi_{2}^{-1} \phi_{1}\right),
$$

this follows from [27, Proposition 15.8] (taking into account that $i(-x)=g-$ $i(x))$.

Since the composition of functors $\otimes V_{\phi_{1}}$ and $\otimes V_{\phi_{2}}$ is again tensoring with a bundle that has a filtration with consecutive quotients $\mathbf{H}$-equivalent to $V_{\phi_{1}+\phi_{2}}$, the assertion about the lifting of $g_{\phi}^{-}$follows (cf. Example 2.1.3).

(ii) Assume first that $a \in \operatorname{End}(A)$. Then $L\left(t_{a}\right) \simeq A \times \hat{A}$, and its embedding into $X_{A} \times X_{A}$ is given by

$$
(x, \xi) \mapsto(a x, \xi, x, \hat{a} \xi) .
$$

This implies that $\left(L\left(t_{a}\right), \mathcal{O}\right)$ is a Lagrangian correspondence from $X_{A}$ to itself, so (2.1.6) in this case gives that

$$
S_{L\left(t_{a}\right), \mathcal{O}} \simeq\left(a, \mathrm{id}_{A}\right)_{*} \mathcal{O}_{A},
$$

and the corresponding functor $\Phi_{t_{a}}$ is the pullback functor $a^{*}$. Similarly, if $a^{-1} \in$ $\operatorname{End}(A)$, then

$$
S_{L\left(t_{a}\right), \mathcal{O}} \simeq\left(\operatorname{id}_{A}, a^{-1}\right)_{*} \mathcal{O}_{A},
$$

and the corresponding functor $\Phi_{t_{a}}$ is the pushforward functor $\left(a^{-1}\right)_{*}$. The general case is obtained by combining these two. 
(iii) We have to check that the convolution $S(g) \circ_{A} S(t)$ is a sheaf. Indeed, using the form of $S(t)$ from (ii) we obtain that

$$
S(g) \circ_{A} S(t) \simeq\left(\operatorname{id}_{A} \times q\right)_{*}\left(\operatorname{id}_{A} \times p\right)^{*} S(g),
$$

where $p, q: B \rightarrow A$ are isogenies.

\section{COROLLARY 2.2.2}

There is a unique splitting of the central extension $\widehat{\mathbf{U}(\mathbb{Q})} \rightarrow \mathbf{U}(\mathbb{Q})$ over $\mathbf{P}^{+}(\mathbb{Q}) \subset$ $\mathbf{U}(\mathbb{Q})$ (resp., over $\mathbf{P}^{-}(\mathbb{Q})$ ), which maps $t \in \mathbf{T}(\mathbb{Q})$ to $(t, 0) \in \widehat{\mathbf{U}(\mathbb{Q}) \text {. }}$

\subsection{Identifying central extensions}

Recall that $D_{A} \subset \mathrm{NS}(A) \otimes \mathbb{C} \simeq \operatorname{Hom}(A, \hat{A})^{+} \otimes \mathbb{C}$ denotes the complexified ample cone of $A$.

Consider the function $\Delta: \mathbf{U}(\mathbb{R}) \rightarrow \mathcal{O}^{*}\left(D_{A}\right)$ given by

$$
g=\left(\begin{array}{ll}
a & b \\
c & d
\end{array}\right) \mapsto \Delta(g)(\omega)=\operatorname{deg}(a+b \omega),
$$

where $\omega \in D_{A}$.

\section{LEMMA 2.3.1}

For $g_{1}, g_{2} \in \mathbf{U}(\mathbb{R})$ one has

$$
\Delta\left(g_{1} g_{2}\right)(\omega)=\Delta\left(g_{1}\right)\left(g_{2}(\omega)\right) \cdot \Delta\left(g_{2}\right)(\omega) ;
$$

that is, $\Delta$ is a 1-cocycle.

Proof

This follows from the identity

$$
a+b \omega=\left(a_{1}+b_{1} g_{2}(\omega)\right)\left(a_{2}+b_{2} \omega\right),
$$

where $g_{i}=\left(\begin{array}{ll}a_{i} & b_{i} \\ c_{i} & d_{i}\end{array}\right)$ for $i=1,2$ and $g_{1} g_{2}=\left(\begin{array}{ll}a & b \\ c & d\end{array}\right)$.

Since $D_{A}$ is contractible, we have an exact sequence of $\mathbf{U}(\mathbb{R})$-modules

$$
0 \rightarrow \mathbb{Z} \rightarrow \mathcal{O}\left(D_{A}\right) \stackrel{\exp (2 \pi i \cdot ?)}{\longrightarrow} \mathcal{O}^{*}\left(D_{A}\right) \rightarrow 0
$$

Applying the boundary homomorphism $H^{1}(\mathbf{U}(\mathbb{R})) \rightarrow H^{2}(\mathbf{U}(\mathbb{Z}))$ to the 1-cocycle $\Delta(g)^{-1}$ we obtain a central extension $U^{\Delta}$ of $\mathbf{U}(\mathbb{R})$ by $\mathbb{Z}$. Explicitly,

$$
U^{\Delta}=\left\{(g, f) \in \mathbf{U}(\mathbb{R}) \times \mathcal{O}\left(D_{A}\right) \mid \Delta(g)=\exp (-2 \pi i f)\right\} .
$$

The multiplication rule on $U^{\Delta}$ uses the cocycle condition on $\Delta$ : we set

$$
\left(g_{1}, f_{1}\right) \cdot\left(g_{2}, f_{2}\right)=\left(g_{1} g_{2}, f_{1}\left(g_{2}(\cdot)\right)+f_{2}\right) .
$$

\section{THEOREM 2.3.2}

There is a homomorphism $\iota: \widetilde{\mathbf{U}(\mathbb{Q})} \rightarrow U^{\Delta}$, lifting the natural embedding $\mathbf{U}(\mathbb{Q}) \rightarrow$ $\mathbf{U}(\mathbb{R})$ and sending $n \in \mathbb{Z} \subset \widehat{\mathbf{U}(\mathbb{Q})}$ to $(1, n) \in U^{\Delta}$. This homomorphism is uniquely 
characterized by the condition that for $g \in \mathbf{U}^{0}(\mathbb{Q})$ one has

$$
\iota(g, 0)=(g, f)
$$

where

$$
\lim _{n \rightarrow \infty} \operatorname{Re} f(i n H)=-\frac{g}{2}
$$

for any ample class $H$.

\section{Proof}

First, we are going to define a section $\sigma: \mathbf{U}^{0}(\mathbb{R}) \rightarrow U^{\Delta}$ of the projection $U^{\Delta} \rightarrow$ $\mathbf{U}(\mathbb{R})$ over the open subset $\mathbf{U}^{0}(\mathbb{R}) \subset \mathbf{U}(\mathbb{R})$ consisting of $g$ with $\operatorname{deg}(b(g)) \neq 0$. Note that for $g=\left(\begin{array}{ll}a & b \\ c & d\end{array}\right) \in \mathbf{U}^{0}(\mathbb{R})$ one has

$$
\Delta(g)(\omega)=\operatorname{deg}(a+b \omega)=\operatorname{deg}(b) \cdot \operatorname{deg}\left(b^{-1} a+\omega\right) .
$$

Since $\operatorname{deg}(b)>0$, to define $\sigma(g)=\left(g, f_{g}^{\sigma}\right)$ amounts to choosing a branch of the argument for $\operatorname{deg}\left(b^{-1} a+\omega\right)^{-1}$. Let us choose the branch of the argument of $\operatorname{deg}\left(b^{-1} a+\omega\right)$ in such a way that

$$
\lim _{n \rightarrow+\infty} \operatorname{Arg}\left(\operatorname{deg}\left(b^{-1} a+i n H\right)\right)=\pi \cdot g,
$$

where $H$ is an ample class, and set $\operatorname{Arg}\left(\Delta(g)(\omega)^{-1}\right)=-\operatorname{Arg}\left(\operatorname{deg}\left(b^{-1} a+\omega\right)\right)$. Then we set $\iota(g, 0)=\sigma(g)$ for $g \in \mathbf{U}^{0}(\mathbb{Q})$. Since $\mathbf{U}^{0}(\mathbb{Q})$ is big in $\mathbf{U}(\mathbb{Q})$, by Lemma 1.3.3, it remains to show that for $g_{1}, g_{2} \in \mathbf{U}^{0}(\mathbb{Q})$ such that $g_{1} g_{2} \in \mathbf{U}^{0}(\mathbb{Q})$ one has

$$
\sigma\left(g_{1}\right) \sigma\left(g_{2}\right)=\sigma\left(g_{1} g_{2}\right) \cdot\left(1, \lambda\left(g_{1}, g_{2}\right)\right)
$$

In other words, we have to check that

$$
f_{g_{1}}^{\sigma}\left(g_{2}(\omega)\right)+f_{g_{2}}^{\sigma}(\omega)=f_{g_{1} g_{2}}^{\sigma}(\omega)+\lambda\left(g_{1}, g_{2}\right)
$$

or, equivalently, that with the above choice of $\operatorname{Arg}(\Delta(g))$ one has

$$
\begin{array}{r}
\operatorname{Arg}\left(\Delta\left(g_{1}\right)\left(g_{2}(\omega)\right)\right)+\operatorname{Arg}\left(\Delta\left(g_{2}\right)(\omega)\right) \\
\quad=\operatorname{Arg}\left(\Delta\left(g_{1} g_{2}\right)(\omega)\right)-2 \pi \cdot \lambda\left(g_{1}, g_{2}\right) .
\end{array}
$$

It is enough to check the equality of the limits of both sides for $\omega=i n H$ as $n$ goes to infinity (where $H$ is an ample class). Let $g_{i}=\left(\begin{array}{cc}a_{i} & b_{i} \\ c_{i} & d_{i}\end{array}\right)$ for $i=1,2$. Note that

$$
\lim _{n \rightarrow \infty} g_{2}(i n H)=d_{2} b_{2}^{-1}
$$

Thus, (2.3.2) reduces to the equality

$$
\operatorname{Arg}\left(\Delta\left(g_{1}\right)\left(d_{2} b_{2}^{-1}\right)\right)=-2 \pi \lambda\left(g_{1}, g_{2}\right)=i\left(b_{1}^{-1} b\left(g_{1} g_{2}\right) b_{2}^{-1}\right) .
$$

But

$$
\begin{aligned}
\operatorname{Arg}\left(\Delta\left(g_{1}\right)\left(d_{2} b_{2}^{-1}\right)\right) & =\operatorname{Arg}\left(\operatorname{deg}\left(b_{1}^{-1} a_{1}+d_{2} b_{2}^{-1}\right)\right)=\operatorname{Arg}\left(\operatorname{deg}\left(b_{1}^{-1} b\left(g_{1} g_{2}\right) b_{2}^{-1}\right)\right) \\
& =2 \pi \cdot i\left(b_{1}^{-1} b\left(g_{1} g_{2}\right) b_{2}^{-1}\right)
\end{aligned}
$$

by Corollary 1.2.2. 
The central extension $U^{\Delta} \rightarrow \mathbf{U}(\mathbb{R})$ has a natural continuous splitting over the subgroup $\mathbf{P}^{-}(\mathbb{R}) \subset \mathbf{U}(\mathbb{R})$. Indeed, for $g \in \mathbf{P}^{-}(\mathbb{R})$ we have $\Delta(g)=\operatorname{deg}(a)>0$, so we can lift $g$ to

$$
\sigma_{\mathbf{P}^{-}}(g)=\left(g,-\frac{1}{2 \pi i} \log (\operatorname{deg}(a))\right)
$$

where we choose $\log (\operatorname{deg}(a))$ to be in $\mathbb{R}$. The following result will be useful for us later.

\section{LEMMA 2.3.3}

The restriction of the above lifting homomorphism $\mathbf{P}^{-}(\mathbb{R}) \rightarrow U^{\Delta}$ to $\mathbf{P}^{-}(\mathbb{Q})$ corresponds via $\iota$ to the lifting homomorphism $\mathbf{P}^{-}(\mathbb{Q}) \rightarrow \widehat{\mathbf{U}(\mathbb{Q})}$ considered in Corollary 2.2.2.

Proof

By Proposition 2.2.1(i), it is enough to check the compatibility of liftings on $\mathbf{T}(\mathbb{Q})$. In view of Proposition 2.2.1(iii) this follows from the equality

$$
\sigma_{\mathbf{P}^{-}}(t) \sigma(g)=\sigma(t g)
$$

for any $g \in \mathbf{U}^{0}(\mathbb{Q})$, where $\sigma: \mathbf{U}^{0}(\mathbb{R}) \rightarrow U^{\Delta}$ is the section used in the proof of Theorem 2.3.2.

Similarly, the extension $U^{\Delta} \rightarrow \mathbf{U}(\mathbb{R})$ has a natural continuous splitting over $\mathbf{P}^{+}(\mathbb{R})$, which is the same as before over $\mathbf{T}(\mathbb{R})$, and over $\mathbf{N}^{+}(\mathbb{R})$ is described as follows.

\section{LEMMA 2.3.4}

There is a unique splitting of $U^{\Delta} \rightarrow \mathbf{U}(\mathbb{R})$ over $\mathbf{N}^{+}(\mathbb{R}) \simeq \mathrm{NS}(\hat{A}, \mathbb{R})$ which is given by the branch of

$$
\left.\operatorname{Arg} \Delta^{-1}\right|_{\mathbf{N}^{+}(\mathbb{R})}=\operatorname{Arg} \operatorname{deg}(1+\psi \omega)
$$

that tends to 0 as $\omega \rightarrow 0$, where $\psi \in \mathrm{NS}(\hat{A}, \mathbb{R}) \simeq \operatorname{Hom}(\hat{A}, A)_{\mathbb{R}}^{+}$.

Proof

It is straightforward to check that this choice of argument gives a lifting. The uniqueness follows from the fact that there are no nontrivial homomorphisms from a real vector space to $\mathbb{Z}$.

Let us consider the induced double cover $U^{\Delta} / 2 \mathbb{Z} \rightarrow \mathbf{U}(\mathbb{R})$. We are going to introduce an algebraic structure on this group.

LEMMA 2.3.5

Consider a field extension $\mathbb{Q} \subset F$, where either $F=\mathbb{R}$ or $F$ is algebraically closed. Then for every $g=\left(\begin{array}{ll}a & b \\ c & d\end{array}\right) \in \mathbf{U}(F)$, the polynomial $\Delta(g)(\phi)=\operatorname{deg}(a+b \phi)$ on $\mathrm{NS}(A)(F)$ is a complete square (and is nonzero). 
Proof

For $g \in \mathbf{U}^{0}$ this follows from the equality

$$
\operatorname{deg}(a+b \phi)=\operatorname{deg}(b) \operatorname{deg}\left(b^{-1} a+\phi\right)=\operatorname{deg}(b) \chi\left(b^{-1} a+\phi\right)^{2}
$$

and the fact that $\operatorname{deg}(b) \geq 0$ in the case $F=\mathbb{R}$. Viewing (2.3.1) as an identity of rational functions on $\mathrm{NS}(A)$, we see that if $\Delta\left(g_{1}\right)$ and $\Delta\left(g_{2}\right)$ are complete squares, then $\Delta\left(g_{1} g_{2}\right)$ is a complete square as a rational function on $\mathrm{NS}(A)$ and, hence, as a polynomial.

\section{DEFINITION 2.3.6}

Let $\mathrm{Pol}_{\leq g}(\mathrm{NS}(A))$ denote the space of polynomials of degree at most $g$ on $\mathrm{NS}(A)$. We define a double covering Spin $=\operatorname{Spin}_{X_{A}} \rightarrow \mathbf{U}$ of algebraic groups over $\mathbb{Q}$ by setting

$$
\operatorname{Spin}=\left\{(g, f) \in \mathbf{U} \times \operatorname{Pol}_{\leq g}(\mathrm{NS}(A)) \mid \Delta(g)=f^{2}\right\}
$$

with the group law

$$
\left(g_{1}, f_{1}\right) \cdot\left(g_{2}, f_{2}\right)=\left(g_{1} g_{2}, f_{1}\left(g_{2}(\cdot)\right) \cdot f_{2}\right) .
$$

Here the rational function $f_{1}\left(g_{2}(\cdot)\right) \cdot f_{2}$ is actually a polynomial since its square is $\Delta\left(g_{1} g_{2}\right)$.

Note that by Lemma 2.3.5, the map $\pi: \operatorname{Spin}(\mathbb{R}) \rightarrow \mathbf{U}(\mathbb{R})$ is a double covering. We have a natural isomorphism of groups

$$
U^{\Delta} / 2 \mathbb{Z} \rightarrow \operatorname{Spin}(\mathbb{R}):(g, f) \mapsto(g, \exp (-\pi i f)) .
$$

We have two natural subgroups in $\operatorname{Spin}(\mathbb{R})$ :

$$
\mathbf{U}(\mathbb{Q})^{\text {spin }}=\pi^{-1}(\mathbf{U}(\mathbb{Q})), \quad \mathbf{U}(\mathbb{Z})^{\text {spin }}=\pi^{-1}(\mathbf{U}(\mathbb{Z})) .
$$

\section{LEMMA 2.3.7}

Consider the homomorphism

$$
\bar{\iota}: \widetilde{\mathbf{U}(\mathbb{Q})} / 2 \mathbb{Z} \stackrel{\sim}{\longrightarrow} \mathbf{U}(\mathbb{Q})^{\text {spin }} \subset \operatorname{Spin}(\mathbb{R})
$$

induced by $\iota: \widetilde{\mathbf{U}(\mathbb{Q})} \rightarrow U^{\Delta}$ (see Theorem 2.3.2) and the isomorphism (2.3.3). Then for $g=\left(\begin{array}{ll}a & b \\ c & d\end{array}\right) \in \mathbf{U}^{0}(\mathbb{Q})$ we have

$$
\bar{\iota}(g, 0)=\left(g, \sqrt{\operatorname{deg}(b)} \cdot \chi\left(b^{-1} a+\phi\right)\right)
$$

with $\sqrt{\operatorname{deg}(b)}>0$.

Proof

By Theorem 2.3.2, $\bar{\iota}(g, 0)=(g, f)$, where $f(\phi)$ is the square root of $\Delta(g)(\phi)=$ $\operatorname{deg}(b) \cdot \operatorname{deg}\left(b^{-1} a+\phi\right)$ with the property

$$
\lim _{n \rightarrow+\infty} \operatorname{Arg} f(i n H)=\frac{\pi \cdot g}{2} \bmod 2 \pi \mathbb{Z} .
$$

Since $\operatorname{Arg} \chi\left(b^{-1} a+i n H\right)$ has the same limit as $n \rightarrow+\infty$, the assertion follows. 


\section{REMARKS 2.3.8}

(1) If for a field extension $\mathbb{Q} \subset F$ there is a multiplicative norm $\mathrm{Nm}$ on $\operatorname{End}(A) \otimes F$ such that $\mathrm{Nm}^{2}=\operatorname{deg}$, then the map $g \mapsto(g, \mathrm{Nm}(a+b \omega))$ defines a splitting of the extension Spin $\rightarrow \mathbf{U}$ over $F$. For example, if $A=E^{n}$, where $E$ is an elliptic curve without complex multiplication, then $\operatorname{End}(A)=\operatorname{Mat}_{n}(\mathbb{Z})$ and $\operatorname{deg}\left([M]_{A}\right)=\operatorname{det}(M)^{2}$ for a matrix $M \in \operatorname{Mat}_{n}(\mathbb{Z})$. Hence, in this case the norm $\operatorname{det}(\cdot)$ gives a splitting of the spin covering.

(2) The group $\mathbf{U}(\mathbb{Z})^{\text {spin }}$ is exactly the group $U \operatorname{Spin}(A \times \hat{A})$ defined by Mukai in [21]. (The same group is denoted by $\operatorname{Spin}(A)$ in [12].)

Using the isomorphism $\widetilde{\mathbf{U}(\mathbb{Q})} / 2 \mathbb{Z} \simeq \mathbf{U}(\mathbb{Q})^{\text {spin }}$ we can define a homomorphism

$$
\hat{\rho}: \mathbf{U}(\mathbb{Q})^{\text {spin }} \rightarrow \mathrm{GL}(\mathcal{N}(A) \otimes \mathbb{R})
$$

such that $\hat{\rho}(\bar{l}(g, 0))$ is the operator $\hat{\rho}(g)$ (see $(2.1 .17))$.

\subsection{The action on LI objects}

Recall that with a Lagrangian correspondence from $X_{A}$ to itself extending a symplectic isomorphism $g: X_{A} \rightarrow X_{A}$ in $\mathcal{A} \mathrm{b}_{\mathbb{Q}}$ we associate an endofunctor $\Phi_{g}$ of $D^{b}(A)$, defined up to $\mathbf{H}$-equivalence (see Section 2.1). We are going to use these endofunctors to define an action of $\widetilde{\mathbf{U}(\mathbb{Q})}$ on some extension of $\overline{\mathrm{SH}}^{\mathrm{LI}}(A)$ (see Corollary 2.4.2).

\section{THEOREM 2.4.1}

(i) For an element $g \in \mathbf{U}(\mathbb{Q})$ and a Lagrangian subvariety $L \subset X_{A}$ we have

$$
\Phi_{g}(S(L)) \equiv N(g, L) \cdot S(g L)[\lambda(g, L)]
$$

for some $\lambda(g, L) \in \mathbb{Z}$ and $N(g, L) \in \mathbb{N}^{*}$, where we use Definition 2.1.4.

(ii) If $L=\Gamma(\phi)$ for an isogeny $\phi \in \operatorname{Hom}(A, \hat{A})^{+} \otimes \mathbb{Q}$ and if $g=\left(\begin{array}{ll}a & b \\ c & d\end{array}\right)$ satisfies $\operatorname{deg}(b) \neq 0, \operatorname{deg}(a+b \phi) \neq 0$, and $\operatorname{deg}(c+d \phi) \neq 0$, then we have

$$
N(g, L)=\operatorname{deg}(a+b \phi)^{1 / 2} \cdot q(g)^{1 / 2} \cdot \frac{\operatorname{rk} V_{g(\phi)}}{\operatorname{rk} V_{\phi}},
$$

where $q(g)$ is given by (2.1.13), $\mathrm{rk} V_{\phi}$ is given by (2.1.9), and

$$
\lambda(g, \Gamma(\phi))=-i\left(b^{-1} a+\phi\right) .
$$

Proof

(i) Let us extend $L$ and $L(g)$ to Lagrangian pairs $(L, \alpha)$ and $(L(g), \beta)$. By [31, Theorem 3.2.11] applied to the Lagrangian correspondence $(L(g), \beta)$ and to $(L, \alpha)$ viewed as a Lagrangian correspondence from 0 to $X_{A}$, we obtain

$$
\Phi_{L(g), \beta}\left(S_{L, \alpha}\right)=S_{L(g) \circ L, \beta \circ \alpha}[i]
$$

for some $i \in \mathbb{Z}$. As in [31, Theorem 3.2.14] one can check that $i$ does not depend on $\alpha$ and $\beta$. Next, we have to relate the composed Lagrangian correspondence $S_{L(g) \circ L, \beta \circ \alpha}$ with $S(g L)$. Here we use the definition of the composition of 
Lagrangian correspondences from [31, Section 3]. Note that the result is a generalized Lagrangian correspondence in the sense of [31, Definition 3.1.1] We are going to apply [31, Proposition 2.4.7(ii)] to the generalized Lagrangian $Z:=$ $L(g) \circ L \stackrel{j}{\longrightarrow} X_{A}$. Note that $Z \subset L(g) \subset X_{A} \times X_{A}$ is the preimage of $L$ under the first projection $p_{1}: L(g) \rightarrow X_{A}$, and the homomorphism $j: Z \rightarrow X_{A}$ is induced by the second projection $p_{2}: L(g) \rightarrow X_{A}$. By [31, Proposition 2.4.7(ii)], we have

$$
S_{Z, \beta \circ \alpha} \equiv n^{1 / 2} \cdot\left|\pi_{0}(Z)\right|^{1 / 2} \cdot S\left(j\left(Z_{0}\right)\right)
$$

in $\overline{\mathrm{SH}}^{\mathrm{LI}}(A \times A)$, where $n=\left|\pi_{0}(j(Z))\right|$. (Here $Z_{0}$ is the connected component of 0 in $Z$.) By definition, we have $j\left(Z_{0}\right)=g L$. Thus, we deduce (2.4.1) with

$$
N(g, L)=\left|\pi_{0}(Z)\right|^{1 / 2} \cdot n^{1 / 2} .
$$

Also, by [31, (2.4.12)], we have $n=\operatorname{deg}\left(Z_{0} \rightarrow j\left(Z_{0}\right)\right)$.

(ii) Now assume that $L=\Gamma(\phi)$, and assume that $g(\phi)$ is defined and is an isogeny. Note that for sufficiently divisible $N$ we have an isogeny

$$
A \rightarrow Z_{0}: x \mapsto(N x, N \phi x, N(a+b \phi) x, N(c+d \phi) x) \in L(g) \subset X_{A} \times X_{A} .
$$

In particular, both projections from $Z_{0}$ to $A$ are isogenies. Let us consider the commutative diagram of isogenies

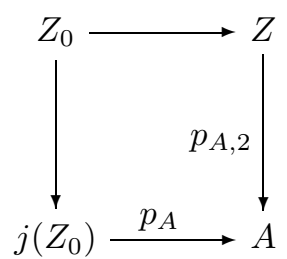

where $p_{A, 2}$ is the composition $Z \rightarrow L(g) \stackrel{p_{2}}{\longrightarrow} X_{A} \stackrel{p_{A}}{\longrightarrow} A$. Considering the degrees we obtain

$$
\operatorname{deg}\left(p_{A, 2}: Z \rightarrow A\right)=\left|\pi_{0}(Z)\right| \cdot \operatorname{deg}\left(p_{A, 2} \mid Z_{0}\right)=\left|\pi_{0}(Z)\right| \cdot \operatorname{deg}\left(j\left(Z_{0}\right) \rightarrow A\right) \cdot n .
$$

Recall that $j\left(Z_{0}\right)=g L$, so we get that

$$
N(g, L)=\frac{\operatorname{deg}\left(p_{A, 2}: Z \rightarrow A\right)^{1 / 2}}{\operatorname{deg}(g L \rightarrow A)^{1 / 2}} .
$$

Now let us consider the projection $p_{A, 1}: Z \rightarrow L(g) \stackrel{p_{1}}{\longrightarrow} X_{A} \stackrel{p_{A}}{\longrightarrow} A$. Using the isogeny (2.4.4) we see that

$$
\left.N p_{A, 2}\right|_{Z_{0}}=\left.N(a+b \phi) p_{A, 1}\right|_{Z_{0}} .
$$

Hence,

$$
\frac{\operatorname{deg}\left(p_{A, 2}: Z \rightarrow A\right)}{\operatorname{deg}\left(p_{A, 1}: Z \rightarrow A\right)}=\frac{\operatorname{deg}\left(\left.p_{A, 2}\right|_{Z_{0}}\right)}{\operatorname{deg}\left(\left.p_{A, 1}\right|_{Z_{0}}\right)}=\operatorname{deg}(a+b \phi) .
$$

Note that $p_{A, 1}$ factors through the projection $Z \rightarrow L$ and we have a Cartesian square 


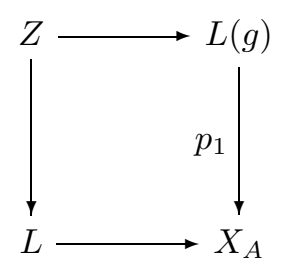

which shows that $\operatorname{deg}(Z \rightarrow L)=\operatorname{deg}\left(p_{1}: L(g) \rightarrow X_{A}\right)=q(L(g))$. Thus,

$$
\operatorname{deg}\left(p_{A, 1}: Z \rightarrow A\right)=q(L(g)) \cdot \operatorname{deg}(L \rightarrow A)
$$

and (2.4.5) can be rewritten as

$$
\operatorname{deg}\left(p_{A, 2}: Z \rightarrow A\right)=\operatorname{deg}(a+b \phi) \cdot q(L(g)) \cdot \operatorname{deg}(L \rightarrow A) .
$$

Therefore,

$$
N(g, L)=\operatorname{deg}(a+b \phi)^{1 / 2} \cdot q(L(g))^{1 / 2} \frac{\operatorname{deg}(L \rightarrow A)^{1 / 2}}{\operatorname{deg}(g L \rightarrow A)^{1 / 2}} .
$$

Recalling that $\operatorname{rk} S(L)=\operatorname{deg}(L \rightarrow A)^{1 / 2}$ we obtain (2.4.2).

Finally, to compute $\lambda(g, \Gamma(\phi))$ we apply [31, Proposition 3.2.9]. Namely, we have to consider the fibered product $\Gamma(\phi) \times{ }_{A} L(g)$ where we use the first projection $L(g) \rightarrow A$. Note that we have an isogeny

$$
\begin{aligned}
A \times \hat{A} & \rightarrow\left(\Gamma(\phi) \times{ }_{A} L(g)\right)_{0}:(x, \xi) \\
& \mapsto((N x, N \phi x),(N x, N \xi, N(a x+b \xi), N(c x+d \xi))),
\end{aligned}
$$

where $N$ is sufficiently divisible. Next, we set

$$
F=\operatorname{ker}\left(\Gamma(\phi) \times_{A} L(g) \stackrel{\gamma}{\longrightarrow} A\right),
$$

where $\gamma$ is induced by the projection to $L(g)$ followed by $L(g) \stackrel{p_{2}}{\longrightarrow} X_{A} \rightarrow A$. Note that the composition of $\gamma$ with the isogeny (2.4.6) is given by $(x, \xi) \mapsto N(a x+b \xi)$. Hence, we have an isogeny

$$
A \rightarrow F_{0}: x \mapsto\left((N x, N \phi x),\left(N x,-N b^{-1} a x, 0, N\left(c x-d b^{-1} a x\right)\right)\right) .
$$

By [31, Proposition 3.2.9], we have

$$
\lambda(g, \Gamma(\phi))=-i\left(g_{0} \circ f_{0}^{-1}\right),
$$

where $f_{0}: F_{0} \rightarrow A$ is the natural projection and, for $(l, m) \in F_{0} \subset \Gamma(\phi) \times L(g)$,

$$
g_{0}(l, m)=p_{\hat{A}}(l)-p_{\hat{A}, 1}(m),
$$

where $p_{\hat{A}}: \Gamma(\phi) \rightarrow \hat{A}$ is the natural projection and $p_{\hat{A}, 1}$ is the composition $L(g) \stackrel{p_{1}}{\longrightarrow} X_{A} \rightarrow \hat{A}$. Thus, the compositions of $f_{0}$ and $g_{0}$ with the isogeny (2.4.7) are $x \mapsto N x$ and $x \mapsto N\left(\phi+b^{-1} a x\right)$, respectively. Hence,

$$
g_{0} \circ f_{0}^{-1}=\phi+b^{-1} a
$$

as required. 
Let us set

$$
\overline{\mathrm{SH}}^{\mathrm{LI}}(A)_{\mathbb{R}}=\overline{\mathrm{SH}}^{\mathrm{LI}}(A) \times \mathbb{R}_{>0} / \mathbb{N}^{*},
$$

where $n \in \mathbb{N}^{*}$ acts by $(F, r) \mapsto\left(n F, n^{-1} r\right)$. Then the bijection of Proposition 2.1.2 extends to a bijection of $\mathbb{R}_{>0} \times \mathbb{Z}$ sets

$$
\mathbf{L G}(\mathbb{Q}) \times \mathbb{R}_{>0} \times \mathbb{Z} \stackrel{\sim}{\longrightarrow} \overline{\mathrm{SH}}^{\mathrm{LI}}(A)_{\mathbb{R}} .
$$

\section{COROLLARY 2.4.2}

There is an action of $\widetilde{\mathbf{U}(\mathbb{Q})}$ on $\overline{\mathrm{SH}}^{\mathrm{LI}}(A)_{\mathbb{R}}$, commuting with the $\mathbb{R}_{>0}$-action, such that $(g, n)$ acts by

$$
F \mapsto q(L(g))^{-1 / 2} \cdot \Phi_{g}(F)[n] .
$$

For $g_{1}, g_{2} \in \mathbf{U}(\mathbb{Q})$ and $L \in \mathbf{L G}(\mathbb{Q})$ we have

$$
\lambda\left(g_{1}, g_{2}(L)\right)+\lambda\left(g_{2}, L\right)=\lambda\left(g_{1}, g_{2}\right)+\lambda\left(g_{1} g_{2}, L\right) .
$$

Also, the maps $\Phi_{g}$ induce an action of $\widetilde{\mathbf{U}(\mathbb{Q})}$ on $\overline{\mathrm{SH}}^{\mathrm{LI}}(A) / \mathbb{N}^{*} \simeq \mathbf{L G}(\mathbb{Q}) \times \mathbb{Z}$.

Note that the natural maps

$$
\begin{aligned}
\overline{\mathrm{SH}}^{\mathrm{LI}}(A) & \rightarrow \mathcal{N}(A) \otimes \mathbb{Q} / \mathbb{Q}_{>0}, \\
\overline{\mathrm{SH}}^{\mathrm{LI}}(A)_{\mathbb{R}} & \rightarrow \mathcal{N}(A) \otimes \mathbb{R}
\end{aligned}
$$

associating with an LI sheaf $F$ its class $[F]$ in $\mathcal{N}(A)$ are $\widetilde{\mathbf{U}(\mathbb{Q})}$-equivariant, where the action on $\mathcal{N}(A) \otimes \mathbb{R}$ is given by $\hat{\rho}$ (see $(2.1 .17)$ ).

\subsection{Action of $\operatorname{Spin}$ on $\mathcal{N}(A)_{\mathbb{R}}$}

We are going to define an algebraic action of Spin on $\mathcal{N}(A)_{\mathbb{R}}$ inducing the homomorphism $\hat{\rho}$ on $\mathbf{U}(\mathbb{Q})^{\text {spin }} \subset \operatorname{Spin}(\mathbb{R})$. The idea is to use the algebraicity of the corresponding projective representation and of the action of an open subset on a fixed nonzero vector. We will need the following simple result.

\section{LEMMA 2.5.1}

Let $V$ be a vector space over a field $F$, let $X$ be a scheme (resp., a set), and let $\bar{f}: X \rightarrow \mathbb{P}(V), \bar{g}: X \rightarrow \mathbb{P}(V)$, and $\bar{h}: X \rightarrow \mathbb{P}(V)$ be regular morphisms (resp., maps to the set of $F$-points) such that the lines $\bar{f}(x), \bar{g}(x)$, and $\bar{h}(x)$ are all distinct and $\bar{h}(x) \subset \operatorname{span}(\bar{f}(x), \bar{g}(x))$ for each $x \in X$. Suppose we have a lifting of $\bar{f}$ to a regular morphism (resp., map to the set of F-points) $f: X \rightarrow V-\{0\}$. Then there exist unique liftings of $\bar{g}$ and $\bar{h}$ to regular morphisms (resp., maps to the set of F-points) $g, h: X \rightarrow V-\{0\}$ such that $h=f+g$.

Proof

Consider a subvariety

$$
Y \subset(V-\{0\}) \times(V-\{0\}) \times(V-\{0\})
$$


consisting of $\left(v_{1}, v_{2}, v_{1}+v_{2}\right)$ such that $v_{1}$ and $v_{2}$ are linearly independent, and consider a subvariety

$$
\bar{Y} \subset(V-\{0\}) \times \mathbb{P}(V) \times \mathbb{P}(V)
$$

consisting of $\left(v, L, L^{\prime}\right)$ such that $v \notin L, v \notin L^{\prime}, L \neq L^{\prime}$, and $v \in L+L^{\prime}$. Then the natural projection $p: Y \rightarrow \bar{Y}$ is an isomorphism. We have a regular morphism (resp., map to the set of $F$-points) $(f, \bar{g}, \bar{h}): X \rightarrow \bar{Y}$. Now the components of the corresponding map $X \rightarrow Y$ give the required liftings.

\section{LEMMA 2.5.2}

For a symmetric isogeny $\phi \in \mathrm{NS}^{0}(A, \mathbb{Q})$ we have

$$
\frac{\left[V_{\phi}\right]}{\mathrm{rk} V_{\phi}}=\ell(\phi) \in \mathcal{N}(A) \otimes \mathbb{Q},
$$

where $V_{\phi}$ is the semihomogeneous vector bundle (2.1.4) and $\ell: \mathrm{NS}(A) \otimes \mathbb{Q} \rightarrow$ $\mathcal{N}(A) \otimes \mathbb{Q}$ is the polynomial map (1.1.1).

\section{Proof}

Since $\operatorname{rk} \ell(\phi)=1$, it suffices to check the required identity up to proportionality. Recall that if $(L=\Gamma(\phi), \alpha)$ is a Lagrangian pair, then the line in $\mathcal{N}(A) \otimes \mathbb{Q}$ corresponding to $V_{\phi}$ is spanned by the class of $p_{A *}(\mathcal{L})$, where $p_{A}: L \rightarrow A$ is the projection and $\mathcal{L}=\left.\alpha^{-1} \otimes \mathcal{P}\right|_{L}$ (see (2.1.7)). Also, by the definition of a Lagrangian pair,

$$
\Lambda(\alpha)_{l_{1}, l_{2}} \simeq \mathcal{P}_{p_{A}\left(l_{1}\right), p_{\hat{A}}\left(l_{2}\right)}
$$

so $\phi_{\mathcal{L}}: L \rightarrow \hat{L}$ is given as

$$
\phi_{\mathcal{L}}=\widehat{p_{A}} \circ p_{\hat{A}}=\widehat{p_{\hat{A}}} \circ p_{A},
$$

where $p_{\hat{A}}: L \rightarrow \hat{A}$ is the projection. Note that for sufficiently divisible $N$ we have an isogeny

$$
i: A \rightarrow L: x \mapsto(N x, N \phi x)
$$

and the classes of $p_{A *}(\mathcal{L})$ and $[N]_{*}\left(i^{*} \mathcal{L}\right)$ in $\mathcal{N}(A) \otimes \mathbb{Q}$ are proportional. We have

$$
\phi_{i^{*}(\mathcal{L})}=\hat{i} \circ \phi_{\left.\alpha^{-1} \otimes \mathcal{P}\right|_{L}} \circ i=\widehat{p_{A} \circ i} \circ p_{\hat{A} \circ i}=N^{2} \phi .
$$

Thus, the class $\left[i^{*}(\mathcal{L})\right] \in \mathcal{N}(A) \otimes \mathbb{Q}$ is proportional to $\ell\left(N^{2} \phi\right)=[N]^{*} \ell(\phi)$. Hence, the class of $[N]_{*}\left(i^{*} \mathcal{L}\right)$ is proportional to

$$
[N]_{*}[N]^{*} \ell(\phi)=N^{2 g} \ell(\phi)
$$

as required.

THEOREM 2.5.3

The homomorphism $\hat{\rho}: \mathbf{U}(\mathbb{Q})^{\text {spin }} \rightarrow \mathrm{GL}(\mathcal{N}(A) \otimes \mathbb{R})$ (see (2.1.17)) extends to an algebraic homomorphism

$$
\hat{\rho}: \operatorname{Spin} \rightarrow \mathrm{GL}\left(\mathcal{N}(A)_{\mathbb{R}}\right)
$$


defined over $\mathbb{R}$. For $(g, f) \in \operatorname{Spin}(\mathbb{C})$ and $\phi \in \mathrm{NS}^{0}(A, \mathbb{C})$ such that $g(\phi)$ is defined and belongs to $\operatorname{NS}^{0}(A, \mathbb{C})$, we have

$$
\hat{\rho}(g, f)(\ell(\phi))=f(\phi) \cdot \ell(g(\phi)) .
$$

\section{Proof}

First, we observe that Theorem 2.4.1 implies (2.5.1) in the case when $(g, f) \in$ $\mathbf{U}(\mathbb{Q})^{\text {spin }} \subset \operatorname{Spin}(\mathbb{R})$ with $g \in \mathbf{U}^{0}(\mathbb{Q})$ and $\phi \in \operatorname{NS}^{0}(A, \mathbb{Q})$ is such that $g(\phi)$ is defined and belongs to $\operatorname{NS}^{0}(A, \mathbb{Q})$. Indeed, from (2.4.1), (2.4.3), (2.4.2), and Lemma 2.5.2 we obtain in this case

$$
\begin{aligned}
\hat{\rho}(g)(\ell(\phi)) & =(-1)^{i\left(b^{-1} a+\phi\right)}|\operatorname{deg}(a+b \phi)|^{1 / 2} \cdot \ell(g(\phi)) \\
& =\operatorname{deg}(b)^{1 / 2} \cdot \chi\left(b^{-1} a+\phi\right) \cdot \ell(g(\phi)) .
\end{aligned}
$$

Thus, our claim holds for

$$
\bar{\iota}(g, 0)=\left(g, \operatorname{deg}(b)^{1 / 2} \cdot \chi\left(b^{-1} a+\phi\right)\right) .
$$

It remains to note that both sides of $(2.5 .1)$ change sign when $(g, f)$ gets multiplied by $-1 \in\{ \pm 1\} \subset$ Spin.

By [26, Theorem 5.1], there is an algebraic homomorphism $\mathbf{U} \rightarrow$ $\operatorname{PGL}\left(\mathcal{N}(A)_{\mathbb{Q}}\right)$ sending $g \in \mathbf{U}(\mathbb{Q})$ to $\rho(g) \bmod \mathbb{Q}^{*}$. Let us denote the corresponding action of $\mathbf{U}$ on $\mathbb{P}\left(\mathcal{N}(A)_{\mathbb{R}}\right)$ by

$$
\bar{\kappa}: \mathbf{U} \times \mathbb{P}\left(\mathcal{N}(A)_{\mathbb{R}}\right) \rightarrow \mathbb{P}\left(\mathcal{N}(A)_{\mathbb{R}}\right) .
$$

We also have a map

$$
\kappa^{\mathbb{Q}}: \mathbf{U}(\mathbb{Q})^{\operatorname{spin}} \times \mathcal{N}(A) \otimes \mathbb{R} \rightarrow \mathcal{N}(A) \otimes \mathbb{R}:(\widetilde{g}, \mathbf{v}) \mapsto \hat{\rho}(\widetilde{g})(\mathbf{v})
$$

inducing the restriction of $\bar{\kappa}(\mathbb{R})$ to $\mathbf{U}(\mathbb{Q})^{\text {spin }} \times \mathbb{P}(\mathcal{N}(A) \otimes \mathbb{R})$. We are going to extend $\kappa^{\mathbb{Q}}$ to an algebraic morphism using the density of $\mathbf{U}(\mathbb{Q})$ in $\mathbf{U}$ (see Lemma 1.3.4).

Note that for a fixed isogeny $\phi$ the right-hand side of (2.5.1) extends to a regular morphism (defined over $\mathbb{Q})$

$$
\kappa_{\ell(\phi)}: \pi^{-1}(V) \rightarrow \mathcal{N}(A)_{\mathbb{R}}
$$

where $V \subset \mathbf{U}^{0} \subset \mathbf{U}$ is an open subset of $g \in \mathbf{U}^{0}$ such that $g(\phi)$ is defined and is an isogeny. Furthermore, as we have seen in the beginning of the proof, the corresponding map on $\pi^{-1}(V(\mathbb{Q}))$ coincides with the restriction of $\kappa^{\mathbb{Q}}$ to $\pi^{-1}(V(\mathbb{Q})) \times\{\ell(\phi)\}$. In particular, the map

$$
\bar{\kappa}_{\ell(\phi)}: \pi^{-1}(V) \rightarrow \mathbb{P}\left(\mathcal{N}(A)_{\mathbb{R}}\right)
$$

obtained from $\kappa_{\ell(\phi)}$ is the composition of the projection to $V$ with the restriction of $\bar{\kappa}$ to $\mathbf{U} \times\{\langle\ell(\phi)\rangle\}$ (since we know this on the dense subset $V(\mathbb{Q})$ ).

Now if $\mathbf{v} \in \mathcal{N}(A) \otimes \mathbb{Q}$ is any vector, linearly independent with $\ell(\phi)$, then by Lemma 2.5.1, we obtain unique liftings

$$
\kappa_{\mathbf{v}}, \kappa_{\ell(\phi)+\mathbf{v}}: \pi^{-1}(V) \rightarrow \mathcal{N}(A)_{\mathbb{R}}
$$


of the restrictions of $\bar{\kappa}$ to $\pi^{-1}(V) \times\{\langle\mathbf{v}\rangle\}$ and $\pi^{-1}(V) \times\{\langle\ell(\phi)+\mathbf{v}\rangle\}$ such that

$$
\kappa_{\ell(\phi)+\mathbf{v}}(\widetilde{g})=\kappa_{\ell(\phi)}(\widetilde{g})+\kappa_{\mathbf{v}}(\widetilde{g})
$$

Furthermore, the set-theoretic part of Lemma 2.5.1 implies that the maps (2.5.2) induce the corresponding restrictions of $\kappa^{\mathbb{Q}}$ on $\pi^{-1}(V(\mathbb{Q}))$.

Thus, if we consider a basis of $\mathcal{N}(A) \otimes \mathbb{Q}$ of the form $\left(\ell(\phi), \mathbf{v}_{1}, \ldots, \mathbf{v}_{n}\right)$, then combining the maps $\kappa_{\mathbf{v}_{i}}$ constructed above we get a regular morphism

$$
\hat{\rho}_{V}: \pi^{-1}(V) \rightarrow \operatorname{GL}\left(\mathcal{N}(A)_{\mathbb{R}}\right)
$$

inducing $\hat{\rho}$ on $\pi^{-1}(V(\mathbb{Q}))$. We can cover Spin with open subsets of the form $\pi^{-1}(V) \widetilde{g}$ with $\widetilde{g} \in \mathbf{U}(\mathbb{Q})^{\text {spin }}$ and define regular morphisms $\pi^{-1}(V) \widetilde{g} \rightarrow$ $\operatorname{GL}\left(\mathcal{N}(A)_{\mathbb{R}}\right)$ by sending $\widetilde{h} \widetilde{g}$ to $\hat{\rho}_{V}(\widetilde{h}) \hat{\rho}(\widetilde{g})$. Using the density of $\mathbf{U}(\mathbb{Q})^{\text {spin }}$ in Spin, one easily checks that these maps glue into the required algebraic homomorphism $\pi^{-1}(V) \rightarrow \operatorname{GL}\left(\mathcal{N}(A)_{\mathbb{R}}\right)$

Consider the action of $\operatorname{Spin}(\mathbb{R})$ on the trivial $\mathbb{C}^{*}$-bundle $D_{A} \times \mathbb{C}^{*}$ over the domain $D_{A}$ given by

$$
(g, f) \cdot(\omega, z)=(g(\omega), f(\omega) \cdot z),
$$

where $(g, f) \in \operatorname{Spin}(\mathbb{R}), \omega \in D_{A}$, and $z \in \mathbb{C}^{*}$. The map $\ell: D \rightarrow \mathcal{N}(A) \otimes \mathbb{C}$ (see (1.1.1)) extends to a $\mathbb{C}^{*}$-equivariant map

$$
\ell: D_{A} \times \mathbb{C}^{*} \rightarrow \mathcal{N}(A) \otimes \mathbb{C}:(\omega, z) \mapsto z \cdot \ell(\omega) .
$$

From the identity (2.5.1) we immediately get the following result.

COROLLARY 2.5.4

The map (2.5.3) is $\operatorname{Spin}(\mathbb{R})$-equivariant.

PROPOSITION 2.5.5

For any $x, y \in \mathcal{N}(A) \otimes \mathbb{C}$ and any $\widetilde{g} \in \operatorname{Spin}(\mathbb{C})$ one has

$$
\chi(\hat{\rho}(\widetilde{g})(x), \hat{\rho}(\widetilde{g})(y))=\chi(x, y) .
$$

\section{Proof}

Note that the left-hand side of (2.5.4) depends only on the image of $\widetilde{g}$ in $\mathbf{U}(\mathbb{C})$. Let us first consider the case when this image is an element $g \in \mathbf{U}(\mathbb{Q})$. Consider the functor $\Phi=\Phi_{L(g), \alpha}: D^{b}(A) \rightarrow D^{b}(A)$ associated with some Lagrangian correspondence $(L(g), \alpha)$ extending $g$, so that $\Phi$ represents the $\mathbf{H}$-equivalence class of $\Phi_{g}$. Let $\Psi$ be the right adjoint functor to $\Phi$. By [31, Proposition 3.2.7], $\Psi$ differs by a shift from the LI functor associated with some Lagrangian correspondence extending $L\left(g^{-1}\right)$. Applying (2.1.11) and (2.1.12) for $g_{1}=g$ and $g_{2}=g^{-1}$ we obtain

$$
\Psi \circ \Phi \equiv N \cdot \mathrm{Id}
$$


where $N=q(g)^{1 / 2} q\left(g^{-1}\right)^{1 / 2}$. Since for $F, G \in D^{b}(A)$ we have an isomorphism

$$
\operatorname{Hom}^{*}(\Phi(F), \Phi(G))=\operatorname{Hom}^{*}(F, \Psi \Phi(G)),
$$

we deduce the equality

$$
\chi(\hat{\rho}(g)([F]), \hat{\rho}(g)([G]))=\frac{q\left(g^{-1}\right)^{1 / 2}}{q(g)^{1 / 2}} \cdot \chi([F],[G]) .
$$

Since $\mathbf{U}(\mathbb{Q})$ is dense in $\mathbf{U}$ (see Lemma 1.3.4), there exists an algebraic character $\varpi: \mathbf{U} \rightarrow \mathbb{G}_{m}$ such that

$$
\chi(\hat{\rho}(g)(x), \hat{\rho}(g)(y))=\varpi(g) \cdot \chi(x, y)
$$

for any $g \in \mathbf{U}(\mathbb{C})$. The character $\varpi$ restricts trivially to the semisimple subgroup $S \mathbf{U} \subset \mathbf{U}$. Thus, by Lemma 1.3.1(i), it remains to show the triviality of its restriction to $\mathbf{Z}$. In fact, we will show directly that $\varpi(t)=1$ for any $t=\left(\begin{array}{cc}a^{-1} & 0 \\ 0 & \hat{a}\end{array}\right) \in \mathbf{T}(\mathbb{Q})$, where $a \in(\operatorname{End}(A) \otimes \mathbb{Q})^{*}$. Note that this implies that $\left.\varpi\right|_{\mathbf{T}}=1$ since $\mathbf{T}(\mathbb{Q})$ is dense in $\mathbf{T}$. It suffices to consider the case when $a \in \operatorname{End}(A)$. Then the correspondence $L(t) \subset X_{A} \times X_{A}$ is the image of the embedding

$$
A \times \hat{A} \rightarrow X_{A} \times X_{A}:(x, \xi) \mapsto(a x, \xi, x, \hat{a}(\xi)) .
$$

Hence, in this case $q(t)=\operatorname{deg}(a)$ and $q\left(t^{-1}\right)=\operatorname{deg}(\hat{a})=\operatorname{deg}(a)$, and our assertion follows from (2.5.5).

\section{COROLLARY 2.5.6}

For $\widetilde{g}=\left(g, f_{g}\right) \in \operatorname{Spin}(\mathbb{C}), \omega \in D_{A}$, and $x \in \mathcal{N}(A) \otimes \mathbb{C}$ one has

$$
\chi\left(\ell(\omega), \hat{\rho}(\widetilde{g})^{-1}(x)\right)=f_{g}(\omega) \cdot \chi(\ell(g(\omega)), x) .
$$

Proof

Indeed, we have

$$
\chi\left(\ell(\omega), \hat{\rho}(\widetilde{g})^{-1}(x)\right)=\chi(\hat{\rho}(\widetilde{g})(\ell(\omega)), x)=f_{g}(\omega) \cdot \chi(\ell(g(\omega)), x) .
$$

\section{COROLLARY 2.5.7}

For any $g \in \mathbf{U}(\mathbb{Q})$ one has $q(g)=q\left(g^{-1}\right)$.

\section{EXAMPLES 2.5.8}

(1) If $A$ is an abelian variety of dimension $n$ over $\mathbb{C}$ without complex multiplication, then we have $\operatorname{NS}(A)=\mathbb{Z} \cdot H$, where $H$ is an ample generator, and so $\tau \mapsto \tau \phi_{H}$ gives an identification $\mathfrak{H} \rightarrow D_{A}$, where $\mathfrak{H}$ is the upper half-plane. The group $\mathbf{U}(\mathbb{R})$ can be identified with $\operatorname{SL}(2, \mathbb{R})$ with the action on $\mathfrak{H} \simeq D_{A}$ given by fractional-linear transformations (1.4.3). Since $\Delta(g)\left(\tau \cdot \phi_{H}\right)=(a+b \tau)^{2 n}$, we have a natural splitting

$$
\mathrm{SL}(2, \mathbb{R}) \rightarrow \operatorname{Spin}_{X_{A}}(\mathbb{R}): g \mapsto\left(g,(a+b \tau)^{n}\right) .
$$

Furthermore, if $A$ is generic, then $\mathcal{N}(A) \otimes \mathbb{Q}$ can be identified with the $(g+1)$ dimensional subspace in $H^{*}(A, \mathbb{Q})$ spanned by the classes $H^{i}, i=0, \ldots, g$, and 
(2.5.1) shows that $\mathrm{SL}(2, \mathbb{R})$ acts on $\mathcal{N}(A) \otimes \mathbb{R}$ as on the standard $(g+1)$ dimensional irreducible representation. Assume in addition that $\phi_{H}$ is a principal polarization of $A$. Then we can index simple semihomogeneous vector bundles by rational numbers. Namely, for coprime integers $(r, d)$ with $r>0$ we set

$$
V_{r, d}=V_{(d / r) \phi_{H}} .
$$

From (2.1.9) we get in this case that $\mathrm{rk} V_{r, d}=r^{n}$ (see also [19, Remark 7.13], [27, Chapter 12, Exercise 2]). Hence, by Lemma 2.5.2,

$$
\operatorname{ch}\left(V_{r, d}\right)=\sum_{i=0}^{n} r^{n-i} d^{i} \cdot \frac{H^{i}}{i !} \in H^{*}(A, \mathbb{Z}) .
$$

Note that for $r=0, d=1$ this formula gives $\operatorname{ch}\left(\mathcal{O}_{x}\right)$. Using the HirzebruchRiemann-Roch formula we get the following relations for the form $\chi$ on $\mathcal{N}(A) \otimes \mathbb{Q}:$

$$
\begin{aligned}
\chi\left(H^{i}, H^{n-i}\right) & =(-1)^{i} n !, \\
\chi\left(\ell\left(\tau \phi_{H}\right),\left[V_{r, d}\right]\right) & =(d-r \tau)^{n} .
\end{aligned}
$$

(2) Continuing the previous example, assume in addition that $n=\operatorname{dim} A=3$ (keeping the assumptions that $A$ is principally polarized and generic). Then $A$ is the Jacobian of a curve, so $H^{2} / 2$ is an algebraic class. We claim that the image of the Chern character ch $: K_{0}(A) \rightarrow H^{*}(A, \mathbb{Q})$ contains the $\mathbb{Z}$-submodule $K \subset H^{*}(A, \mathbb{Q})$ spanned by $\left(H^{i} / i !\right)_{0 \leq i \leq n}$. Indeed, the Chern characters of the structure sheaves of a point and of the curve span the submodule $\mathbb{Z} H^{2} / 2+$ $\mathbb{Z} H^{3} / 6$. Together with $\operatorname{ch}\left(\mathcal{O}_{A}\right)=1$ and $\operatorname{ch}(\mathcal{O}(H))=\exp (H)$ these classes span the whole $\mathbb{Z}$-submodule $K$. On the other hand, using the above formula we see that for $n \geq 3$ the images of the Chern characters of LI sheaves (which are all $\mathbf{H}$ equivalent to either $V_{d, r}$ or $\mathcal{O}_{x}$ ) span a proper $\mathbb{Z}$-submodule in $K$. In particular, the LI objects do not generate $D^{b}(A)$ in this case.

(3) If $A$ is an elliptic curve over $\mathbb{C}$ with complex multiplication, then we have an isomorphism

$$
\mathbf{U}(\mathbb{R}) \simeq \mathrm{SL}(2, \mathbb{R}) \times \mathbf{U}(1) /\{ \pm 1\},
$$

where $\{ \pm 1\}$ is embedded into the product diagonally. Also, $D_{A}=\mathfrak{H}$, the upper half-plane, and $\mathbf{U}(\mathbb{R})$ acts on $D_{A}$ through the projection to $\operatorname{SL}(2, \mathbb{R}) /\{ \pm 1\}$. The spin covering $\operatorname{Spin}_{X_{A}}(\mathbb{R}) \rightarrow \mathbf{U}(\mathbb{R})$ in this case can be identified with the natural covering

$$
\mathrm{SL}(2, \mathbb{R}) \times \mathbf{U}(1) \rightarrow \mathbf{U}(\mathbb{R})
$$

\section{Action on stability spaces}

\subsection{Induced $t$-structures and stabilities}

We refer to [6] for notions related to stability conditions on triangulated categories. All $t$-structures considered below are assumed to be bounded and nondegenerate (see [3]). All stabilities are assumed to be locally finite and numerical. 
We say that a $t$-structure (resp., a slicing or a stability condition) on $D^{b}(A)$ is $\mathbf{H}$-invariant if it is invariant under any functor $T_{(x, \xi)}$ with $(x, \xi) \in A \times \hat{A}$ (see (2.1.3)), that is, under translations and tensoring by $\operatorname{Pic}^{0}(A)$. Note that, by [29, Corollary 3.5.2], every full stability condition is $\mathbf{H}$-invariant.

The general construction of the induced $t$-structures (resp., stability conditions) from [29] and [18] specializes to the following result on inducing $\mathbf{H}$-invariant $t$-structures.

\section{PROPOSITION 3.1.1}

Let $A$ and $B$ be abelian varieties of the same dimension, and let $\Phi: D^{b}(A) \rightarrow$ $D^{b}(B)$ be the LI functor associated with a Lagrangian correspondence $(L, \alpha)$ from $X_{A}$ to $X_{B}$ such that the projections $L \rightarrow X_{A}$ and $L \rightarrow X_{B}$ are surjective, with the right adjoint functor $\Phi^{\prime}: D^{b}(B) \rightarrow D^{b}(A)$. Also, let $\left(D^{\leq 0}, D^{\geq 0}\right)$ be an $\mathbf{H}$-invariant $t$-structure on $D^{b}(A)$. Then there is a unique $\mathbf{H}$-invariant $t$-structure $\left({ }^{\Phi} D^{\leq 0},{ }^{\Phi} D^{\geq 0}\right)$ on $D^{b}(B)$ such that

$$
\Phi\left(D^{[a, b]}\right) \subset{ }^{\Phi} D^{[a, b]} .
$$

It is given by

$$
{ }^{\Phi} D^{[a, b]}=\left\{F \in D^{b}(B) \mid \Phi^{\prime}(F) \in D^{[a, b]}\right\} .
$$

Similarly, if $(P(t))_{t \in \mathbb{R}}$ is an $\mathbf{H}$-invariant slicing on $D^{b}(A)$, then there is a unique $\mathbf{H}$-invariant slicing $\left({ }^{\Phi} P(t)\right)_{t \in \mathbb{R}}$ on $D^{b}(B)$ such that $\Phi(P(t)) \subset{ }^{\Phi} P(t)$ for any $t \in \mathbb{R}$. We have ${ }^{\Phi} P(t)=\left(\Phi^{\prime}\right)^{-1}(P(t))$.

\section{Proof}

First, we observe that, by [31, Proposition 3.2.7], $\Phi^{\prime}$ differs by a shift from the LI functor associated with the transposed correspondence $\left(\sigma(L), \alpha^{-1}\right)$, where $\sigma: X_{A} \times X_{B} \rightarrow X_{B} \times X_{A}$ is the permutation of factors. Hence, the same argument as in [31, Lemma 3.3.3] shows that both compositions $\Phi^{\prime} \circ \Phi$ and $\Phi \circ \Phi^{\prime}$ are obtained by consecutive extensions from functors $T_{(x, \xi)}$, one of which is the identity functor.

The fact that (3.1.2) defines a $t$-structure follows from [29, Theorem 2.1.2] once we check that in our situation $\Phi^{\prime} \circ \Phi$ is $t$-exact with respect to the original $t$-structure and $\left(\Phi \circ \Phi^{\prime}\right)(F)=0$ implies that $F=0$. Indeed, the former follows from the $\mathbf{H}$-invariance of our $t$-structure. To check the latter property it suffices to consider the case when $F$ is a coherent sheaf. We observe that the right adjoint functor to $\Phi \circ \Phi^{\prime}$ sends a structure sheaf of a point $\mathcal{O}_{x}$ to a sheaf $K_{x}$ supported on a finite number of points, including $x$. Hence, if $\left(\Phi \circ \Phi^{\prime}\right)(F)=0$, then $\operatorname{Hom}\left(F, K_{x}\right)=0$ for all $x \in B$, which implies that $F=0$.

The inclusion (3.1.1) follows from the $\mathbf{H}$-invariance of the original $t$-structure and from the form of $\Phi^{\prime} \circ \Phi$. The fact that the new $t$-structure is $\mathbf{H}$-invariant follows from the $\mathbf{H}$-intertwining property of LI functors (see (2.1.5) and [31, Lemma 3.2.4]). Now suppose that $\left({ }^{\Phi} D_{1} \leq 0,{ }^{\Phi} D_{1}^{\geq 0}\right)$ is another $\mathbf{H}$-invariant 
$t$-structure on $D^{b}(B)$ such that $\Phi\left(D^{[a, b]}\right) \subset{ }^{\Phi} D_{1}^{[a, b]}$. Then applying [29, Theorem 2.1.2] again we deduce that

$$
D_{1}^{[a, b]}=\left\{F \in D^{b}(A) \mid \Phi(F) \in{ }^{\Phi} D_{1}^{[a, b]}\right\}
$$

is a $t$-structure on $D^{b}(A)$ such that $D^{[a, b]} \subset D_{1}^{[a, b]}$. Hence, $D_{1}^{[a, b]}=D^{[a, b]}$ and we can rewrite $(3.1 .2)$ as

$$
{ }^{\Phi} D^{[a, b]}=\left\{F \in D^{b}(B) \mid \Phi \Phi^{\prime}(F) \in{ }^{\Phi} D_{1}^{[a, b]}\right\},
$$

which implies that ${ }^{\Phi} D_{1}^{[a, b]} \subset{ }^{\Phi} D^{[a, b]}$, so these $t$-structures are the same.

The result about slicings is proved analogously.

Let $\operatorname{Stab}^{\mathbf{H}}(A)$ denote the space of $\mathbf{H}$-invariant stability conditions on $A$. (It is known to be nonempty for $\operatorname{dim} A \leq 2$ and in some cases for $\operatorname{dim} A=3$, see [16].)

\section{DEFINITION 3.1.2}

For $g \in \mathbf{U}(\mathbb{Q})$ and a stability $\sigma=(P(\cdot), Z) \in \operatorname{Stab}^{\mathbf{H}}(A)$ we set

$$
g(\sigma)=\left({ }^{\Phi_{g}} P(\cdot), Z \circ \hat{\rho}(g)^{-1}\right),
$$

where $\hat{\rho}(g)$ is given by (2.1.17). By Proposition 3.1.1, this defines an action of $\widetilde{\mathbf{U}(\mathbb{Q})}$ on $\operatorname{Stab}^{\mathbf{H}}(A)$ such that the central element $1 \in \mathbb{Z} \subset \widetilde{\mathbf{U}(\mathbb{Q})}$ sends $(P(\cdot), Z)$ to $(P(\cdot)[1],-Z)$.

The restriction of the above action to the preimage of $\mathbf{U}(\mathbb{Z}) \subset \mathbf{U}(\mathbb{Q})$ is given by the standard action of the autoequivalence group of $D^{b}(A)$ on $\operatorname{Stab}(A)$ (see [6]).

PROPOSITION 3.1.3

For every $\widetilde{g} \in \widetilde{\mathbf{U}(\mathbb{Q})}$ the corresponding transformation of $\operatorname{Stab}^{\mathbf{H}}(A)$ is an isometry with respect to the generalized metric $d(\cdot, \cdot)$ introduced in [6, Proposition 8.1].

Proof

Note that the functor $\Phi_{g}$ sends Harder-Narasimhan constituents of $E$ with respect to $\sigma$ to those of $\Phi_{g}(E)$ with respect to $g(\sigma)$, and $Z\left(\hat{\rho}(g)^{-1}\left(\Phi_{g}(E)\right)\right)$ is a constant multiple (depending only on $g$ ) of $Z(E)$. Hence, $d\left(\sigma_{1}, \sigma_{2}\right) \leq$ $d\left(g\left(\sigma_{1}\right), g\left(\sigma_{2}\right)\right)$. Applying the same inequality to $g^{-1}$ and the pair $\left(g\left(\sigma_{1}\right), g\left(\sigma_{2}\right)\right)$ we deduce that it is in fact an equality.

PROPOSITION 3.1.4

Any LI object in $D^{b}(A)$ is semistable with respect to any full stability.

Proof

Let $E$ be an $(L, \alpha)$-invariant object in $D^{b}(A)$, where $(L, \alpha)$ is a Lagrangian pair (with $L \subset X_{A}$ ), and let $\sigma=(P(\cdot), Z$ ) be a full stability. We can assume that $Z$ takes values in $\mathbb{Q}+i \mathbb{Q} \subset \mathbb{C}$. Indeed, the set of such stabilities is dense in the connected component containing $\sigma$, and the semistability of $E$ is a closed condition 
on $\sigma$. Then for a dense set of real numbers $t$ (namely, those with $\tan (\pi t) \in \mathbb{Q}$ ) the abelian category $P((t, t+1])$ is Noetherian (see [1, Proposition 5.0.1]). Applying the construction of [29] we obtain for each such $t$ the associated constant family of $t$-structures over any base $S$, which is a certain $t$-structure on $D^{b}(A \times S)$, local over $S$ and such that its heart contains the pullback of $P((t, t+1])$ with respect to the projection $p_{1}: A \times S \rightarrow A$. Let us take as a base $S=L$, and consider the functor

$$
T_{(L, \alpha)}: D^{b}(A) \rightarrow D^{b}(A \times L)
$$

that associates with $F \in D^{b}(A)$ the natural family of objects $\mathcal{F}$ on $L \times A$ such that the restriction of $\mathcal{F}$ to $\{l\} \times A$ is $\alpha_{l} \otimes T_{l}(F)$ for $l \in L$. (Here, $\mathcal{F}$ is obtained from $F$ by taking the pullback with respect to the map $L \times A \rightarrow A:(l, x) \mapsto p_{A}(l)+x$ and then tensoring the result with a certain line bundle.) Since our stability is $\mathbf{H}-$ invariant (by [29, Corollary 3.5.2]), this functor is easily seen to be $t$-exact; that is, it sends $P((t, t+1])$ to the heart of the corresponding constant $t$-structure on $D^{b}(A \times L)$. By definition, an $(L, \alpha)$-invariance structure on $E$ is an isomorphism

$$
T_{(L, \alpha)}(E) \simeq p_{1}^{*} E .
$$

Since both sides are $t$-exact functors of $E$, we deduce that the truncations of $E$ with respect to our $t$-structure are still $(L, \alpha)$-invariant. Applying this for an appropriate set of phases $t$ we derive that all Harder-Narasimhan constituents of $E$ are $(L, \alpha)$-invariant. Let $E_{0}$ be one of them. Suppose that $E_{0}$ has cohomological range $[a, b]$ with respect to the standard $t$-structure. Then $H^{b} E_{0}$ and $H^{a} E_{0}$ are still $(L, \alpha)$-invariant, so we have a nonzero morphism $H^{b} E_{0} \rightarrow H^{a} E_{0}$ (see [31, Theorem 2.4.5]), which gives rise to a nonzero morphism

$$
E_{0}[b] \rightarrow H^{b} E_{0} \rightarrow H^{a} E_{0} \rightarrow E_{0}[a] .
$$

By the semistability of $E_{0}$ we should have $b \leq a$; that is, $E_{0}$ is cohomologically pure. Since $E_{0}$ is a direct sum of several copies of the generator $S_{L, \alpha}$, it follows that $S_{L, \alpha}$ is also semistable.

\section{2. $\mathbb{Z}$-covering of LG $(\mathbb{R})$}

Recall that the action of $\mathbf{U}(\mathbb{R})$ on $\mathbf{L G}(\mathbb{R})$ is transitive (see Proposition 1.4.3), so we have an identification

$$
\mathbf{L G}(\mathbb{R}) \simeq \mathbf{U}(\mathbb{R}) / \mathbf{P}^{-}(\mathbb{R}) .
$$

We have a natural lifting of $\mathbf{P}^{-}(\mathbb{R})$ to a closed subgroup of $U^{\Delta}$ (see Lemma 2.3.3). Therefore, the homogeneous space $U^{\Delta} / \mathbf{P}^{-}(\mathbb{R})$ is a $\mathbb{Z}$-covering of $\mathbf{L G}(\mathbb{R})$. Below we describe this $\mathbb{Z}$-covering explicitly using the homogeneous coordinates $(x: y)$ on $\mathbf{L G}(\mathbb{R})$ (see Section 1.4 ).

Namely, to every $L=(x: y) \in \mathbf{L G}(\mathbb{R})$ we associate a holomorphic function on $D_{A}$, defined up to rescaling by a positive constant,

$$
\delta(L)(\omega)=\operatorname{deg}(\hat{y}-\hat{x} \omega)=\operatorname{deg}(\omega x-y) \bmod \mathbb{R}_{>0},
$$


where $\omega \in D_{A}$. Note that if we change $(x: y)$ to $(x \alpha: y \alpha)$, then this function gets multiplied by $\operatorname{deg}(\alpha) \in \mathbb{R}_{>0}$. It is easy to see that for $g \in \mathbf{U}(\mathbb{R})$ one has

$$
\delta\left(g\left(0: \phi_{0}\right)\right)=\Delta\left(g^{-1}\right) \bmod \mathbb{R}_{>0},
$$

where $\phi_{0}: A \rightarrow \hat{A}$ is a polarization and $\Delta$ is the 1 -cocycle of $\mathbf{U}(\mathbb{R})$ with values in $\mathcal{O}^{*}\left(D_{A}\right)$ defined in Section 2.3. In particular, $\delta(L)(\omega) \neq 0$ for all $\omega \in D_{A}$.

LEMMA 3.2.1

For $L \in \mathbf{L G}(\mathbb{R})$ and $g \in \mathbf{U}(\mathbb{R})$ one has

$$
\delta(g L)(g(\omega))=\delta(L)(\omega) \cdot \Delta\left(g^{-1}\right)(g(\omega))=\delta(L)(\omega) \cdot \Delta(g)(\omega)^{-1}
$$

Proof

Pick $g^{\prime} \in \mathbf{U}(\mathbb{R})$ such that $L=g^{\prime}\left(0: \phi_{0}\right)$. Then use (3.2.2) and the cocycle condition for $\Delta$.

Note also that if we have a Lagrangian subvariety $L \subset A \times \hat{A}$, then viewing $L$ as a point in $\mathbf{L G}(\mathbb{Q})$ we have

$$
\delta(L)(\omega)=\operatorname{deg}\left(\omega p_{1}-p_{2}\right) \bmod \mathbb{R}_{>0},
$$

where $p_{1}: L \rightarrow A$ and $p_{2}: L \rightarrow \hat{A}$ are the projections, and we use the polynomial function $\operatorname{deg}: \operatorname{Hom}(L, \hat{A}) \otimes \mathbb{C} \rightarrow \mathbb{C}$.

\section{DEFINITION 3.2.2}

We define the $\mathbb{Z}$-covering $p: \widetilde{\mathbf{L G}(\mathbb{R})} \rightarrow \mathbf{L G}(\mathbb{R})$ by setting

$$
\widetilde{\mathbf{L G}(\mathbb{R})}=\left\{(L, f) \in \mathbf{L G}(\mathbb{R}) \times\left(\mathcal{O}\left(D_{A}\right) / i \mathbb{R}\right) \mid \delta(L)=\exp (2 \pi i f) \bmod \mathbb{R}_{>0}\right\} .
$$

We also set

$$
\widetilde{\mathbf{L G}(\mathbb{Q})}:=p^{-1}(\mathbf{L G}(\mathbb{Q})) \subset \widetilde{\mathbf{L G}(\mathbb{R})} .
$$

When we need to stress the dependence on $A$ we write $\widetilde{\mathbf{L} \mathbf{G}_{A}(\mathbb{R})}\left(\operatorname{resp} ., \widetilde{\mathbf{L} \mathbf{G}_{A}(\mathbb{Q})}\right)$. We have an action of $U^{\Delta}$ on $\widetilde{\mathbf{L G}(\mathbb{R})}$ given by

$$
\left(g, f_{g}\right) \cdot\left(L, f_{L}\right)=\left(g L, f_{L}\left(g^{-1}(\omega)\right)+f_{g}\left(g^{-1}(\omega)\right)\right) .
$$

The fact that this action is well defined follows from Lemma 3.2.1.

PROPOSITION 3.2.3

(i) There exists a unique bijection

$$
\overline{\mathrm{SH}}^{\mathrm{LI}}(A) / \mathbb{N}^{*} \rightarrow \widetilde{\mathbf{L G}(\mathbb{Q})}: F \mapsto \widetilde{L}_{F}
$$

that lifts the natural projection $F \mapsto L_{F}$ to $\mathbf{L G}(\mathbb{Q})$, sends $\mathcal{O}_{x}$ to $\left(\left(0: \phi_{0}\right), 0\right) \in$ $\widehat{\mathbf{L G}(\mathbb{Q})}$ (where $\phi_{0}: A \rightarrow \hat{A}$ is a polarization), and is $\widehat{\mathbf{U}(\mathbb{Q})}$-equivariant, where the action on $\widehat{\mathbf{L G}(\mathbb{Q})}$ is induced by the embedding $\iota: \widetilde{\mathbf{U}(\mathbb{Q})} \rightarrow U^{\Delta}$. 
(ii) Let $V_{\phi}$ be the semihomogeneous vector bundle associated with $\phi \in \operatorname{NS}(A) \otimes \mathbb{Q}$, so that $L_{V_{\phi}}=\Gamma(\phi)$ (see (2.1.4)). Then

$$
\widetilde{L}_{V_{\phi}}=\left(\Gamma(\phi),(2 \pi i)^{-1} \cdot \log (\operatorname{deg}(\omega-\phi)) \bmod i \mathbb{R}\right),
$$

where the branch of $\log (\operatorname{deg}(\cdot))$ is normalized by

$$
\operatorname{Im} \log (\operatorname{deg}(i H))=\operatorname{Arg}(\operatorname{deg}(i H))=-g \pi
$$

for ample $H$.

\section{Proof}

(i) First, let us compute the stabilizer subgroup $\mathrm{St} \subset \widetilde{\mathbf{U}(\mathbb{Q})}$ of the class of $\mathcal{O}_{x}$ in $\overline{\mathrm{SH}}^{\mathrm{LI}}(A) / \mathbb{N}^{*}$. By considering the action on the corresponding Lagrangian we see that $\mathrm{St}$ is a certain lifting of $\mathbf{P}^{-}(\mathbb{Q}) \subset \mathbf{U}(\mathbb{Q})$ to $\widehat{\mathbf{U}(\mathbb{Q})}$. From the explicit form of the functors $\Phi_{t}$ for $t \in \mathbf{T}(\mathbb{Q})$ (see Proposition 2.2.1(ii)) we see that these functors preserve $\mathcal{O}_{x}$ up to $\mathbf{H}$-equivalence and $\mathbb{N}^{*}$. Therefore, St is the lifting of $\mathbf{P}^{-}(\mathbb{Q})$ described in Corollary 2.2.2. By Lemma 2.3.3, $\iota(\mathrm{St})$ is exactly the stabilizer of the point $\left(\left(0: \phi_{0}\right), 0\right) \in \widetilde{\mathbf{L G}(\mathbb{Q})}$. Hence, there is a well-defined $\widetilde{\mathbf{U}(\mathbb{Q})}$-equivariant map (3.2.4). Since this is a map of $\mathbb{Z}$-torsors over $\mathbf{L G}(\mathbb{Q})$ (see Proposition 2.1.2), it is a bijection.

(ii) Assume first that $\phi$ is nondegenerate, that is, that $\phi \in \operatorname{NS}^{0}(A, \mathbb{Q})$. Consider the element $g_{\phi^{-1}}^{+} \in \mathbf{N}^{+}(\mathbb{Q})$ as in Proposition 2.2.1(i). Then

$$
g_{\phi^{-1}}^{+}\left(0: \phi_{0}\right)=\left(\phi^{-1} \phi_{0}: \phi_{0}\right)=(1: \phi)=\Gamma(\phi) .
$$

By Proposition 2.2.1(i), under the canonical lifting of $\mathbf{N}^{+}(\mathbb{Q})$ to $\widetilde{\mathbf{U}(\mathbb{Q})}$ the lifting of $g_{\phi^{-1}}^{+}$corresponds to the kernel $S\left(g_{\phi^{-1}}^{+}\right)[i(\phi)]$. (Note that $i\left(\phi^{-1}\right)=i(\phi)$.) On the other hand, its canonical lifting to $U^{\Delta}$ is

$$
\widetilde{g}=\left(g_{\phi^{-1}}^{+},-\log \left(\operatorname{deg}\left(1+\phi^{-1} \omega\right)\right) / 2 \pi i\right),
$$

where we use the branch of $\operatorname{Arg} \operatorname{deg}\left(1+\phi^{-1} \omega\right)$ that tends to 0 as $\omega \rightarrow 0$ (see Lemma 2.3.4). By the $\widetilde{\mathbf{U}(\mathbb{Q})}$-equivariance of the map (3.2.4), we obtain that the object $V_{\phi}[i(\phi)]$ is mapped under this map to

$$
\begin{aligned}
\widetilde{g} \cdot\left(\left(0: \phi_{0}\right), 0\right) & =\left(\Gamma(\phi), \log \left(\operatorname{deg}\left(1-\phi^{-1} \omega\right)\right) / 2 \pi i \bmod i \mathbb{R}\right) \\
& =\left(\Gamma(\phi),(2 \pi i)^{-1} \cdot \log (\operatorname{deg}(\omega-\phi)) \bmod i \mathbb{R}\right)
\end{aligned}
$$

with the same choice of the argument as above. Recall that if we choose the branch of $\operatorname{Arg} \operatorname{deg}(\omega-\phi)$ in such a way that $\operatorname{Arg} \operatorname{deg}(i n H-\phi)$ will be $\pi g$, then we will obtain (see Corollary 1.2.2)

$$
\operatorname{Arg} \operatorname{deg}(-\phi)=2 \pi i(-\phi)=2 \pi(g-i(\phi)) .
$$

Subtracting $2 \pi g$ we get the branch that gives the limit $-2 \pi i(\phi)$ as $\omega \rightarrow 0$, which is exactly what we get for the image of $V_{\phi}$. This proves the required statement in the case when $\phi \in \operatorname{NS}^{0}(A, \mathbb{Q})$. The general case follows by using the action of 
the subgroup $\mathbf{N}^{-}(\mathbb{Q}) \subset \widetilde{\mathbf{U}(\mathbb{Q})}$ (see Example 2.1.3). Indeed, this action changes both sides (3.2.5) by adding to $\phi$ an arbitrary element of $\mathrm{NS}(A) \otimes \mathbb{Q}$.

Recall that we can view $\mathrm{NS}(A) \otimes \mathbb{R}$ as an open subset of $\mathbf{L G}(\mathbb{R})$ via the map $\phi \mapsto(1: \phi)=\Gamma(\phi)$. Proposition 3.2.3(ii) implies that we have a commutative diagram

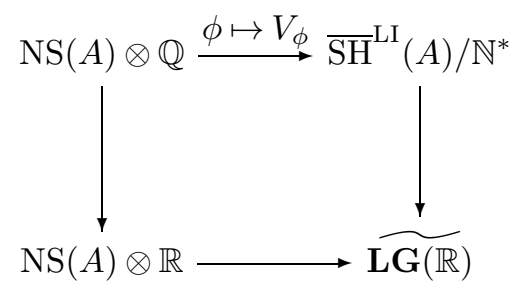

where the right vertical arrow is (3.2.4) and the bottom arrow is the continuous section of the projection $\widehat{\mathbf{L G}(\mathbb{R})} \rightarrow \mathbf{L G}(\mathbb{R})$ over $\mathrm{NS}(A) \otimes \mathbb{R}$ given by

$$
\mathrm{NS}(A) \otimes \mathbb{R} \rightarrow \widetilde{\mathbf{L G}(\mathbb{R})}: \phi \mapsto\left(\Gamma(\phi), f_{\phi}\right),
$$

where $f_{\phi} \in \mathcal{O}\left(D_{A}\right) \bmod i \mathbb{R}$ is the branch of $(2 \pi i)^{-1} \cdot \log (\operatorname{deg}(\omega-\phi)) \bmod i \mathbb{R}$ satisfying

$$
\lim _{n \rightarrow \infty} f_{\phi}(i n H)=-g / 2
$$

for any ample $H$.

\section{DEFINITION 3.2.4}

We define the double covering $p^{\text {spin }}: \mathbf{L G}^{\text {spin }}(A, \mathbb{R}) \rightarrow \mathbf{L G}_{A}(\mathbb{R})$ by setting $\mathbf{L G}^{\text {spin }}(A, \mathbb{R})=\widehat{\mathbf{L G}(\mathbb{R})} / 2 \mathbb{Z}$. Explicitly,

$$
\mathbf{L G}^{\text {spin }}(A, \mathbb{R})=\left\{(L, \varphi) \in \mathbf{L G}(\mathbb{R}) \times\left(\mathcal{O}\left(D_{A}\right) / \mathbb{R}_{>0}\right) \mid \delta(L)=\varphi^{2} \bmod \mathbb{R}_{>0}\right\} .
$$

We also set $\mathbf{L G}^{\text {spin }}(A, \mathbb{Q})=\left(p^{\text {spin }}\right)^{-1}(\mathbf{L G}(\mathbb{Q}))$.

The isomorphism $\operatorname{Spin}(\mathbb{R}) \simeq U^{\Delta} / 2 \mathbb{Z}$ induces a transitive action of $\operatorname{Spin}(\mathbb{R})$ on $\mathbf{L} \mathbf{G}^{\text {spin }}(A, \mathbb{R})$ (and of $\mathbf{U}(\mathbb{Q})^{\text {spin }}$ on $\mathbf{L} \mathbf{G}^{\text {spin }}(A, \mathbb{Q})$ ).

We also have a natural $\widetilde{\mathbf{U}(\mathbb{Q})}$-equivariant map (see $(2.4 .8)$ )

$$
\overline{\mathrm{SH}}^{\mathrm{LI}}(A) / \mathbb{N}^{*} \rightarrow \mathcal{N}(A) \otimes \mathbb{Q} / \mathbb{Q}_{>0}: F \mapsto[F] \bmod \mathbb{Q}_{>0},
$$

which we can view as a map from $\widehat{\mathbf{L G}(\mathbb{Q})}$ using the bijection (3.2.4). The equivariance of this map with respect to the $\mathbb{Z}$-action implies that it factors through $\mathbf{L G}^{\text {spin }}(A, \mathbb{Q})$. Furthermore, we claim that it extends to a continuous $\operatorname{Spin}(\mathbb{R})$ equivariant map

$$
\mathbf{L G}^{\text {spin }}(A, \mathbb{R}) \rightarrow \mathcal{N}(A) \otimes \mathbb{R} / \mathbb{R}_{>0}
$$


such that we have a commutative diagram

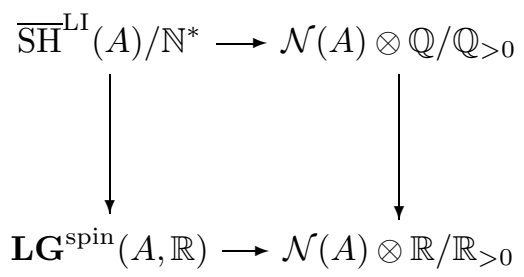

Indeed, we can define $(3.2 .7)$ by sending $\widetilde{g}\left(\left(0: \phi_{0}\right), 1\right)$ to $\widetilde{g}\left[\mathcal{O}_{x}\right] \bmod \mathbb{R}_{>0}$ for $\widetilde{g} \in$ $\operatorname{Spin}(\mathbb{R})$. To check that this map is well defined we observe that $\mathbb{Q}$-points $\mathbf{P}^{-}(\mathbb{Q})$ are dense (with respect to the classical topology) in the stabilizer $\mathbf{P}^{-}(\mathbb{R})$ of the point $\left(\left(0: \phi_{0}\right), 1\right) \in \mathbf{L} \mathbf{G}^{\text {spin }}(A, \mathbb{R})$. Since $\mathbf{P}^{-}(\mathbb{Q}) \subset \mathbf{U}(\mathbb{Q})^{\text {spin }}$ leaves the class $\left[\mathcal{O}_{x}\right] \in S \mathcal{N}(A) \otimes \mathbb{R}$ invariant, this proves our claim.

\section{LEMMA 3.2.5}

The section (3.2.6) induces a section

$$
\mathrm{NS}(A) \otimes \mathbb{R} \rightarrow \mathbf{L G}^{\text {spin }}(A, \mathbb{R}),
$$

which sends $\phi \in \mathrm{NS}(A) \otimes \mathbb{R}$ to $\left(\Gamma(\phi), \chi(\phi-\omega) \bmod \mathbb{R}_{>0}\right)$.

Proof

Since $\chi(\phi-\omega)^{2}=\operatorname{deg}(\phi-\omega)=\operatorname{deg}(\omega-\phi)$, this follows from the fact that the argument of $\chi(\phi-i n H)$ tends to $-g \pi / 2 \bmod 2 \pi \mathbb{Z}$ as $n \rightarrow \infty$.

\section{EXAMPLE 3.2.6}

In the case when $A=E^{n}$, where $E$ is an elliptic curve without complex multiplication, we can identify $\operatorname{End}(A)$ with the algebra of $n \times n$ matrices over $\mathbb{Z}$, and $\operatorname{NS}(A)$ with symmetric matrices. Note that for $M \in \operatorname{End}(A)$ we have $\operatorname{deg}(M)=\operatorname{det}(M)^{2}$ and for $\phi \in \mathrm{NS}(A)$ we have $\chi(\phi)=\operatorname{det}(\phi)$. In a coordinatefree notation, if $A=E \otimes \Lambda$, where $\Lambda$ is a free $\mathbb{Z}$-module of rank $n$, then elements of $\operatorname{NS}(A)$ can be viewed as $\mathbb{Z}$-valued symmetric bilinear forms on $\Lambda$, and the function $\chi$ is given by the discriminant. The group $\mathbf{U}$ in this case is the symplectic group $\mathrm{Sp}_{2 n}$ and the variety $\mathbf{L G}_{A}$ is the Lagrangian Grassmannian associated with the $2 n$-dimensional symplectic vector space. Also, $D_{A}$ is the Siegel upper half-plane $\mathfrak{H}_{n}$, and the covering $U^{\Delta} \rightarrow \mathrm{Sp}_{2 n}$ corresponds to a choice of argument

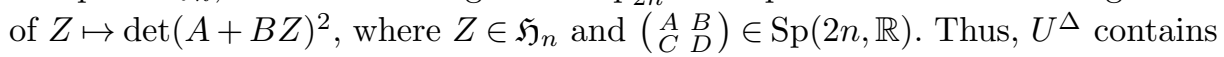
the universal covering $\widetilde{\mathrm{Sp}}(2 n, \mathbb{R})$ of $\operatorname{Sp}(2 n, \mathbb{R})$ as a subgroup of index 2 (cf. [23, Example 4.15]). Now let us consider our lifting of $\mathbf{P}^{-}(\mathbb{R})$ to $U^{\Delta}$. It is easy to check that the restriction of the projection to $U^{\Delta} \rightarrow U^{\Delta} / \widetilde{\mathrm{Sp}}(2 n, \mathbb{R}) \simeq\{ \pm 1\}$ to $\mathrm{GL}(n, \mathbb{R}) \subset \mathbf{P}^{-}(\mathbb{R})$ can be identified with the homomorphism $A \mapsto \operatorname{sign} \operatorname{det}(A)$. It follows that $U^{\Delta}=\widetilde{\operatorname{Sp}}(2 n, \mathbb{R}) \cdot \mathbf{P}^{-}(\mathbb{R})$, and $\mathbf{P}^{-}(\mathbb{R}) \cap \widetilde{\mathrm{Sp}}(2 n, \mathbb{R})$ is the semidirect product of $\mathbf{N}^{-}(\mathbb{R})$ and of $\mathrm{GL}^{+}(n, \mathbb{R})$ (matrices with positive determinant). Hence, we can identify $\widetilde{\mathbf{L} \mathbf{G}_{A}(\mathbb{R})}$ with the quotient of $\widetilde{\operatorname{Sp}}(2 n, \mathbb{R})$ by a connected subgroup, 
so $\widetilde{\mathbf{L} \mathbf{G}_{A}(\mathbb{R})}$ is simply connected. It follows that in this case $\widetilde{\mathbf{L} \mathbf{G}_{A}(\mathbb{R})}$ is the universal covering of the Lagrangian Grassmannian $\mathbf{L G}_{A}(\mathbb{R})$.

\subsection{Phase function}

Since $\Delta: \mathbf{U}(\mathbb{R}) \rightarrow \mathcal{O}^{*}\left(D_{A}\right)$ is a 1-cocycle (see Lemma 2.3.1), it defines a natural action of the group $\mathbf{U}(\mathbb{R})$ (by holomorphic automorphisms) on the trivial $\mathbb{C}^{*}$ bundle over $D_{A}$. We have constructed the central extension $U^{\Delta} \rightarrow \mathbf{U}(\mathbb{R})$ by $\mathbb{Z}$ in such a way that $\Delta$ lifts to a 1-cocycle of $U^{\Delta}$ with coefficients in $\mathcal{O}\left(D_{A}\right)$. In other words, we obtain the action of $U^{\Delta}$ on $D_{A} \times \mathbb{C}$ (respecting the structure of a $\mathbb{C}$-space), which we view as a universal covering of $D_{A} \times \mathbb{C}^{*}$. (In Section 3.4 we relate this covering to Bridgeland's stability space in the case $\operatorname{dim} A=2$.) Explicitly, this action is given by

$$
(g, f) \cdot(\omega, z)=(g(\omega), z-f(\omega)),
$$

where $(g, f) \in U^{\Delta}$ and $(\omega, z) \in D_{A} \times \mathbb{C}$.

On the other hand, we have a transitive action of $U^{\Delta}$ on the $\mathbb{Z}$-covering $\widehat{\mathbf{L G}(\mathbb{R})}$ of $\mathbf{L G}(\mathbb{R})$. By definition of this $\mathbb{Z}$-covering, we have a continuous function

$$
\mathbf{f}_{0}: D_{A} \times \widetilde{\mathbf{L G}(\mathbb{R})} \rightarrow \mathbb{R}:\left(\omega,\left(L, f_{L}\right)\right) \mapsto \operatorname{Re} f_{L}(\omega) .
$$

We can extend it to a continuous function on $\left(D_{A} \times \mathbb{C}\right) \times \widetilde{\mathbf{L G}(\mathbb{R})}$ setting

$$
\mathbf{f}((\omega, z), \widetilde{L})=\operatorname{Re}(z)+\mathbf{f}_{0}(\omega, \widetilde{L})
$$

where $\widetilde{L} \in \widetilde{\mathbf{L G}(\mathbb{R})}$.

LEMMA 3.3.1

The function $\mathbf{f}$ is $U^{\Delta}$-invariant; that is, for $\widetilde{g} \in U^{\Delta}$ and $(\sigma, \widetilde{L}) \in\left(D_{A} \times \mathbb{C}\right) \times$ $\widetilde{\mathbf{L G}(\mathbb{R})}$ one has

$$
\mathbf{f}(\widetilde{g}(\sigma), \widetilde{g}(\widetilde{L}))=\mathbf{f}(\sigma, \widetilde{L})
$$

The proof is straightforward.

Now using the map $F \mapsto \widetilde{L}_{F}$ of Proposition 3.2.3, we define the phase function

$$
\left(D_{A} \times \mathbb{C}\right) \times \overline{\mathrm{SH}}^{\mathrm{LI}}(A) / \mathbb{N}^{*} \rightarrow \mathbb{R}:(\sigma, \widetilde{L}) \mapsto \phi_{\sigma}(F):=\mathbf{f}\left(\sigma, \widetilde{L}_{F}\right),
$$

where $\sigma \in D_{A} \times \mathbb{C}$. Note that we have

$$
\phi_{(\omega, z)}(F)=\phi_{(\omega, 0)}(F)+\operatorname{Re}(z) .
$$

In Section 3.4 we show that in the surface case the function $\phi_{\sigma}$ gives the phases of LI objects with respect to the Bridgeland stability condition on $D^{b}(A)$ associated with $\sigma \in D_{A} \times \mathbb{C}$. In the following theorem we check some of the properties of $\phi_{\sigma}$ that conform to the conjecture that the corresponding stability condition exists in the higher-dimensional case as well. 


\section{THEOREM 3.3.2}

The phase function $\phi_{\sigma}(F)$ satisfies the following properties.

(i) This function is $\widetilde{\mathbf{U}(\mathbb{Q})}$-invariant, that is;

$$
\phi_{\widetilde{g}(\sigma)}(\widetilde{g}(F))=\phi_{\sigma}(F),
$$

where the action of $\widetilde{\mathbf{U}(\mathbb{Q})}$ on $D_{A} \times \mathbb{C}$ is induced by (3.3.1) via the homomorphism $\iota: \widetilde{\mathbf{U}(\mathbb{Q})} \rightarrow U^{\Delta}$. In particular, for $n \in \mathbb{Z}$

$$
\phi_{\sigma}(F[n])=\phi_{\sigma}(F)+n \text {. }
$$

(ii) For $\sigma=(\omega, z)$ and $F \in \overline{\mathrm{SH}}^{\mathrm{LI}}(A)$ one has

$$
\exp (\pi i z) \cdot \chi(\ell(\omega),[F]) \in \mathbb{R}_{>0} \cdot \exp \left(\pi i \phi_{\sigma}(F)\right),
$$

where $[F] \in \mathcal{N}(A) \otimes \mathbb{R}$ is the numerical class of $F$.

(iii) For a semihomogeneous vector bundle $V_{\phi}$ associated with $\phi \in \mathrm{NS}(A) \otimes \mathbb{Q}$ one has

$$
\phi_{(\omega, z)}\left(V_{\phi}\right)=\operatorname{Re}(z)+\frac{1}{2 \pi} \operatorname{Arg}(\operatorname{deg}(\omega-\phi)),
$$

where the branch of $\operatorname{Arg}(\operatorname{deg}(\cdot))$ is normalized by $\operatorname{Arg}(\operatorname{deg}(i H))=-g \pi$ for ample $H$.

(iv) For a pair of LI objects $F_{1}$ and $F_{2}$ such that the corresponding Lagrangians $L_{F_{1}}$ and $L_{F_{2}}$ in $A \times \hat{A}$ are transversal, one has

$$
\phi_{\sigma}\left(F_{1}\right) \leq \phi_{\sigma}\left(F_{2}\right)+i\left(F_{1}, F_{2}\right),
$$

where $i\left(F_{1}, F_{2}\right)$ is the index of the pair $\left(F_{1}, F_{2}\right)$, that is, the number such that $\operatorname{Ext}^{i}\left(F_{1}, F_{2}\right)=0$ for $i \neq i\left(F_{1}, F_{2}\right)$. (It exists by [31, Corollary 3.2.12].)

Proof

(i) The invariance follows from Lemma 3.3.1. The second assertion follows from this:

$$
\phi_{(\omega, z)}(F)=\phi_{(1, n) \cdot(\omega, z)}(F[n])=\phi_{(\omega, z-n)}(F[n])=\phi_{(\omega, z)}(F[n])-n,
$$

where in the last equality we used (3.3.3).

(ii) By part (i), the right-hand side of (3.3.4) is invariant under the diagonal action of $\widetilde{\mathbf{U}(\mathbb{Q})}$ on $(\sigma, F)$. We claim that the same is true for the left-hand side (modulo $\left.\mathbb{R}_{>0}\right)$. Indeed, by Corollary 2.5.6, for $\widetilde{g}=\left(g, f_{g}\right) \in U^{\Delta}$ we have

$$
\chi(\ell(\omega),[F])=\exp \left(-\pi i f_{g}(\omega)\right) \cdot \chi(\ell(g(\omega)),[\widetilde{g}(F)]) \bmod \mathbb{R}_{>0} .
$$

(Recall that the map $F \mapsto[F] \bmod \mathbb{N}^{*}$ is compatible with the projection $U^{\Delta} \rightarrow$ $\operatorname{Spin}(\mathbb{R})$ sending $\left(g, f_{g}\right) \in U^{\Delta}$ to $\left(g, \exp \left(-\pi i f_{g}\right)\right)$ (see $\left.(2.3 .3)\right)$.) This immediately implies that the left-hand side of (3.3.4) is invariant modulo $\mathbb{R}_{>0}$ with respect to the diagonal action of $\widetilde{\mathbf{U}(\mathbb{Q})}$ on the pair $(\sigma, F) \in\left(D_{A} \times \mathbb{C}\right) \times \overline{\mathrm{SH}}^{\mathrm{LI}}(A) / \mathbb{N}^{*}$.

Thus, it is enough to check the equality for $F=\mathcal{O}_{x}$. We have $\chi\left(\ell(\omega),\left[\mathcal{O}_{x}\right]\right)=1$ for all $\omega$. On the other hand, by the definition of the map of Proposition 3.2.3, $\mathbf{f}_{0}\left(\omega, \widetilde{L}_{\mathcal{O}_{x}}\right)=0$, so $\phi_{(\omega, z)}\left(\mathcal{O}_{x}\right)=\operatorname{Re}(z)$. 
(iii) This follows from Proposition 3.2.3(ii).

(iv) By the $\widehat{\mathbf{U}(\mathbb{Q})}$-invariance of both parts with respect to the diagonal action on the pair $\left(F_{1}, F_{2}\right)$, it is enough to consider the case when $F_{2}=\mathcal{O}_{x}$. Note that in this case the transversality assumption implies that $L_{F_{1}}=\Gamma(\phi)$ for $\phi \in \mathrm{NS}(A)_{\mathbb{Q}}$, so $F_{1}=V_{\phi}[n]$ for some $n \in \mathbb{Z}$, where $V_{\phi}$ is the simple semihomogeneous bundle associated with $\phi$. Since $\phi_{(\omega, z)}\left(\mathcal{O}_{x}\right)=\operatorname{Re}(z)$, by part (iii), the required inequality is equivalent to

$$
\operatorname{Arg}(\operatorname{deg}(\omega-\phi)) \leq 0,
$$

where $\operatorname{Arg}(\operatorname{deg}(\cdot))$ is normalized by $\operatorname{Arg}(\operatorname{deg}(i H))=-g \pi$. But this follows immediately from Lemma 1.2.3(ii).

\section{REMARK 3.3.3}

By Lemma 1.2.3(i), for $F_{1}=\mathcal{O}, F_{2}=V_{\phi}$, and $\sigma=(i H, 0)$, where $H$ is an ample class, the inequality (3.3.5) can be replaced by a stronger one:

$$
\phi_{i H, 0}(\mathcal{O})<\phi_{i H, 0}\left(V_{\phi}\right)+\frac{i(\phi)}{2} .
$$

However, this inequality is not invariant with respect to the group action considered above, so it cannot be extended to the case of arbitrary $\sigma \in D_{A} \times \mathbb{C}$.

The following property is also motivated by the picture with the stability conditions for $\operatorname{dim} A=2$ (see Section 3.4 below).

PROPOSITION 3.3.4

The fibers of the map

$$
\mathcal{Z}: D_{A} \times \mathbb{C} \rightarrow \operatorname{Hom}(\mathcal{N}(A), \mathbb{C}):(\omega, z) \mapsto \exp (\pi i z) \chi(\ell(\omega), \cdot)
$$

are exactly the orbits of the action of $2 \mathbb{Z} \subset \mathbb{C}$ by translations on the second factor.

Proof

Suppose that

$$
\exp (\pi i z) \chi(\ell(\omega),[F])=\exp \left(\pi i z^{\prime}\right) \chi\left(\ell\left(\omega^{\prime}\right),[F]\right)
$$

for all $F$. Since $\chi\left(\ell(\cdot),\left[\mathcal{O}_{x}\right]\right)=1$, this implies that $\exp (\pi i z)=\exp \left(\pi i z^{\prime}\right)$. Using the action of $2 \mathbb{Z}$ we can assume that $z=z^{\prime}$. Now the fact that $\omega=\omega^{\prime}$ follows from Corollary 1.1.2.

\section{EXAMPLE 3.3.5}

Recall that the standard stability condition on an elliptic curve has $Z(F)=$ $-\operatorname{deg}(F)+i \operatorname{rk}(F)$ and semistable objects that are shifts of semistable bundles and torsion sheaves. The corresponding phase function $\phi^{s t}$ satisfies

$$
\phi^{s t}(F)=\phi_{(i, 0)}(F)+1
$$


for any semistable $F$. Indeed, this follows from the formulas

$$
\phi^{s t}\left(\mathcal{O}_{x}\right)=1, \phi^{s t}\left(V_{d / r}\right)=\frac{\operatorname{Arg}(i-d / r)}{\pi},
$$

where $V_{d / r}$ is the simple bundle of degree $d$ and rank $r$ and we normalize the argument in the upper half-plane by $\operatorname{Arg}(i)=1 / 2$.

\subsection{Stability conditions on abelian surfaces}

In this section, assuming that $\operatorname{dim} A=2$ we will identify the action of $\iota(\widetilde{\mathbf{U}(\mathbb{Q})}) \subset$ $U^{\Delta}$ on $D_{A} \times \mathbb{C}$ with the natural action of $\widetilde{\mathbf{U}(\mathbb{Q})}$ on the component $\mathrm{Stab}^{\dagger}$ of Bridgeland's stability space $\operatorname{Stab}(A)$ of $D^{b}(A)$ described in [7, Section 15].

Recall that the stability $\operatorname{space} \operatorname{Stab}(A)$ carries a natural continuous action of the group $\widetilde{G L}^{+}(2, \mathbb{R})$, the universal cover of $\mathrm{GL}^{+}(2, \mathbb{R})$, which can be described as the set of pairs $(T, f)$, where $T \in \mathrm{GL}^{+}(2, \mathbb{R})$ and $f: \mathbb{R} \rightarrow R$ is an increasing map with $f(t+1)=f(t)+1$ such that the map induced by $T$ on $\mathbb{R}^{2} \backslash\{0\} / \mathbb{R}_{>0} \simeq \mathbb{R} / 2 \mathbb{Z}$ coincides with $f \bmod 2 \mathbb{Z}$. We use the left action of $\widetilde{\mathrm{GL}}^{+}(2, \mathbb{R})$ on $\operatorname{Stab}(A)$ : a pair $(T, f)$ maps a stability condition $(Z, \mathcal{P})$ to the stability $\left(T \circ Z, \mathcal{P}^{\prime}\right)$, where $\mathcal{P}^{\prime}(t)=$ $\mathcal{P}\left(f^{-1}(t)\right)$. Note that $n \mapsto\left((-1)^{n}, t \mapsto t+n\right)$ gives an embedding $\mathbb{Z} \rightarrow \widetilde{\mathrm{GL}}^{+}(2, \mathbb{R})$ such that $2 \mathbb{Z}$ is the kernel of the projection to $\mathrm{GL}^{+}(2, \mathbb{R})$.

Recall that for each $\omega=i \alpha+\beta \in D_{A}$ Bridgeland defined a stability condition on $D^{b}(A)$ with the central charge

$$
Z_{\omega}(F)=-\chi(\ell(\omega),[F])
$$

and with each $\mathcal{O}_{x}$ stable of phase 1 . This defines a submanifold $V(A) \subset \operatorname{Stab}(A)$, isomorphic to $D_{A}$, which is a section of the action of $\widetilde{\mathrm{GL}}^{+}(2, \mathbb{R})$ on a connected component $\operatorname{Stab}^{\dagger}(A) \subset \operatorname{Stab}(A)$, so that we have an isomorphism (see [7, Sections 11 and 15])

$$
V(A) \times \widetilde{\mathrm{GL}}^{+}(2, \mathbb{R}) \simeq \operatorname{Stab}^{\dagger}(A) .
$$

Conjecturally, $\operatorname{Stab}^{\dagger}(A)=\operatorname{Stab}(A)$. Below we show that $\operatorname{Stab}^{\dagger}(A)$ contains all full stabilities (see Proposition 3.4.4).

We have a natural embedding $\mathbb{C}^{*}=\mathrm{GL}(1, \mathbb{C}) \rightarrow \mathrm{GL}^{+}(2, \mathbb{R})$ and the corresponding homomorphism of universal coverings $\mathbb{C} \hookrightarrow \widetilde{\mathrm{GL}}^{+}(2, \mathbb{R})$ (where we use the map $\left.\mathbb{C} \rightarrow \mathbb{C}^{*}: z \mapsto \exp (\pi i z)\right)$. Hence, from the isomorphism (3.4.1) we obtain an embedding

$$
D_{A} \times \mathbb{C} \simeq V(A) \times \mathbb{C} \hookrightarrow \operatorname{Stab}^{\dagger}(A) .
$$

Note that the central charge corresponding to a point $(\omega, z) \in D_{A} \times \mathbb{C}$ is

$$
Z_{(\omega, z)}(F)=-\exp (\pi i z) \chi(\ell(\omega),[F]),
$$

and the phase of $\mathcal{O}_{x}$ with respect to this stability is

$$
\phi_{(\omega, z)}^{B r}\left(\mathcal{O}_{x}\right)=1+\operatorname{Re}(z) .
$$


Recall that the nonempty fibers of the projection

$$
\mathcal{Z}: \operatorname{Stab}^{\dagger}(A) \rightarrow \operatorname{Hom}(\mathcal{N}(A), \mathbb{C})
$$

are exactly the orbits of the action of $2 \mathbb{Z} \subset \mathbb{C} \subset \widetilde{\mathrm{GL}}^{+}(2, \mathbb{R})$ (see $[7$, Theorem 15.2]). Hence, we have

$$
V(A) \times \mathbb{C}=\mathcal{Z}^{-1}(\mathcal{Z}(V(A) \times \mathbb{C})) .
$$

The image $\mathcal{Z}(V(A) \times \mathbb{C})$ coincides with $\mathbb{C}^{*} \cdot \ell\left(D_{A}\right) \subset \mathcal{N}(A) \otimes \mathbb{C}$, where we identify $\mathcal{N}(A) \otimes \mathbb{C}$ with $\operatorname{Hom}(\mathcal{N}(A), \mathbb{C})$ using $\chi(\cdot, \cdot)$.

Recall that we have an action of the group $\widetilde{\mathbf{U}(\mathbb{Q})}$ on $\operatorname{Stab}^{\mathbf{H}}(A)$ defined using functors $\Phi_{g}$ (see Definition 3.1.2). Also, note that, by [29, Corollary 3.5.2], we have an inclusion $\operatorname{Stab}^{\dagger}(A) \subset \operatorname{Stab}^{\mathbf{H}}(A)$ since all stabilities in $\operatorname{Stab}^{\dagger}(A)$ are full.

\section{PROPOSITION 3.4.1}

The subset $V(A) \times \mathbb{C} \subset \operatorname{Stab}^{\mathbf{H}}(A)$ is invariant with respect to the action of $\widetilde{\mathbf{U}(\mathbb{Q})}$, and the induced action of $\widetilde{\mathbf{U}(\mathbb{Q})}$ on $V(A) \times \mathbb{C} \simeq D_{A} \times \mathbb{C}$ is exactly (3.3.1).

Proof

First, let us look at the action on central charges. Applying Corollary 2.5.6 to the element $\widetilde{g}=(g, \exp (-\pi i f)) \in \operatorname{Spin}(\mathbb{R})$ coming from an element $(g, f)=\iota\left(g^{\prime}\right) \in U^{\Delta}$ where $g^{\prime} \in \widehat{\mathbf{U}(\mathbb{Q})}$, we get (see $(3.3 .1)$ )

$$
Z_{(\omega, z)}\left(\hat{\rho}\left(g^{\prime}\right)^{-1} F\right)=Z_{(\omega, z)}\left(\hat{\rho}(\widetilde{g})^{-1}[F]\right)=Z_{\iota\left(g^{\prime}\right) \cdot(\omega, z)}(F) .
$$

In particular, the transformed central charge is still in $\mathbb{C}^{*} \cdot \ell\left(D_{A}\right)$. Recall that the connected component $\operatorname{Stab}^{\dagger}(A)$ is characterized by the condition that the central charge is in the $\mathrm{GL}^{+}(2, \mathbb{R})$-orbit of $\ell\left(D_{A}\right)$ and the $\mathcal{O}_{x}$ 's are stable of the same phase for all $x \in A$. Furthermore, by [7, Lemma 12.2], it is enough to require all $\mathcal{O}_{x}$ to be semistable of the same phase (due to the absence of spherical objects; see [7, Lemma 15.1]). In our case the condition on the central charge is satisfied by the above computation, and the semistability of $\mathcal{O}_{x}$ follows from Proposition 3.1.4, so we get the inclusion $g^{\prime}(V(A) \times \mathbb{C}) \subset$ Stab $^{\dagger}$. Taking into account (3.4.4) we derive the required inclusion

$$
g^{\prime}(V(A) \times \mathbb{C}) \subset V(A) \times \mathbb{C} \subset \mathrm{Stab}^{\dagger} .
$$

Furthermore, we obtain that the action of $g^{\prime} \in \widetilde{\mathbf{U}(\mathbb{Q})}$ on $D_{A} \times \mathbb{C}$ differs from the action (3.3.1) by the translation by an element in $2 \mathbb{Z} \subset \mathbb{C}$. Thus, the difference between the two actions is given by a homomorphism $\widehat{\mathbf{U}(\mathbb{Q})} \rightarrow 2 \mathbb{Z}$. Note that the element $1 \in \mathbb{Z} \subset \widehat{\mathbf{U}(\mathbb{Q})}$ acts on a stability in $\operatorname{Stab}(A)$ by changing the central charge $Z$ to $-Z$ and adding -1 to all the phases. Since this matches with its action on $D_{A} \times \mathbb{C}$ given by (3.3.1), the above homomorphism factors through a homomorphism $\mathbf{U}(\mathbb{Q}) \rightarrow 2 \mathbb{Z}$. Next, we observe that the action of $\mathbf{P}^{-}(\mathbb{Q}) \subset \widetilde{\mathbf{U}(\mathbb{Q})}$ preserves the phase of $\mathcal{O}_{x}$ (see the proof of Proposition 3.2.3(i)). On the other hand, $\iota\left(\mathbf{P}^{-}(\mathbb{Q})\right) \subset U^{\Delta}$ consists of elements $(g, f)$ with $\operatorname{Re}(f)=0$ 
(see Lemma 2.3.3), so taking into account the formula (3.4.3) we deduce that the homomorphism $\mathbf{U}(\mathbb{Q}) \rightarrow 2 \mathbb{Z}$ is trivial on $\mathbf{P}^{-}(\mathbb{Q})$. It remains to apply Lemma 1.3.5.

\section{COROLLARY 3.4.2}

There is a transitive continuous action of $U^{\Delta} \times \widetilde{\mathrm{GL}}_{2}^{+}(\mathbb{R})$ on $\operatorname{Stab}^{\dagger}(A)$, extending the action of $\widetilde{\mathrm{U}(\mathbb{Z})}$ (coming from autoequivalences of $D^{b}(A)$ ) and the standard action of $\widetilde{\mathrm{GL}}_{2}^{+}(\mathbb{R})$.

Proof

This follows from the identification (3.4.1) and from the transitivity of the action of $U^{\Delta}$ on $D_{A} \times \mathbb{C}$. Note that our action of $\widetilde{\mathbf{U}(\mathbb{Q})}$ on $\operatorname{Stab}^{\mathbf{H}}(A)$ extends the standard action of $\widetilde{\mathbf{U}(\mathbb{Z})}$ by autoequivalences of $D^{b}(A)$.

\section{THEOREM 3.4.3}

For any $\sigma=(\omega, z) \in D_{A} \times \mathbb{C}$ and any LI object $F \in D^{b}(A)$, let $\phi_{\sigma}^{B r}(F)$ be the phase of $F$ with respect to the corresponding Bridgeland stability condition. Then

$$
\phi_{\sigma}^{B r}(F)=\phi_{\sigma}(F)+1,
$$

where the function $\phi_{\sigma}$ is given by (3.3.2).

\section{Proof}

The assertion is true for $F=\mathcal{O}_{x}$. Also Theorem 3.3.2(i) together with Proposition 3.4.1 implies that both sides are invariant with respect to the action of $\widetilde{\mathbf{U}(\mathbb{Q})}$ on the pair $(\sigma, F)$. It remains to use transitivity of the action of $\widehat{\mathbf{U}(\mathbb{Q})}$ on $\overline{\mathrm{SH}}^{\mathrm{LI}}(A) / \mathbb{N}^{*}$.

We finish our consideration of abelian surfaces by observing that Proposition 3.1.4 implies the following description of the component $\operatorname{Stab}^{\dagger}(A)$.

\section{PROPOSITION 3.4.4}

For an abelian surface $A$ the component $\operatorname{Stab}^{\dagger}(A)$ of the stability space consists of all full stabilities on $D^{b}(A)$.

\section{Proof}

Let $\sigma$ be a full stability on $D^{b}(A)$. By Proposition 3.1.4, any skyscraper sheaf $\mathcal{O}_{x}$ is $\sigma$-semistable. Using [7, Lemmas 12.2 and 15.1] we deduce that $\mathcal{O}_{x}$ is in fact $\sigma$-stable. Now the proof of [7, Proposition 3.1.4] shows that any full stability belongs to $\operatorname{Stab}^{\dagger}(A)$. (One has to run this proof with $U(X)$ being the set of all full stabilities, which is an open subset of $\operatorname{Stab}(A)$.) 


\subsection{Mirror symmetry and phases}

In the case when $A=E^{n}$, where $E$ is an elliptic curve without complex multiplication, we can interpret the phase function of Section 3.3 in terms of the Fukaya category of the mirror dual abelian variety.

Let $E=\mathbb{C} /(\mathbb{Z}+\tau \mathbb{Z})$ be an elliptic curve over $\mathbb{C}$, and let $\Lambda$ be a free $\mathbb{Z}$-module of rank $n$. We set

$$
A=\Lambda \otimes \mathbb{C} /(\Lambda \otimes(\mathbb{Z}+\tau \mathbb{Z})) \simeq E^{n},
$$

so that we have a natural isomorphism

$$
\Gamma_{A}:=H_{1}(A, \mathbb{Z}) \simeq \Lambda \oplus \Lambda,
$$

where the second summand corresponds to $\Lambda \otimes \tau$. The natural polarization of $E$ given by the Hermitian form $H_{\tau}\left(z_{1}, z_{2}\right)=z_{1} \overline{z_{2}} /(\operatorname{Im} \tau)$ induces an isomorphism

$$
\hat{A} \simeq \Lambda^{*} \otimes \mathbb{C} /\left(\Lambda^{*} \otimes(\mathbb{Z}+\tau \mathbb{Z}),\right.
$$

where $\Lambda^{*}=\operatorname{Hom}_{\mathbb{Z}}(\Lambda, \mathbb{Z})$.

Assuming that $E$ has no complex multiplication we obtain identifications $\operatorname{End}(A) \simeq \operatorname{End}_{\mathbb{Z}}(\Lambda), \operatorname{Hom}(A, \hat{A}) \simeq \operatorname{Hom}_{\mathbb{Z}}\left(\Lambda, \Lambda^{*}\right)$, and $\operatorname{NS}(A) \simeq \operatorname{Hom}_{\mathbb{Z}}\left(\Lambda, \Lambda^{*}\right)^{+}$. (The latter group consists of symmetric homomorphisms.) Thus, for a field $F \supset \mathbb{Q}$ we can identify $\mathrm{NS}(A) \otimes F$ with the space of symmetric bilinear forms on $\Lambda \otimes F$. The ample cone in $\mathrm{NS}(A) \otimes \mathbb{R}$ consists of positive definite forms. Thus, $D_{A} \subset \mathrm{NS}(A) \otimes \mathbb{R}$ is the Siegel half-space consisting of symmetric bilinear forms on $\Lambda \otimes \mathbb{C}$ with positive definite imaginary part.

According to [12] (see also [27, Section 6.5]), one can view the abelian variety $B$ associated with an element $\omega=\omega_{A} \in D_{A} \simeq \mathfrak{H}_{n}$ as a mirror dual to $\left(A, \omega_{A}\right)$. More precisely, let us set

$$
\Gamma_{B}=\Lambda^{*} \oplus \Lambda, \quad B=\Gamma_{B} \otimes \mathbb{R} / \Gamma_{B}
$$

and define the complex structure on $\Gamma_{B} \otimes \mathbb{R}$ via the isomorphism

$$
\kappa_{\omega}: \Gamma_{B} \otimes \mathbb{R} \rightarrow \Lambda^{*} \otimes \mathbb{C}:\left(\lambda^{*}, \lambda\right) \mapsto \lambda^{*}-\omega(\lambda),
$$

where we view $\omega$ as an element of $\operatorname{Hom}\left(\Lambda, \Lambda^{*} \otimes \mathbb{C}\right)^{+}$. Note that there is an isomorphism $B \simeq \Lambda^{*} \otimes \mathbb{C} /\left(\Lambda^{*}+\omega \Lambda\right)$. (However, the corresponding identification of $H_{1}(B, \mathbb{Z})$ with $\Gamma_{B}$ differs from the original one by the sign on the summand $\Lambda \subset \Gamma_{B}$.) We have a natural principal polarization $\phi_{0}: B \Im \hat{B}$ given on homology lattices by

$$
\Gamma_{B} \rightarrow \Gamma_{B}^{*}:\left(\lambda_{0}^{*}, \lambda_{0}\right) \mapsto\left(\lambda^{*}, \lambda\right) \mapsto \lambda^{*}\left(\lambda_{0}\right)-\lambda_{0}^{*}(\lambda) .
$$

Similarly, the natural isomorphism $\Lambda^{*} \oplus \Lambda^{*} \simeq \Gamma_{\hat{A}} \simeq \Gamma_{A}^{*}$ corresponds to the pairing

$$
\left(\Lambda^{*} \oplus \Lambda^{*}\right) \times \Gamma_{A} \rightarrow \mathbb{Z}:\left(\left(\lambda_{1}^{*}, \lambda_{2}^{*}\right),\left(\lambda_{1}, \lambda_{2}\right)\right) \mapsto \lambda_{1}^{*}\left(\lambda_{2}\right)-\lambda_{2}^{*}\left(\lambda_{1}\right) .
$$

Let us define an isomorphism of orthogonal lattices

$$
\gamma: \Gamma_{A} \oplus \Gamma_{\hat{A}} \rightarrow \Gamma_{B} \oplus \Gamma_{B} \simeq \Gamma_{B} \oplus \Gamma_{\hat{B}}:\left(\lambda_{1}, \lambda_{2}, \lambda_{1}^{*}, \lambda_{2}^{*}\right) \mapsto\left(\lambda_{2}^{*}, \lambda_{2}, \lambda_{1}^{*}, \lambda_{1}\right)
$$




\section{PROPOSITION 3.5.1}

The isomorphism $\gamma$ induces a mirror duality in the sense of [12, Section 9] between the pairs $\left(A, \omega_{A}\right)$ and $\left(B, \omega_{B}\right)$ for $\omega_{B}=\tau \cdot \phi_{0}$, where $\phi_{0} \in \operatorname{Hom}(B, \hat{B})^{+}$ is the principal polarization defined above.

\section{Proof}

By definition, we have to check that the operator of complex structure on $\left(\Gamma_{B} \oplus\right.$ $\left.\Gamma_{B}\right) \otimes \mathbb{R}$ corresponds under $\gamma$ to

$$
I_{\omega_{A}}=\left(\begin{array}{cc}
\alpha^{-1} \beta & -\alpha^{-1} \\
\alpha+\beta \alpha^{-1} \beta & -\beta \alpha^{-1}
\end{array}\right) \in \mathbf{U}_{A}(\mathbb{R}),
$$

where $\omega_{A}=i \alpha+\beta$, and we view $\mathbf{U}_{A}(\mathbb{R})$ as a subgroup in automorphisms of $\left(\Gamma_{A} \oplus \Gamma_{\hat{A}}\right) \otimes \mathbb{R}$, and similarly that the complex structure on $\left(\Gamma_{A} \oplus \Gamma_{\hat{A}}\right) \otimes \mathbb{R}$ corresponds to $I_{\omega_{B}}$. Both facts are checked by a straightforward computation (cf. [12, Proposition 9.6.1]).

Recall that the variety $\mathbf{L} \mathbf{G}_{A}=\mathbf{L} \mathbf{G}_{E^{n}}$ is naturally identified with the Lagrangian Grassmannian associated with the symplectic lattice $\Lambda^{*} \oplus \Lambda$. Thus, a Lagrangian subvariety $L \subset A \times \hat{A}$, viewed as a point of $\mathbf{L G}(\mathbb{Q})$, corresponds to a Lagrangian $\mathbb{Z}$-submodule $\Pi(L) \subset \Lambda^{*} \oplus \Lambda=\Gamma_{B}$, so that $\Gamma_{L}=H_{1}(L, \mathbb{Z}) \simeq \Pi(L) \oplus \Pi(L) \subset$ $\Gamma_{A} \oplus \Gamma_{\hat{A}}$. Hence, from (3.2.3) we get

$$
\delta(L)(\omega)=\operatorname{det}\left(\left.\kappa_{\omega}\right|_{\Pi(L)}\right)^{2} \bmod \mathbb{R}_{>0},
$$

where we view $\left.\kappa_{\omega}\right|_{\Pi(L)}$ as an element in $\operatorname{Hom}\left(\Pi(L), \Lambda^{*}\right) \otimes \mathbb{C}$ and define $\operatorname{det}^{2}$ using some bases in $\Pi(L)$ and $\Lambda^{*}$.

Similarly, a point $L$ of $\mathbf{L G}_{A}(\mathbb{R})$ corresponds to a real Lagrangian subspace $\Pi_{\mathbb{R}}(L) \subset \Gamma_{B} \otimes \mathbb{R}$, and the formula (3.5.3) still holds (with $\Pi_{\mathbb{R}}(L)$ instead of $\Pi(L)$ ). Recall that we have a double covering $\mathbf{L G}^{\text {spin }}(A, \mathbb{R}) \rightarrow \mathbf{L G}(\mathbb{R})$ consisting of pairs $(L, \varphi) \in \mathbf{L G}_{A}(\mathbb{R}) \times \mathcal{O}\left(D_{A}\right) / \mathbb{R}_{>0}$ such that $\varphi^{2}=\delta(L)$, so that the group $\operatorname{Spin}(\mathbb{R})$ acts on $\mathbf{L} \mathbf{G}^{\text {spin }}(A, \mathbb{R})$ (see Definition 3.2.4). In our case there is a splitting $\mathbf{U}(\mathbb{R}) \rightarrow$ $\operatorname{Spin}(\mathbb{R})$ (see Remark 2.3.8.1), so we have an action of $\mathbf{U}(\mathbb{R})$ on $\mathbf{L G}^{\text {spin }}(A, \mathbb{R})$ given by

$$
g \cdot(L, \varphi)=\left(g L, \varphi^{\prime}\right), \quad \text { where } \varphi^{\prime}(g(\omega))=\varphi(\omega) \cdot \operatorname{det}(a+b \omega)^{-1} .
$$

We claim that $\mathbf{L} \mathbf{G}^{\text {spin }}(A, \mathbb{R}) \rightarrow \mathbf{L G}_{A}(\mathbb{R})$ is in fact the natural double covering corresponding to a choice of orientation on a Lagrangian subspace in $\Gamma_{B} \otimes \mathbb{R}$. Indeed, let us fix an orientation $\epsilon \in \bigwedge^{n}(\Lambda)$. Then a choice of a square root $\varphi=$ $\sqrt{\delta(L)} \in \mathcal{O}^{*}\left(D_{A}\right) / \mathbb{R}_{>0}$ for $L \in \mathbf{L G}_{A}(\mathbb{R})$ induces an orientation on $\Pi_{\mathbb{R}}(L) \subset \Gamma_{B} \otimes$ $\mathbb{R}$ as follows. By formula (3.5.3), for each $\omega$ the nonzero element

$$
\varphi(\omega)^{-1} \cdot \operatorname{det}\left(\left.\kappa_{\omega}\right|_{\Pi_{\mathbb{R}}(L)}\right) \in \bigwedge^{n}\left(\Lambda^{*}\right) \otimes \bigwedge^{n}\left(\Pi_{\mathbb{R}}(L)\right)^{-1} \otimes_{\mathbb{R}} \mathbb{C}
$$

depending continuously on $\omega$, belongs to the $\mathbb{R}$-subspace $\bigwedge^{n}\left(\Lambda^{*}\right) \otimes \bigwedge^{n}\left(\Pi_{\mathbb{R}}(L)\right)^{-1}$. Thus, we get an isomorphism

$$
\bigwedge^{n}\left(\Pi_{\mathbb{R}}(L)\right) \simeq \bigwedge^{n}(\Lambda)^{-1} \otimes \mathbb{R}
$$


and we define the orientation $\mu_{\varphi, \epsilon} \in \bigwedge^{n}\left(\Pi_{\mathbb{R}}(L)\right)$ so that it corresponds to $\epsilon^{-1}$ under this isomorphism, that is,

$$
\varphi(\omega)^{-1} \cdot \operatorname{det}\left(\left.\kappa_{\omega}\right|_{\Pi_{\mathbb{R}}(L)}\right) \cdot \mu_{\varphi, \epsilon}=\epsilon^{-1} .
$$

Let us associate with $L \in \mathbf{L G}_{A}(\mathbb{Q})$ the real subtorus in $B$ by setting

$$
T_{L}=\Pi(L) \otimes \mathbb{R} / \Pi(L) \subset \Gamma_{B} \otimes \mathbb{R} / \Gamma_{B}=B .
$$

Note that $T_{L}$ is Lagrangian with respect to the translation-invariant symplectic structure on $B$ corresponding to the standard symplectic structure on $\Gamma_{B}=\Lambda^{*} \oplus$ $\Lambda$ (i.e., this symplectic structure on $B$ comes from the principal polarization $\phi_{0}$ ). As we have shown above, a lifting of $L$ to a point $(L, \varphi) \in \mathbf{L} \mathbf{G}^{\operatorname{spin}}(A, Q)$ gives rise to an orientation of $T_{L}$.

Since $\mathbf{L} \mathbf{G}^{\operatorname{spin}}(A, \mathbb{Q})=\widetilde{\mathbf{L} \mathbf{G}_{A}(\mathbb{Q})} / 2 \mathbb{Z}$, the map (3.2.4) induces a map

$$
\overline{\mathrm{SH}}^{\mathrm{LI}} \rightarrow \mathbf{L} \mathbf{G}^{\mathrm{spin}}(A, \mathbb{Q}) \text {. }
$$

By Lemma 3.2.5, the composition

$$
\mathrm{NS}(A) \otimes \mathbb{Q} \rightarrow \overline{\mathrm{SH}}^{\mathrm{LI}} \rightarrow \mathbf{L G}^{\mathrm{spin}}(A, \mathbb{Q}): \phi \mapsto V_{\phi} \mapsto(\Gamma(\phi), \varphi)
$$

corresponds to the choice of the square root $\varphi(\omega)=\operatorname{det}(\phi-\omega)$, where we use dual bases of $\Lambda$ and $\Lambda^{*}$ to compute the determinant (see also Example 3.2.6). The corresponding orientation on $T_{\Gamma(\phi)}$ is induced by the isomorphism $\Pi(\Gamma(\phi)) \otimes \mathbb{R} \simeq$ $\Lambda \otimes \mathbb{R}$ and the orientation $\epsilon$ of $\Lambda \otimes \mathbb{R}$.

Let $\Omega_{\omega, \epsilon}$ denote the holomorphic volume form on $B$ defined by

$$
\Omega_{\omega, \epsilon}=\kappa_{\omega}^{*}(\epsilon),
$$

where we view $\epsilon \in \Lambda^{n}(\Lambda) \subset \Lambda^{n}(\Lambda) \otimes \mathbb{C}$ as an $n$-form on $\Lambda^{*} \otimes \mathbb{C}$ and use the isomorphism (3.5.1).

\section{THEOREM 3.5.2}

For an endosimple LI object $F \in D^{b}(A)$ one has

$$
\chi(\ell(\omega),[F])=\int_{\left[T_{L}\right]} \Omega_{\omega, \epsilon},
$$

where $L=L_{F}$ and $T_{L}$ is equipped with the orientation $\mu_{\varphi_{F}, \epsilon}$ coming from the point $\left(L_{F}, \varphi_{F}\right) \in \mathbf{L G}^{\operatorname{spin}}(A, \mathbb{Q})$ associated with $F$.

\section{Proof}

Note that shifting $F$ by [1] changes the orientation of $T_{L}$, so the assertions for $F$ and $F[n]$ are equivalent.

First, let us prove (3.5.4) in the case when $L_{F}$ is transversal to $\{0\} \times \hat{A}$, that is, when $F=V_{\phi}$ is the semihomogeneous bundle corresponding to $\phi \in$ $\operatorname{Hom}(A, \hat{A})^{+} \otimes \mathbb{Q} \simeq \operatorname{Hom}\left(\Lambda, \Lambda^{*}\right)^{+} \otimes \mathbb{Q}$ (and $\left.L_{F}=\Gamma(\phi) \subset A \times \hat{A}\right)$. Recall that $\operatorname{rk} V_{\phi}=\operatorname{deg}\left(L_{F} \rightarrow A\right)^{1 / 2}$ (see (2.1.9)). For $K=\mathbb{Q}$ or $\mathbb{R}$ let $\Gamma_{K}(\phi) \subset\left(\Lambda^{*} \oplus \Lambda\right) \otimes K$ be the graph of $\phi$ viewed as a map of $K$-vector spaces (i.e., $\Gamma_{K}(\phi)=H_{1}\left(L_{F}, K\right)$ ), 
and set

$$
\Gamma_{\mathbb{Z}}(\phi):=\Gamma_{\mathbb{Q}}(\phi) \cap\left(\Lambda^{*} \oplus \Lambda\right),
$$

so that $T_{L}=\Gamma_{\mathbb{R}}(\phi) / \Gamma_{\mathbb{Z}}(\phi)$. We also denote by $i_{\phi}: \Gamma_{\mathbb{Z}}(\phi) \rightarrow \Lambda^{*} \oplus \Lambda$ the natural embedding. The orientation on $T_{L}$ is induced by the natural isomorphism $\Gamma_{\mathbb{R}}(\phi) \simeq$ $\Lambda \otimes \mathbb{R}$ and by the orientation of $\Lambda \otimes \mathbb{R}$ given by $\epsilon$. The cycle $\left[T_{L}\right]$ in $H_{n}(A) \simeq$ $\Lambda^{n}\left(\Lambda^{*} \oplus \Lambda\right)$ is the image of the positive generator $\mu \in \Lambda^{n}\left(\Gamma_{\mathbb{Z}}(\phi)\right)$ under the map

$$
\bigwedge^{n}\left(i_{\phi}\right): \bigwedge^{n}\left(\Gamma_{\mathbb{Z}}(\phi)\right) \rightarrow \bigwedge^{n}\left(\Lambda^{*} \oplus \Lambda\right)
$$

Note also that the integration map

$$
H_{n}(A) \rightarrow \mathbb{C}: \gamma \mapsto \int_{\gamma} \Omega_{\omega, \epsilon}
$$

is identified with

$$
\bigwedge^{n}\left(\kappa_{\omega}\right): \bigwedge^{n}\left(\Lambda^{*} \oplus \Lambda\right) \rightarrow \bigwedge^{n}\left(\Lambda^{*}\right) \otimes \mathbb{C} \simeq \mathbb{C}
$$

where the last isomorphism is given by $\epsilon$. Hence, $\int_{\left[T_{L}\right]} \Omega_{\omega, \epsilon}=\delta(\mu) \cdot \epsilon$, where $\delta \in \bigwedge^{n}\left(\Lambda^{*}\right) \otimes \bigwedge^{n}\left(\Gamma_{\mathbb{Z}}(\phi)\right)^{-1} \otimes \mathbb{C}$ is the determinant of the composition

$$
\Gamma_{\mathbb{Z}}(\phi) \stackrel{i_{\phi}}{\longrightarrow} \Lambda^{*} \oplus \Lambda \stackrel{\kappa_{\omega}}{\longrightarrow} \Lambda^{*} .
$$

The projection $p_{2}: \Gamma_{\mathbb{Z}}(\phi) \rightarrow \Lambda$ is an embedding of index $\operatorname{deg}\left(L_{F} \rightarrow A\right)^{1 / 2}$, so the commutative diagram

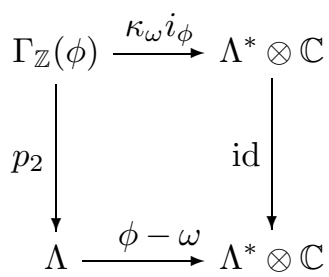

implies that

$$
\delta(\mu) \cdot \epsilon=\operatorname{det}(\phi-\omega) \cdot \operatorname{deg}\left(L_{F} \rightarrow A\right)^{1 / 2}=\chi(\ell(\omega), \ell(\phi)) \cdot \operatorname{rk}(F)=\chi(\ell(\omega),[F]),
$$

where the last equality follows from Lemma 2.5.2.

Next, we will check that (3.5.4) is compatible with the action of the group $\mathbf{U}(\mathbb{Z})$ on $[F], \omega$, and $B$, where we use the natural symplectic action of $\mathbf{U}(\mathbb{Z})$ on $\Gamma_{B}=\Lambda \oplus \Lambda^{*}$ and the splitting $\mathbf{U}(\mathbb{Z}) \rightarrow \mathbf{U}(\mathbb{Z})^{\text {spin }}$ of the spin covering (see Remark 2.3.8.1). Namely, for $g=\left(\begin{array}{ll}a & b \\ c & d\end{array}\right) \in \mathbf{U}(\mathbb{Z})$ the relation

$$
\left(\begin{array}{ll}
-\omega & \operatorname{id}_{\Lambda^{*}}
\end{array}\right) \cdot g^{-1}=(a+b \omega)^{*} \cdot\left(-g(\omega) \quad \operatorname{id}_{\Lambda^{*}}\right)
$$

leads to a commutative diagram

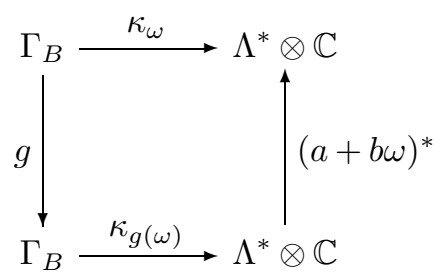


Hence, we have

$$
g^{*} \Omega_{g(\omega), \epsilon}=\operatorname{det}(a+b \omega)^{-1} \cdot \Omega_{\omega, \epsilon}
$$

which implies that

$$
\int_{g\left[T_{L}\right]} \Omega_{g(\omega), \epsilon}=\operatorname{det}(a+b \omega)^{-1} \cdot \int_{\left[T_{L}\right]} \Omega_{\omega, \epsilon} .
$$

Also, the diagram (3.5.5) gives the equation

$$
\left.\kappa_{\omega}\right|_{\Pi(L)}=\left.\left.(a+b \omega)^{*} \circ \kappa_{g(\omega)}\right|_{\Pi(g L)} \circ g\right|_{\Pi(L)}
$$

in $\operatorname{Hom}\left(\Pi(L), \Lambda^{*}\right) \otimes \mathbb{C}$. Since $g \cdot(L, \varphi)=\left(g L, \varphi^{\prime}\right)$, where $\varphi^{\prime}(g(\omega))=\varphi(\omega) \operatorname{det}(a+$ $b \omega)^{-1}$, passing to determinants in (3.5.6) we obtain that the orientation $\mu_{\varphi^{\prime}, \epsilon}$ of $\Pi(g L) \otimes \mathbb{R}$ corresponds to $\mu_{\varphi, \epsilon}$ under the isomorphism $\Pi(L) \rightarrow \Pi(g L)$ given by $g$. Hence, the class $g\left[T_{L}\right]$ is exactly the fundamental class of $T_{g L}$ associated with the orientation coming from $g[F]$. On the other hand, by Corollary 2.5.6,

$$
\chi(\ell(\omega),[F])=\chi(\ell(g(\omega)), g[F]),
$$

since for $g \in \mathbf{U}(\mathbb{Z})$ the operator $\hat{\rho}(g)$ is simply the map induced by any autoequivalence of $D^{b}(A)$ compatible with the canonical lifting of $g$ to $\mathbf{U}(\mathbb{Z})^{\text {spin }}$.

Finally, applying Proposition 1.4 .1 and using the $\mathbf{U}(\mathbb{Z})$-invariance, we see that the general case of (3.5.4) follows from the case when $L_{F}$ is transversal to $\{0\} \times \hat{A}$ considered above.

\section{REMARK 3.5.3}

Note that since $L_{F}$ is equipped with the lifting $\widetilde{L}_{F}$ to the universal covering of the Lagrangian Grassmannian (see Example 3.2.6), the Lagrangian torus $T_{L}$ has a structure of a graded Lagrangian (see [33]). The corresponding choice of a phase of $\int_{T_{L}}$ obtained from Theorems 3.3.2 and 3.5.2 comes from Kontsevich's description of a grading on a Lagrangian (see [33, Example 2.9]).

\section{Quasistandard $t$-structures and Fourier-Mukai partners}

\subsection{Quasistandard $t$-structures}

The $\mathbb{Z}$-covering $\widehat{\mathbf{L G}(\mathbb{Q})} \rightarrow \mathbf{L G}(\mathbb{Q})$ appears also naturally when considering $t$ structures. Let $\mathcal{T}(A)$ be the set of $\mathbf{H}$-invariant $t$-structures on $D^{b}(A)$. We identify $\mathcal{T}(A)$ with the set of cores of such $t$-structures, so we view elements of $\mathcal{T}(A)$ as abelian subcategories $\mathcal{A} \subset D^{b}(A)$.

THEOREM 4.1.1

(i) There is a natural $\widehat{\mathbf{U}(\mathbb{Q})}$-equivariant embedding

$$
\widetilde{\mathbf{L G}(\mathbb{Q})} \rightarrow \mathcal{T}(A): \widetilde{L} \mapsto \mathcal{A}_{\widetilde{L}},
$$

which is uniquely characterized by the condition

$$
\mathcal{A}_{\left(0: \phi_{0}\right), 0}=\operatorname{Coh}(A) \text {. }
$$


Thus, the LI functor $\Phi_{\widetilde{g}}: D^{b}(A) \rightarrow D^{b}(A)$ corresponding to $\widetilde{g} \in \widetilde{\mathbf{U}(\mathbb{Q})}$ (defined up to $\mathbf{H}$; see Section 2.1) satisfies

$$
\Phi_{\widetilde{g}}\left(\mathcal{A}_{\widetilde{L}}\right) \subset \mathcal{A}_{\widetilde{g} \widetilde{L}}
$$

(ii) For an $L I$ object $F$ and $\widetilde{L} \in \widetilde{\mathbf{L G}(\mathbb{Q})}$ one has $F\left[-i\left(\widetilde{L}_{F}, \widetilde{L}\right)\right] \in \mathcal{A}_{\widetilde{L}}$, where $i(\cdot, \cdot) \in \mathbb{Z}$ is the unique $\widetilde{\mathbf{U}(\mathbb{Q})}$-equivariant function on $\widehat{\mathbf{L G}(\mathbb{Q})} \times \widehat{\mathbf{L G}(\mathbb{Q})}$ such that for $\phi_{1}, \phi_{2} \in \mathrm{NS}(A) \otimes \mathbb{Q}$ one has

$$
i\left(\widetilde{L}_{V_{\phi_{1}}}, \widetilde{L}_{V_{\phi_{2}}}\right)=i\left(\phi_{2}-\phi_{1}\right),
$$

provided $\phi_{2}-\phi_{1}$ is nondegenerate. (Recall that $\widetilde{L}_{V_{\phi}}$ is given by (3.2.5).)

\section{Proof}

(i) Recall that the action of $\widetilde{\mathbf{U}(\mathbb{Q})}$ on $\widetilde{\mathbf{L G}(\mathbb{Q})}$ is transitive, and the stabilizer subgroup of the point $\left(\left(0: \phi_{0}\right), 0\right)$ is $\mathbf{P}^{-}(\mathbb{Q})$, lifted to $\widetilde{\mathbf{U}(\mathbb{Q})}$ as described in Corollary 2.2.2. Thus, it suffices to check that $\mathbf{P}^{-}(\mathbb{Q})$ preserves the standard $t$-structure. But this immediately follows from the description of the functors corresponding to elements of $\mathbf{P}^{-}(\mathbb{Q})$ (see Proposition 2.2.1).

(ii) The fact that every LI sheaf is cohomologically pure with respect to each $t$-structure constructed in (i) follows from Theorem 2.4.1. The uniqueness of the $\widetilde{\mathbf{U}(\mathbb{Q})}$-equivariant index function $i(\cdot, \cdot)$ follows from Proposition 1.4.1. It remains to find the number $i=i\left(\phi_{1}, \phi_{2}\right)$ such that

$$
V_{\phi_{1}}[-i] \in \mathcal{A}_{\widetilde{L}_{V_{\phi_{2}}}} .
$$

Let $g=\left(\begin{array}{cc}1 & \phi_{2}^{-1} \\ 0 & 1\end{array}\right)$. Then by formula (2.4.1), we have

$$
\Phi_{g}\left(\mathcal{O}_{x}\right)=V_{\phi_{2}} \bmod \mathbb{N}^{*} .
$$

(There is no shift in this case since the kernel $S(g)$ is a vector bundle.) It follows that

$$
\Phi_{g}(\operatorname{Coh}(A)) \subset \mathcal{A}_{\widetilde{L}_{V_{\phi_{2}}}} .
$$

Note that $\Gamma\left(\phi_{1}\right)=g(\Gamma(\phi))$, where

$$
\phi=\phi_{1}\left(1-\phi_{2}^{-1} \phi_{1}\right)^{-1} .
$$

Hence, using (2.4.1) and (2.4.3) we obtain

$$
\Phi_{g}\left(V_{\phi}\right)=V_{\phi_{1}}\left[-i\left(\phi_{2}+\phi\right)\right] \bmod \mathbb{N}^{*},
$$

so denoting $\phi_{3}=1-\phi_{2}^{-1} \phi_{1}$ we obtain

$$
i=i\left(\phi_{2}+\phi_{1} \phi_{3}^{-1}\right)=i\left(\phi_{3}\left(\phi_{2} \phi_{3}+\phi_{1}\right)\right)=i\left(\phi_{3} \phi_{2}\right)=i\left(\phi_{2}-\phi_{1}\right)
$$

as claimed.

\section{DEFINITION 4.1.2}

We will refer to $t$-structures on $D^{b}(A)$ constructed in the above theorem as quasistandard t-structures. 


\section{PROPOSITION 4.1.3}

Let $A$ and $B$ be abelian varieties, and let $\eta: X_{A} \rightarrow X_{B}$ be a symplectic isomorphism in $\mathcal{A} \mathrm{b}_{\mathbb{Q}}$ (i.e., up to isogeny). Then the map $\eta_{*}: \mathbf{L G}_{A}(\mathbb{Q}) \rightarrow \mathbf{L G}_{B}(\mathbb{Q})$ induced by $\eta$ extends to a $\mathbb{Z}$-equivariant map

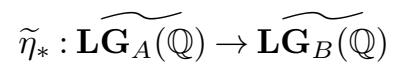

which is compatible with the quasistandard t-structures; that is, for every $\widetilde{L} \in$ $\widetilde{\mathbf{L} \mathbf{G}_{A}(\mathbb{Q})}$ the LI functor $\Phi_{\eta}$ associated with $\eta$ (defined up to $\mathbf{H}$ ) satisfies

$$
\Phi_{\eta}\left(\mathcal{A}_{\widetilde{L}}\right) \subset \mathcal{A}_{\widetilde{\eta}_{*} \widetilde{L}}
$$

Proof

Note that $B$ is isogenous to $A$; that is, there exists an isomorphism $f: A \rightarrow B$ in $\mathcal{A} \mathrm{b}_{\mathbb{Q}}$. Let $\eta_{0}: X_{A} \rightarrow X_{B}$ be the induced symplectic isomorphism in $\mathcal{A} \mathrm{b}_{\mathbb{Q}}$. We also have natural compatible isomorphisms induced by $f$ :

$$
\begin{aligned}
& \mathbf{U}_{X_{A}} \rightarrow \mathbf{U}_{X_{B}}, \quad \widetilde{\mathbf{U}_{X_{A}}(\mathbb{Q})} \rightarrow \widetilde{\mathbf{U}_{X_{B}}(\mathbb{Q})}, \\
& \eta_{0 *}: \mathbf{L G}_{A}(\mathbb{Q}) \rightarrow \mathbf{L G}_{B}(\mathbb{Q}), \quad \widetilde{\eta}_{0 *}: \widetilde{\mathbf{L} \mathbf{G}_{A}(\mathbb{Q})} \rightarrow \widetilde{\mathbf{L} \mathbf{G}_{B}(\mathbb{Q})} .
\end{aligned}
$$

Furthermore, it is easy to see that the $t$-exactness (4.1.1) holds for $\widetilde{\eta}_{0 *}$ and the functor $\Phi_{\eta_{0}}$ which is the composition of the pullback and the pushforward under isogenies. (This is proved similarly to Proposition 2.2.1(ii).) Now let $g_{\eta} \in \mathbf{U}(\mathbb{Q}$ ) be the unique element such that

$$
\eta=\eta_{0} \circ g_{\eta}
$$

Choose any element $\widetilde{g}_{\eta} \in \widetilde{\mathbf{U}(\mathbb{Q})}$ over $g_{\eta}$, and define

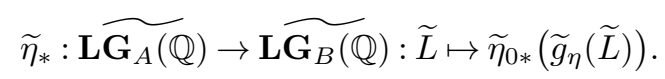

By Theorem 4.1.1(i), the required assertion follows for the functor $\Phi_{\eta_{0}} \circ \Phi_{\widetilde{g}_{\eta}}$. By [31, Theorem 3.2.11], its $\mathbf{H}$-equivalence class differs from $\Phi_{\eta}[n]$ by an action of $\mathbb{N}^{*}$. (One has to use also [31, Proposition 2.4.7(ii)] as in the proof of $[31$,

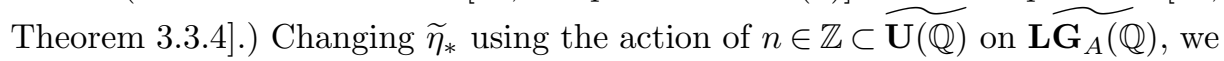
get the required compatibility (4.1.1).

\section{REMARKS 4.1 .4}

(1) The quasistandard $t$-structure associated with $\widetilde{L}_{F} \in \widetilde{\mathbf{L G}(\mathbb{Q})}$ has a simple characterization in terms of the LI object $F$ (defined up to H-equivalence). Namely, the corresponding subcategory $D^{\leq 0} \subset D^{b}(A)$ consists of all $X \in D^{b}(A)$ such that $\operatorname{Hom}^{i}\left(X, T_{x, \xi}(F)\right)=0$ for $i<0$ and all $(x, \xi) \in A \times \hat{A}$. Indeed, by using $\widetilde{\mathbf{U}(\mathbb{Q})}$ action this reduces to the characterization of the standard subcategory $D^{\leq 0}$ by the above condition, where $F$ is a nonzero torsion sheaf.

(2) In the case of an elliptic curve all the quasistandard $t$-structures are obtained from the standard one by tilting (up to a shift). More precisely, let $P(\cdot)$ be the slicing associated with the standard stability on $D^{b}(E)$ for an elliptic curve $E$, so that $P((0,1])=\operatorname{Coh}(E)$ (see Example 3.3.5). Then the quasistandard 
$t$-structure associated with $\phi \in \mathrm{NS}(E, \mathbb{Q}) \simeq \mathbb{Q}\left(\right.$ lifted to $\widetilde{\mathbf{L} \mathbf{G}_{E}(\mathbb{Q})}$ by $\left.(3.2 .6)\right)$ is $P((\operatorname{Arg}(i-\phi)) / \pi-1,(\operatorname{Arg}(i-\phi)) / \pi])$. Note that this construction extends to irrational numbers $\phi \in \mathbb{R}$ and for $k=\mathbb{C}$ the corresponding hearts are equivalent to the categories of holomorphic bundles on noncommutative 2-tori (see [28], $[32])$. We conjecture that this connection between quasistandard $t$-structures and noncommutative tori extends to the higher-dimensional case. (The corresponding equivalence of derived categories is established in [4].) Namely, to every point of $\widetilde{\mathbf{L} \mathbf{G}_{A}(\mathbb{R})} \backslash \widetilde{\mathbf{L} \mathbf{G}_{A}(\mathbb{Q})}$ there should correspond a $t$-structure on $D^{b}(A)$ (in a way compatible with the action of $\widetilde{\mathbf{U}(\mathbb{Q})}$ ) whose heart is equivalent to the category of holomorphic bundles on the corresponding noncommutative torus.

\subsection{Fourier-Mukai partners}

Recall that the set of Fourier-Mukai partners (FM partners for short) of a smooth projective variety $X$ is defined as

$$
\operatorname{FM}(X)=\left\{Y \text { smooth projective } \mid D^{b}(Y) \simeq D^{b}(X)\right\} / \text { isomorphism. }
$$

For an abelian variety $A$ we can also define the subset $\operatorname{FM}^{a b}(A) \subset \operatorname{FM}(A)$ by considering only FM partners among abelian varieties. In characteristic zero it is known that $\mathrm{FM}^{a b}(A)=\mathrm{FM}(A)$ (see [14]).

Recall that if $B$ is an FM partner of $A$, then any equivalence $D^{b}(A) \simeq D^{b}(B)$ is given by the LI kernel associated with a Lagrangian correspondence $(L(\eta), \alpha)$ extending a symplectic isomorphism $\eta: X_{A} \simeq X_{B}$ (see Section 2.1). The $\mathbf{U}(\mathbb{Z})$ orbit of the Lagrangian $\left(\eta_{*}\right)^{-1}(0 \times \hat{B}) \in \mathbf{L G}_{A}(\mathbb{Q})$ does not depend on a choice of an equivalence $D^{b}(A) \simeq D^{b}(B)$.

\section{PROPOSITION 4.2.1}

The above construction gives an embedding

$$
\mathrm{FM}^{a b}(A) \hookrightarrow \mathbf{L G}_{A}(\mathbb{Q}) / \mathbf{U}(\mathbb{Z}) .
$$

The image consists of orbits of Lagrangian subvarieties $L \subset X_{A}$ for which there exists a Lagrangian subvariety $L^{\prime} \subset X_{A}$ such that $L \cap L^{\prime}=0$.

Proof

The first assertion is immediate since the Lagrangian subvariety $\left(\eta_{*}\right)^{-1}(0 \times \hat{B}) \subset$ $X_{A}$ corresponding to $B$ is isomorphic to $\hat{B}$. For the second we observe that if we have a Lagrangian $L^{\prime} \subset X_{A}$ such that $L \cap L^{\prime}=0$, then we get an isomorphism $L \times L^{\prime} \rightarrow X_{A}$ and also $L^{\prime} \simeq X_{A} / L \simeq \hat{L}$, which leads to a symplectic isomorphism $L \times \hat{L} \simeq X_{A}$, so that $B=\hat{L}$ is an FM partner of $A$.

\section{REMARK 4.2.2}

The set $\mathbf{L G}_{A}(\mathbb{Q}) / \mathbf{U}(\mathbb{Z})=\mathbf{U}(\mathbb{Z}) \backslash \mathbf{U}(\mathbb{Q}) / \mathbf{P}^{-}(\mathbb{Q})$ is known to be finite (see $[11$, Theorem 6$]$ ). Note that this set is also in bijection with the set of endosimple LI objects in $D^{b}(A)$ up to the action of exact autoequivalences of $D^{b}(A)$ (as follows from Proposition 2.1.2). 
Here is an example of a situation when the embedding of Proposition 4.2.1 is a bijection.

\section{PROPOSITION 4.2.3}

Assume that $A$ is principally polarized, and assume that $\operatorname{End}(A)=R$ is the ring of integers in a totally real number field $F$ (so the Rosati involution on $F$ is trivial). Then the map (4.2.1) is a bijection, and

$$
\left|\mathrm{FM}^{a b}(A)\right|=\left|\mathbf{L} \mathbf{G}_{A}(\mathbb{Q}) / \mathbf{U}(\mathbb{Z})\right|=h_{R},
$$

where $h_{R}$ is the class number of $R$.

Proof

First, we observe that in this case the set $\mathbf{L G}_{A}(\mathbb{Q})$ consists of all subvarieties in $X_{A}=A \times A$, isogenous to $A$. We claim that all such subvarieties $L \subset X_{A}$ are direct summands. Indeed, $L$ is an image of the morphism $A \rightarrow A^{2}$ associated with a pair $(a, b) \in R^{2} \backslash\{(0,0)\}$. Consider the exact sequence

$$
0 \rightarrow I^{\prime} \rightarrow R^{2} \rightarrow I \rightarrow 0
$$

where $I=(a, b) \subset R$. This sequence splits since $I$ is a projective $R$-module. Hence, there is a corresponding split exact sequence of abelian varieties

$$
0 \rightarrow A^{I} \rightarrow A^{2} \rightarrow A^{I^{\prime}} \rightarrow 0
$$

where we use the natural functor $M \rightarrow A^{M}$ from $R$-modules to commutative group schemes with $A^{M}(S)=\operatorname{Hom}_{R}\left(M, A(S)\right.$ ) (see [10]). Since $A^{I}$ is exactly the image of the map $(a, b): A \rightarrow A^{2}$, this proves our claim.

It remains to check that the orbits of $\mathrm{SL}_{2}(R)$ on the projective line $\mathbb{P}^{1}(F)$ are in bijection with the ideal class group $\mathrm{Cl}(R)$. We have a well-defined map

$$
\mathbb{P}^{1}(F) / \mathrm{SL}_{2}(R) \rightarrow \mathrm{Cl}(R)
$$

sending ( $a: b)$ with $a, b \in R$ to the class of the ideal $(a, b)$. This map is surjective since every nonzero ideal in $R$ is generated by two elements. To show injectivity suppose that pairs $(a: b)$ and $\left(a^{\prime}: b^{\prime}\right)$ define the same ideal class. Then upon rescaling we can assume that $(a, b)=\left(a^{\prime}, b^{\prime}\right)$. Now we have two surjective maps $R^{2} \rightarrow I=(a, b)$, one given by $(a, b)$ and another by $\left(a^{\prime}, b^{\prime}\right)$, and our assertion follows from Lemma 4.2.4 below.

LEMMA 4.2.4

For every nonzero ideal $I \subset R$ the action of $\mathrm{SL}_{2}(R)$ on surjective maps $R^{2} \rightarrow I$ is transitive.

\section{Proof}

Since $I$ is a projective $R$-module, for every surjective map $f: R^{2} \rightarrow I$ there exists an isomorphism

$$
\alpha: R^{2} \stackrel{\sim}{\longrightarrow} I^{\prime} \oplus I
$$


such that $f$ is the composition of $\alpha$ with the projection to $I$. Note that $\operatorname{det}(\alpha)$ induces an isomorphism of $R$ with $I^{\prime} \otimes_{R} I$, so we obtain an isomorphism $I^{\prime} \simeq I^{-1}$. Thus, we can view $\alpha$ as an isomorphism $R^{2} \rightarrow I^{-1} \oplus I$ such that $\operatorname{det}(\alpha)$ is the canonical isomorphism $R \rightarrow I^{-1} \otimes I$. If $g: R^{2} \rightarrow I$ is another surjective morphism and $\beta: R^{2} \stackrel{\sim}{\longrightarrow} I^{-1} \oplus I$ is the corresponding isomorphism, then $\gamma=\beta^{-1} \circ \alpha$ is an element of $\mathrm{SL}_{2}(R)$ such that $g \circ \gamma=f$.

\section{REMARK 4.2.5}

In general the embedding (4.2.1) is not a bijection as one can see already in the case of a nonprincipally polarized abelian variety with $\operatorname{End}(A)=\mathbb{Z}$ (cf. $[23$, Example 4.16]). The Lagrangians not in the image of this map correspond to categories of twisted sheaves equivalent to $D^{b}(A)$ (see [25]). Note that the set $\mathbf{L G}_{A}(\mathbb{Q})$ is a subset of vertices of the spherical building associated with the group $\mathbf{U}$, which is related to the boundary of the Baily-Borel compactification of the Siegel domain $D_{A}$. It would be interesting to see whether other elements of this building have an interpretation in terms of $D^{b}(A)$. Also, one can expect some relation between the quasistandard $t$-structures and the $t$-structures associated with stabilities coming from points of $D_{A}$ or $D_{A} \times \mathbb{C}$. In the case of K3-surfaces similar questions are studied in [15] and [13].

Acknowledgments. I am grateful to Tom Bridgeland for helpful discussions and to Maxim Kontsevich for a discussion of the picture in Section 3.5 involving mirror symmetry.

\section{References}

[1] D. Abramovich and A. Polishchuk, Sheaves of $t$-structures and valuative criteria for stable complexes, J. Reine Angew. Math. 590 (2006), 89-130. MR 2208130. DOI 10.1515/CRELLE.2006.005.

[2] A. Bayer, E. Macrì, and Y. Toda, Bridgeland stability conditions on threefolds I: Bogomolov-Gieseker type inequalities, J. Algebraic Geom. 23 (2014), 117-163.

[3] A. A. Beullinson, J. Bernstein, and P. Deligne, "Faisceaux pervers" in Analysis and Topology on Singular Spaces, I (Luminy, 1981), Astérisque 100, Soc. Math. France, Paris, 1982, 5-171. MR 0751966.

[4] J. Block, Duality and equivalence of module categories in noncommutative geometry II: Mukai duality for holomorphic noncommutative tori, preprint, arXiv:math/0604296v1 [math.QA].

[5] A. Borel, Linear Algebraic Groups, 2nd ed., Springer, New York, 1991. MR 1102012. DOI 10.1007/978-1-4612-0941-6.

[6] T. Bridgeland, Stability conditions on triangulated categories, Ann. of Math. (2) 166 (2007), 317-345. MR 2373143. DOI 10.4007/annals.2007.166.317.

[7] Stability conditions on K3 surfaces, Duke Math. J. 141 (2008), 241-291. MR 2376815. DOI 10.1215/S0012-7094-08-14122-5. 
[8] M. R. Douglas, "Dirichlet branes, homological mirror symmetry, and stability" in Proceedings of the International Congress of Mathematicians, Vol. III (Beijing, 2002), Higher Ed. Press, Beijing, 2002, 395-408. MR 1957548.

[9] W. Fulton, Intersection Theory, Ergeb. Math. Grenzgeb. (3) 2, Springer, Berlin, 1984. MR 0732620.

[10] J. Giraud, Remarque sur une formula de Shimura-Taniyama, Invent. Math. 5 (1968), 231-236. MR 0227172.

[11] R. Godement, "Domaines fondamentaux des groupes arithmétiques" in Séminaire Bourbaki, Vol. 8 (1962/1963), no. 257, Soc. Math. France, Paris, 1995, 201-225. MR 1611543.

[12] V. Golyshev, V. Lunts, and D. Orlov, Mirror symmetry for abelian varieties, J. Algebraic Geom. 10 (2001), 433-496. MR 1832329.

[13] H. Hartmann, Cusps of the Kähler moduli space and stability conditions on K3 surfaces, Math. Ann. 354 (2012), 1-42. MR 2957617.

DOI 10.1007/s00208-011-0719-3.

[14] D. Huybrechts and M. Nieper-Wisskirchen, Remarks on derived equivalences of Ricci-flat manifolds, Math. Z. 267 (2011), 939-963. MR 2776067.

DOI 10.1007/s00209-009-0655-z.

[15] S. Ma, Fourier-Mukai partners of a K3 surface and the cusps of its Kahler moduli, Internat. J. Math. 20 (2009), 727-750. MR 2541933.

DOI 10.1142/S0129167X09005510.

[16] A. Maciocia and D. Piyaratne, Fourier-Mukai transforms and Bridgeland stability conditions on Abelian threefolds, preprint, arXiv:1304.3887v3 [math.AG].

[17] Fourier-Mukai transforms and Bridgeland stability conditions on Abelian threefolds II, preprint, arXiv:1310.0299v1 [math.AG].

[18] E. Macrì, S. Mehrorta, and P. Stellari, Inducing stability conditions, J. Algebraic Geom. 18 (2009), 605-649.

[19] S. Mukai, Semi-homogeneous vector bundles on an Abelian variety, J. Math. Kyoto Univ. 18 (1978), 239-272. MR 0498572.

[20] Duality between $D(X)$ and $D(\hat{X})$ with its application to Picard sheaves, Nagoya Math. J. 81 (1981), 153-175. MR 0607081.

[21] _ "Abelian variety and spin representation" (in Japanese) in Hodge Theory and Algebraic Geometry (Sapporo, 1994), Hokkaido Univ., Sapporo, Japan; English translation in preprint, 1998.

[22] D. Mumford, Abelian Varieties, 2nd ed., Tata Inst. Fund. Res. Stud. Math. 5, Oxford Univ. Press, London, 1974. MR 0282985.

[23] D. O. Orlov, Derived categories of coherent sheaves on abelian varieties and equivalences between them (in Russian), Izv. Ross. Akad. Nauk Ser. Mat. 66 (2002), no. 3, 131-158; English translation in Izv. Math. 66 (2002), 569-594. MR 1921811. DOI 10.1070/IM2002v066n03ABEH000389. 
[24] A. Polishchuk, Biextensions, Weil representations on derived categories and theta-functions, Ph.D. dissertation, Harvard University, Cambridge, Mass., 1996. MR 2694692.

[25] _ Symplectic biextensions and a generalization of the Fourier-Mukai transform, Math. Res. Lett. 3 (1996), 813-828. MR 1426538.

DOI 10.4310/MRL.1996.v3.n6.a9.

[26] Analogue of Weil representation for abelian schemes, J. Reine Angew. Math. 543 (2002), 1-37. MR 1887877. DOI 10.1515/crll.2002.016.

[27] Abelian Varieties, Theta Functions and the Fourier Transform, Cambridge Tracts in Math. 153, Cambridge Univ. Press, Cambridge, 2003. MR 1987784. DOI 10.1017/CBO9780511546532.

[28]_Classification of holomorphic vector bundles on noncommutative two-tori, Doc. Math. 9 (2004), 163-181. MR 2054986.

[29] Constant families of t-structures on derived categories of coherent sheaves, Mosc. Math. J. 7 (2007), 109-134, 167. MR 2324559.

[30] Kernel algebras and generalized Fourier-Mukai transforms, J. Noncommut. Geom. 5 (2011), 153-251. MR 2784503. DOI 10.4171/JNCG/73.

[31] _ "Lagrangian-invariant sheaves and functors for abelian varieties" in Derived Categories in Algebraic Geometry, EMS Ser. Congr. Rep., Eur. Math. Soc., Zürich, 2012, 197-250. MR 3050705.

[32] A. Polishchuk and A. Schwarz, Categories of holomorphic vector bundles on noncommutative two-tori, Comm. Math. Phys. 236 (2003), 135-159. MR 1977884. DOI 10.1007/s00220-003-0813-9.

[33] P. Seidel, Graded Lagrangian submanifolds, Bull. Soc. Math. France 128 (2000), 103-149. MR 1765826.

[34] A. Weil, Sur certains groupes d'opérateurs unitaires, Acta Math. 111 (1964), 143-211. MR 0165033.

Department of Mathematics, University of Oregon, Eugene, Oregon 97405, USA;

apolish@uoregon.edu 